\title{
Sustainable LED Fluorescent Light Replacement Technology
}

\section{Final Report}

\author{
Prepared under: \\ NCMS Project No. 130190 and the \\ U.S. Department of Energy (DoE) \\ DE-FG26-08NT002239
}

September 2011

National Center for Manufacturing Sciences

3025 Boardwalk

Ann Arbor, Michigan 48108-3230 
(C2011 National Center for Manufacturing Sciences, Inc.

Neither NCMS, members of NCMS, nor any person acting on behalf of them:

- makes any warranty or representation, express or implied, with respect to the accuracy, completeness or usefulness of the information contained in this report, or that the use of any information, apparatus, method, or process disclosed in this report may not infringe privately owned rights, or

- assumes any liability with respect to the use of, or from damages resulting from the use of, any information, apparatus, method, or process disclosed in this report.

The views and conclusions contained herein are those of the authors and should not be interpreted as necessarily representing the official policies or endorsements, either expressed or implied, of the U.S. Government.

This material is based upon work supported by the Department of Energy (DoE) [National Nuclear Security Administration] under Award Number DE-FG26-08NT002239.

This report was prepared as an account of work sponsored by an agency of the United States Government. Neither the United States Government nor any agency thereof, nor any of their employees, makes any warranty, expressed or implied, or assumes any legal liability or responsibility for the accuracy, completeness, or usefulness of any information, apparatus, product, or process disclosed, or represents that its use would not infringe privately owned rights. Reference herein to any specific commercial product, process, or service by trade name, trademark, manufacturer, or otherwise does not necessarily constitute or imply its endorsement, recommendation, or favoring by the United States Government or any agency thereof. The views and opinions of authors expressed herein do not necessarily state or reflect those of the United States Government or any agency thereof. 


\section{Table of Contents}

Section

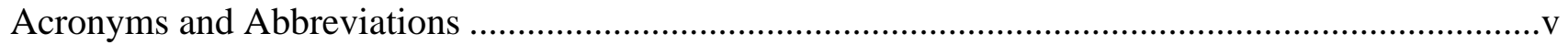

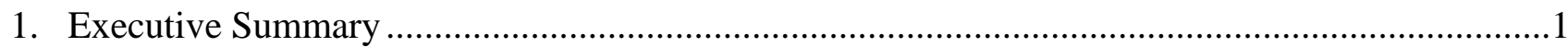

1.1 Project Partners .....................................................................................................

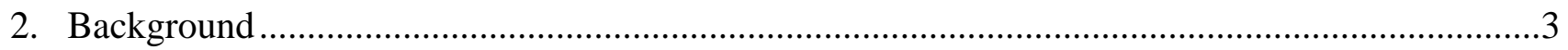

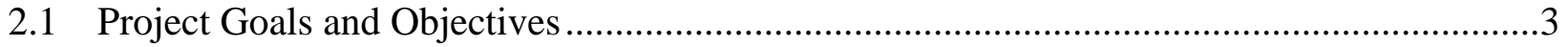

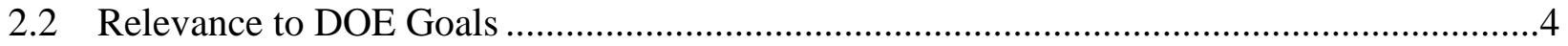

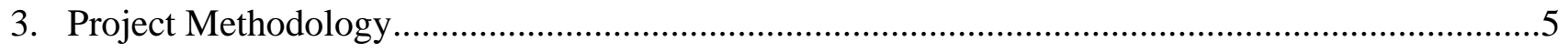

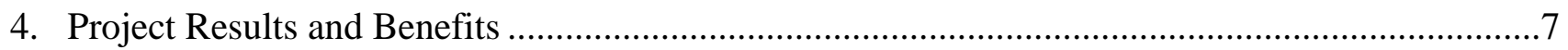

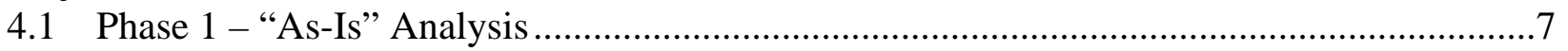

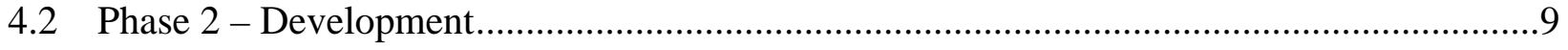

5. Using the Sustainable LED Product Design Guide …........................................................15

6. Conclusions and Opportunities for Further Work ..............................................................19

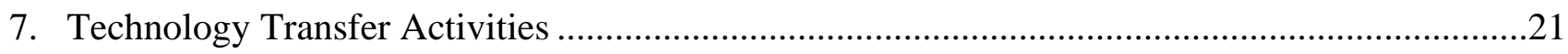

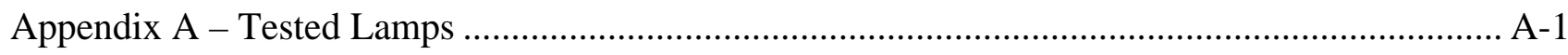

Appendix B - Environmental Impact Values and Documentation...............................................

Appendix C - Sustainable LED Product Design Guide ...........................................................

Appendix D - Presentation Materials ..................................................................................... D-1 



\section{Acronyms and Abbreviations}

\begin{tabular}{|c|c|c|c|}
\hline BOM & Bill-of-Materials & LCIA & life cycle impact assessment \\
\hline CFL & compact fluorescent lamp & LED & light-emitting diodes \\
\hline CRI & color rendering index & NCMS & $\begin{array}{l}\text { National Center for Manufacturing } \\
\text { Sciences }\end{array}$ \\
\hline DOE & Department of Energy & & \\
\hline EOL & end-of-life & REACH & $\begin{array}{l}\text { Registration, Evaluation, } \\
\text { Authorization and Restriction of } \\
\text { Chemical Substances }\end{array}$ \\
\hline EU & European Union & & \\
\hline GHG & greenhouse gas & RoHS & $\begin{array}{l}\text { Restriction of Hazardous } \\
\text { Substances }\end{array}$ \\
\hline LCA & life cycle assessment & SSL & solid state lighting \\
\hline LCI & life cycle impact & U.S. & United States \\
\hline
\end{tabular}





\section{Executive Summary}

Solid-state lighting, using light-emitting diodes (LEDs), has great energy saving potential over conventional incandescent and fluorescent lamps currently in use. However, although there are great energy savings in the use-phase of the lamps, the remainder of the product lifecycle is not so cut-and-dried. LED products rely on onboard or sometimes off-board power supplies and other electronic circuits. Aluminum heat sinks may be required for heat management to maintain long lifetimes. Adhesives or fasteners are necessary to hold all the constituent components together. All of this added complexity has an environmental footprint, especially when considered against the relative simplicity of fluorescent tubes and screw-in incandescent bulbs. Only by evaluating the entire product lifecycle, from raw material extraction to manufacturing to use through end-of-life, can the overall ecological impacts of the lamps be determined.

Ilumisys and the National Center for Manufacturing Sciences (NCMS) partnered on a three-year project awarded by the United States (U.S.) Department of Energy (DOE), to quantify the impacts of LED lamps, incandescent lamps and fluorescent benchmark lamps over a product lifecycle - i.e. to develop a sustainable design and manufacturing strategy that addresses product manufacturing, use, recycling and disposal scenarios for LED-based lighting. Based on the knowledge gained from extensive product tear-down studies of fluorescent and screw-in lighting products, lifecycle assessment tools, and accelerated lifecycle testing protocols, an interactive Sustainable LED Design Guide has been developed to aid architectural and lighting designers and engineers in making design decisions that consider three important environmental impacts (greenhouse gas emissions, energy use and mercury emission) across all phases of the life of an LED lighting product. Critical information developed for the lifecycle analysis and product feature comparisons is the useful life of the lighting product as well as its performance. The Design Guide is available at www.ncms.org, and was developed based on operational and durability testing of a variety of lighting products including power consumption, light output, and useful life of a lamp in order to allow a more realistic comparison of lamp designs.

This report describes the main project tasks, results and innovative features of the lifecycle assessment (LCA)-based design tools, and the key considerations driving the sustainable design of LED lighting systems. The Design Guide incorporates the following three novel features for efficiently evaluating LED lighting features in value-chains:

- Bill-of-Materials (BOM) Builder Designers may import process data for each component and supply functional data for the product, including power, consumption, lumen output and expected useful life.

- Environmental Impact Review - Designs are comparable across lifecycle phases, subsystems, and environmental impact category, and can be normalized to a userdefined functional unit.

- Drill-down Review - These provide an indepth look at individual lamp designs with the ability to review across subsystem or lifecycle phase.

As part of the dissemination effort, and to solicit input for validation of the Guide, Ilumisys and NCMS staff presented the project at five industry events, with a sixth technical presentation planned in late October 2011. 


\subsection{Project Partners}

Ilumisys is a Troy, Michigan-based company focused on next generation solid-state lighting technology. The company was formed in 2007 as a spinoff venture and wholly-owned subsidiary of Altair Engineering, Inc. with initial products based on Altair's intellectual property for the direct replacement of fluorescent light tubes with LED lamps.

NCMS is a not-for-profit organization, based in Ann Arbor, Michigan and a premier provider of collaborative research, information, knowledge and expertise to the North American manufacturing and defense community. Backed by over 300 corporate members, NCMS has spearheaded numerous advancements - in advanced materials, alternative energy, electronics, highperformance computing, rapid prototyping, nanomanufacturing, enterprise integration and sustainability - all focused on enhancing the nation's manufacturing competitiveness in the global economy. 


\section{Background}

It is estimated that lighting accounts for $25 \%$ of the total electricity usage by residential and commercial consumers in the U.S., and is responsible for $10 \%$ of all U.S. carbon dioxide emissions. The DOE estimates that lightingbased energy consumption can be reduced by at least $20 \%$ by 2030 through the use of solid-state LED based lighting. ${ }^{1}$ LEDs are the fastest growing category of lighting technology that can counter problems related to mercury and light quality which are inherent in fluorescent lighting systems. LEDs contain no mercury or hazardous materials, and consume up to $90 \%$ less energy with no significant radiant heat or ultraviolet radiation. There have been tremendous strides in LED technology and cost reduction in recent years, with many new lighting products entering the marketplace that claim to provide lumen outputs and color temperatures matching the brightness and quality of conventional light sources. There is a high level of concern in the U.S. LED Industry to take concerted steps to defend against poorquality LED products offering unwarranted claims of sustainability, in order to address allegations of "greenwashing." This project was organized to raise the industry stakeholders' interest in sustainability and environmental awareness, which are a necessary component of doing business, to assure the long-term competitiveness of the industry in the U.S. The project partners have addressed these issues by providing useful systems-level approaches and data-driven tools for guiding design and manufacturing decisions.

Preliminary investigations identified some key examples of design decisions that may influence the lifecycle impacts of the product that are

${ }^{1}$ Energy Savings Potential of Solid-State Lighting in General Illumination Applications 2010-2030. Prepared by Navigant Consulting, Inc. for the Department of Energy, Washington, DC, February 2010. comparable to the use-phase impacts. Among them were:

- Raw materials and manufacturing - LED lifetime is directly affected by ambient temperatures. As a result, LED lighting products require substantial heat-sinks of aluminum or other thermally conductive material. The cradle-to-gate energy required to produce the heat sink, which is estimated at approximately $200 \mathrm{MJ}$ per kg, is comparable to the energy consumed during several thousand hours of product use, and is thus, a significant factor in a lifecycle evaluation as proposed in this project.

- Upgradability - The ability to change out the LED devices for newer models while retaining much of the remaining fixture will have significant lifecycle advantages. However, this serviceability must be designed into the initial product, and involves tradeoffs in cost and convenience addressed in this project.

- End-of-life - LED lighting products do not contain mercury, and generally meet Restriction of Hazardous Substances (RoHS) compliance. However, LEDs do contain a wide variety of less common materials. New regulatory requirements, such as the REACH regulation in the European Union (EU), may affect the supply and availability of certain materials, and have consequences related to extended producer responsibility that will incur obligations for the manufacturer, and will limit disposal options for the consumer, at end-of-life.

This project analyzed the effect of such factors on the lifecycle performance of LED products. 


\subsection{Project Goals and Objectives}

While the solid-state lighting (SSL) LED technology itself provides the environmental benefits of reduced energy use and, by extension, greenhouse gas emissions in the usephase, the electronics, thermal management, and optical components have the potential to impact the environment during the other phases of the product lifecycle.

The main objective of this project was to evaluate alternative designs for solid-state lighting products over the entire product lifecycle with regard to key sustainability impact categories, including energy usage and greenhouse gas emissions. One expected outcome was the consolidation of key findings, measurements and lifecycle analyses for the development of a best practices guideline for eco-friendly LED product design that takes into account the current and future needs of LED lamps. By providing guidance to lighting designers, good design decisions can be made that help in reducing the environmental impact of the product in key phases: raw material extraction, product manufacturing, and end-oflife.

Rather than deliver a white paper outlining how to make good decisions or provide complicated lifecycle-based calculations, the partners opted to develop a public-domain Sustainable LED Product Design Guide via an online accessible web application that would simplify decisionmaking and be accessible to a broader audience of stakeholders. Allowing a user to develop simple BOM of their designs and get immediate visual feedback on the impacts of their material and process selection provides opportunities for quickly creating "what-if” scenarios. With the interactive ability to compare the sources of impacts by category, lifecycle phase, or product subsystems, a user could identify the primary sources of the impacts and design them out of the product.

\subsection{Relevance to DOE Goals}

The DOE Solid-State Lighting Multi-Year Program Plan (March 2006) specifically identifies cost (measured in dollars per kilolumen) and performance (measured in lumens per watt) as the immediate barriers to near-term adoption of solid-state lighting for general illumination applications. The global community is demanding that products be designed and manufactured in ways that help ensure sustainability by minimizing environmental impact throughout a product's lifecycle. Simultaneously, the lighting industry faces the daunting task of re-educating consumers and other manufacturing stakeholders on how to achieve greater sustainability and to produce the same amount of light with fewer watts.

The Sustainable LED Product Design Guide provides a widely-accessible, user-friendly tool which allows designers of LED lighting products to choose materials and processes based on reduced energy use, greenhouse gas emission, and mercury emission. The environmental impacts can be compared across multiple design concepts or against benchmark lighting products, and across lifecycle phases. These unique capabilities of the tool address the mission of the DOE SSL Program by helping designers to reduce lifecycle energy consumption of solid-state lighting products beyond just the use-phase, while also reducing exposure to environmental risk factors such as climate change and toxicity.

Beyond reduction of impacts through the selection of materials and manufacturing processes, performance characteristics of the lighting products are considered in the Guide, allowing the balance of design against light output, power draw, and lamp life e.g. the impacts associated with additional aluminum for thermal management might be far outweighed by a resulting extension of lamp life. 


\section{Project Methodology}

Life Cycle Assessment (LCA) is a methodology to develop an aggregate estimate of the environmental impacts by all of the processes that contribute to the raw materials, manufacturing, transportation, use, and end-of-life scenarios of a product or process.

The approach adopted to achieve the project objectives consisted of two project phases, executed in four technical tasks to perform systematic Life Cycle Impact Assessment (LCIA)-based database development and Design Guide development activities involving:

- Extensive product tear-downs of selected 16 LED, fluorescent and screw-in lighting products for material and manufacturing studies.

- Development of framework and databases for lifecycle assessment and impact information

- Accelerated lifecycle testing of products as input for design alternative exploration and analysis

- Development of user interface tools for dissemination of LCA-based sustainability information on the selected lighting products via web media.

The Statement of Work consisted of the following:
- Phase 1 - “As-Is” Analysis

Task 1.0 - Material and Manufacturing Analysis

Task 2.0 - Lifecycle Assessment

- Phase 2 - Development

Task 3.0 - Design Alternative Exploration and Analysis

Task 4.0 - Best Practices Guide Development

While the majority of technical tasks were performed at Ilumisys, overall project oversight and management was provided by NCMS.

Detailed quarterly progress reports were filed by NCMS during the project.

This final report provides the highlights of the above tasks with significant results that include:

(a) Test measurements and graphical results form product lifecycle testing (Appendix A)

(b) Environmental impact values derived (with key data sources) (Appendix B)

(c) User manual for the Sustainable LED Product Design Guide - a web-based tool hosted by NCMS at www.ncms.org (Appendix C)

(d) Sample slide presentation of the Guide for industry outreach (Appendix D). 



\section{Project Results and Benefits}

\subsection{Phase 1 - "As-Is" Analysis}

\section{Task 1.0 - Material and Manufacturing Analysis}

Ilumisys identified and procured 16 different product samples (screw-in incandescent bulbs and fluorescent lamps), and then performed systematic tear-down studies on each sample as part of the analysis work. The complete product list is provided in Appendix A.

BOM templates developed in Excel were populated with materials and manufacturing process information for these 16 different product samples so that LCA-based comparison studies could begin. The product list included three Ilumisys products for comparing to benchmarks during new product tests and BOM population.

The common lamp assembly structure developed that was used in completing the BOMs for all benchmark lamps consisted of the following five subsystems:

1. Electronic and Electrical Components

2. Optical Components

3. Thermal Management Components

4. Fixture Interface Components

5. Miscellaneous Mechanical Components.

All of the constituent processes in the raw material and manufacturing phases were identified to the best judgment of the Ilumisys engineers. For example, the heat sink for a given lamp might be identified as an aluminum casting with machining and anodizing operations. This would constitute aluminum extraction (raw material phase), aluminum casting (manufacturing phase), aluminum machining (manufacturing), and anodizing (manufacturing). The required measurable for these processes would be the part mass for the extraction, casting, and machining processes, and surface area for the anodizing process.

The focus of such analyses was on screw-in incandescent-replacement type lamps and 4-foot linear fluorescent replacement lamps. In each case, where possible, four units were purchased. Two of each model of the lamps were designated for testing, one was used for disassembly and one spare was kept for any other uses that might arise such as unexpected failures of test lamps. Initially, lamps that had been tested in the CALiPER program were selected so any additional data points might be available. As new products came on the market that might incorporate different design concepts or vastly improved function, these products might be purchased for analysis.

Once a lamp had been disassembled, its components were identified, classified into common subsystem designations, and measured for the lifecycle metrics appropriate for that component. This identified the use-phase impacts of the product.

Finally, an end-of-life scenario was assigned. The default assumption for all products was landfill. This was applied on the total product mass. LCA impacts were also available for incineration. With further development, scenarios could be generated for recycling and remanufacturing. These scenarios are more complex and would require further study.

An additional data point was required for each product in order to compare different products in a normalized manner. With the light output identified for each product, products can be compared based on a normalized functional unit, namely lumen-hours.

The power draw, light output, and life expectancy of the products were initially taken from manufacturer claims. It has been clear 
from the DOE sponsored CALiPER testing that manufacturer claims often differ from reality. Where possible, in the initial analyses, CALiPER results were used for light output and power draw. In order to determine consistent, long-term performance of the lamps, Ilumisys tested all products in their lighting lab. The details of the testing are outlined in the next section.

\section{Task 2.0 - Lifecycle Assessment}

Lifecycle assessment is a measure of the environmental impacts by all of the processes that contribute to the raw materials, manufacturing, transportation, use, and end-of-life scenarios of a product or process.

With NCMS support, information was extracted for populating the BOMs using query tools from two commercial life cycle impact (LCI) inventory databases: $\mathrm{GaBi}$ (PE database) and ecoInvent (SimaPro), and added to the Excel template - the majority of the lamp materials, other than electronics components, had data derived from $\mathrm{GaBi}$ that was included in the BOM template.

As the benchmark lamps were disassembled and cataloged, Ilumisys identified the materials and processes used to produce them, using $\mathrm{GaBi}$ and SimaPro LCI databases to provide "most representative" numbers as starting points for assessing the impacts of those materials and processes in three impact categories incorporated in the Material Data Feeder spreadsheet:

- Energy use (measured in $\mathrm{kW}$ )

- Greenhouse gas emissions (measured in 100-year equivalence of $\mathrm{kg} \mathrm{CO}_{2}$ )

- Mercury emissions (measured in mg).

Ilumisys and NCMS supplemented the information obtained from these two commercial databases by researching other independent measures of the inherent variability of the data, including such factors as:

- Variations in fuel heating value and carbon content

- Geographical variations in carbon intensity and electrical grid mix.

\section{Addressing Inherent Variability in LCI Data}

Periodic brainstorming meetings were held at NCMS with multi-disciplinary staff and internal sustainability and engineering experts at both organizations to identify possible strategies for calculating inherent and statistical variability and uncertainty in LCI data, so as to adopt robust methods for approximating the ranges of possible impact values. The goal was mainly to raise questions and thereby flag issues that may not have been fully considered. For example, when considering unaccounted carbon dioxide emissions from the core factors, SimaPro indicated $12.2 \mathrm{~kg} \mathrm{CO}_{2}$ emissions versus $\mathrm{PE}$ database's mean value was $8.9 \mathrm{~kg} \mathrm{CO}$.

Factors contributing to the variability in setting boundaries or ranges of the lifecycle impact categories (i.e. greenhouse gas emissions, energy and mercury emissions) included:

- Use of two different (independent) databases (ecoInvent and PE) often due to different methods, or missing values or results available from only one database)

- Variance of fuels (e.g. different coals have been very different heating values)

- Feedstocks (e.g. unknown locations for transportation of materials and products

- Transportation (unknown locations for transportation of materials and products)

- Process efficiency (well-defined versus vaguely defined processes, much data is based on European averages, whereas many of the lamps being evaluated are made in China). 
The NCMS-recommended strategy for setting ranges (obtained from the two commercial LCI databases) was to use a "minimum-of-theminimums, maximum-of-the-maximums" approach. This was done to approximate the inherent variability of impacts based on geography, process efficiency, etc. The primary driver to calculating this range was the variability of heating value and carbon content of the fossil fuels consumed in each of the processes. This approach, sometimes, did not account for the cases when data was only available from one of the two sources. In such cases, the "most representative" values for each impact were combined to develop a range, specified by a "maximum" (worst expected) and "minimum” (best feasible) value.

The BOM was set up in such a way that, as each product had its materials and processes identified, the lifecycle data was automatically filled in by way of a “data feeder” sheet. As LCI data was added and refined, the updates were thereby propagated immediately to all BOMs.

About $15 \%$ of cells in the Material Data Feeder Spreadsheet initially lacked data for the LCA model, for which the criticality of the "missing" data was assessed by Ilumisys. Examples of data needs that were resolved included:

- Tungsten (used in conventional bulb filaments)

- Silicone (used in adhesives and insulation from wiring used in LEDs) - Ilumisys required data on end-of-life stage for these materials.

\subsection{Phase 2 - Development}

\section{Task 3.0 - Design Alternative Exploration and Analysis}

Due to the volume of products included in durability tests, Ilumisys invested its own resources to develop an in-house laboratory so as to more efficiently and economically conduct light testing. This lab was operational in mid-
March 2010 with all testing components and instrumentation/data acquisition systems in place. Thereafter, product testing began in order to obtain real numbers for the functional lamp units under test.

Because the desired performance data included life expectancy of the lamps, long-term testing was needed to replace the manufacturer-claimed information. The Ilumisys team researched the test methods used in LM-79, LM-80, and Energy Star programs. Ilumisys employed a 5-foot diameter integrating sphere for testing linear fluorescent replacement lamps and a smaller 18” diameter integrating sphere for screw-in lamps. Lamps were stored in temperature-controlled boxes with a design based on the descriptions in Energy Star test documents. The box ambient temperature was maintained at $45^{\circ} \mathrm{C}$ and lamps were cycled continuously at 3 hours on, 20 minutes off (90\% ON, 10\% OFF). Four thermocouples were used in each box to assure that temperatures were maintained. Where necessary, 60W and 100W lamps were used to aid in maintaining the temperature. All lamps were separated by baffles as outlined in the Energy Star documents.

All lamps were "staged" outside their box for 1 hour prior to measure light output. They were left $\mathrm{ON}$ in $20^{\circ} \mathrm{C}$ ambient temperature in the lab before being moved quickly into the integrating sphere. Lamps were tested more frequently in the early part of the project (approximately twice per week) and less frequently later in the project (approximately twice per month). At the time of the writing of this report, lamps had been powered ON for approximately 8,000 hours (one calendar year of continuous use would be 8,760 hours).

The following metrics were collected in the product testing:

- Power consumption

- Current draw

- Power factor 
- Luminous flux (light output)

- Color rendering index (CRI)

- Color temperature

- Chromaticity.

To conduct photometric testing of the lamps, Ilumisys constructed a 62” diameter integrating sphere in which to place the lamps while the spectrometer took the light readings. The purpose of an integrating sphere was to disperse the light from the lamps so that there was an even distribution over the sphere surface so that the spectrometer could measure a nondirectional radiation of the light. Utilizing a standard lamp that was calibrated at an outside lab, Ilumisys was able to calibrate the integrating sphere setup.

Using the L70 specification of 70\% light output as the end-of-life condition, Ilumisys identified the real useful life of the lamps, either through direct measurement (if that condition was reached), or by extrapolation (if this condition was not reached within project timing). To this point we have used the manufacturer claims of light output and lifetime to define the functional units of the lifecycle analysis. This testing yielded more accurate numbers.
Samples of the charts compiled for a single lamp are shown in Figures 1 and 2. Charts of all tested lamps are provided in Appendix A.

As can be seen in Figure 1, curves were included showing manufacturer's claim (where applicable) and the Energy Star requirement for lamp life. For fluorescent lamps, the Energy Star life requirement was assumed to be 35,000 hours which is the commercial lamp requirement (note that at time of writing, an Energy Star standard has not been set for linear fluorescent replacement applications). For screw-in lamps, the 25,000 hour residential requirement was used. Also identified was the "L70" line which represents $70 \%$ of the initial lumen output. This is the generally accepted failure criteria for lighting. In cases where lamps did not fail within the timeframe of the project, data was extrapolated for populating the BOM with the other lamp information.

Chromaticity $X$ and $Y$ values identify the color temperature of the tested lamp. Figure 2 shows a scatter of the chromaticity values measured over the test period.

Lumens vs. Time - LED Fluor. Repl.

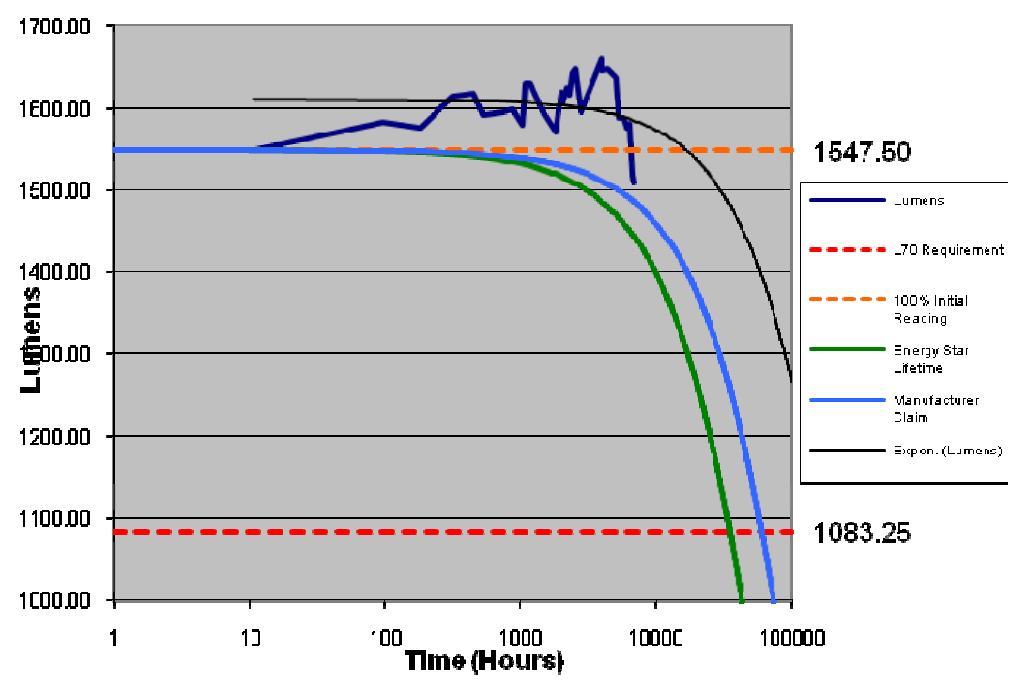

Figure 1. Example Lumens vs. Time Chart 


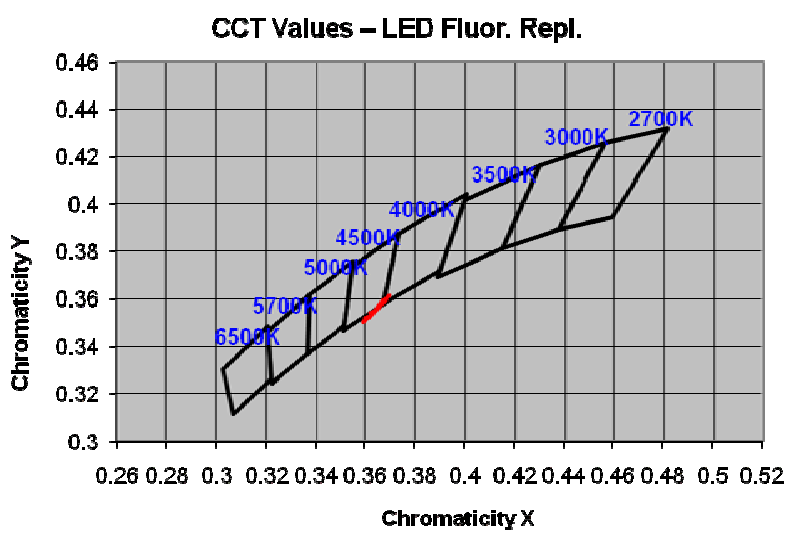

Figure 2. Example Chromaticity Chart

\section{LCA-Based Environmental Impact Database Generation}

A database of processes was generated by using the PE Database in $\mathrm{GaBi}$ and the ecoInvent Database in SimaPro. Processes expected to be used in the production of LED lamp components were identified in these databases and used to build a data feeder for the BOMs to be generated in the As-Is Analysis and in the Sustainable LED Product Design Guide tool. The data feeder sheet with the sources of the data cited is included in Appendix B.

The processes were divided into groupings corresponding to three lifecycle phases: production, use, and end-of-life. The production phase includes all raw material extraction and material processing processes. The use-phase represents the energy consumed in powering the lamp, and the end-of-life phase represents the disposal scenario for the lamp.

The environmental impacts chosen for measurement in this project were greenhouse gas emissions, energy consumption, and mercury emission.

Greenhouse gas emissions were chosen as an impact category due to the focus today on climate change. The oft-heard question about the environmental metric for products is "what is the carbon footprint?" It was clear that any designer would be interested in knowing the aspects of their design which contribute to this impact category.

Energy consumption is a category that seems to have great influence over many other categories. If the amount of energy required over the lifecycle is reduced, generally the greenhouse gas and toxic emissions are also reduced. Energy consumption was included in this Guide as an impact which could be focused on by the designer as a primary indicator of environmental impacts.

Finally, mercury emission was considered due to the relevance in the lighting industry because of its use in fluorescent lamps. Often in marketing LED products, the lack of mercury is touted as a benefit over linear fluorescent or compact fluorescent lamps (CFL). Including mercury in the As-Is Analysis and in the Guide would demonstrate the veracity of this claim. Though LED lamps do not contain mercury, mercury can be emitted to the environment in the production or use-phases of the lifecycle through the use of energy produced in fossil fuel power plants.

In the development of the database of impacts, data was not available for all expected processes that relate to the design of an LED lamp.

Additionally, some of the data seemed to be unrealistic. In some cases, surrogate data was used by means of studies of the lamps considered in the As-Is phase of the project.

For example, the LCA data for populated circuit boards is based on those assemblies as used in computer and telecommunications products. The circuit boards used these devices tend to be densely populated and contain many goldcontaining components. Gold production results in a high release of mercury according to the LCA documentation. Rather than use the data for populated circuit boards, the project team created new values based on an average of the individual electronic components for a representative sampling of products and used 
these new values in the data feeder for the Guide.

All impact values and documentation are included in Appendix B of this report.

\section{BOM Development}

A sample of each of the purchased lamps was disassembled and each of the components were weighed and tallied into the BOM. All components were divided into one of the following subsystems:

- Electrical and Electrical Components

- Optical Components

- Thermal Management Components

- Fixture Interface Components

- Miscellaneous Mechanical Components.

By associating each part with one of the above subsystems, lamps could be compared against common subsystems allowing the designer to understand the parts of the lamp assembly driving the highest impacts.

For each component entered into the BOM, the associated processes in producing that part were identified. Additionally, the energy consumed during the lamp's life was recorded by multiplying the measured power consumption by the measured (or extrapolated) life. The lamp was also assigned a disposal scenario.

All of the above information allowed impacts to be calculated against each of the lifecycle phases. Different products or concepts could then be compared against each phase of the lifecycle.

Another factor against which different products could be compared was in performance. By considering the lamp life and the lamp light output, different designs could be compared against different functional units. For example, two lamps could be compared on a basis of energy consumption per lumen or, greenhouse gas emissions per lumen-hour. This allowed products with different performance characteristics to be compared in a relative manner.

A more practical example was in the comparison of an LED screw-in lamp to that of a standard incandescent. An incandescent lightbulb has very low impacts in the production phase when compared to a much more complicated LED lamp. Additionally, the LED lamp may put out less light than the incandescent. However, the life of the LED lamp might be many times greater than that of the incandescent lamp.

Figure 3 illustrates the condition where an incandescent lamp puts out twice as much light as an LED lamp. This would suggest that since two LED lamps are required to achieve the same lighting levels, that the impacts then must be doubled with the LED lamp. However, in this example, we see that the life of the LED lamp is 30 times as long as that of the incandescent lamp. If the environmental impacts of these two lamps were compared per lumenhour, we would have to consider the impacts of 15 incandescent lamps to each LED lamp. Functional units are also valuable for a lamp designer to consider the benefits of adding material to make a lamp last longer or put out

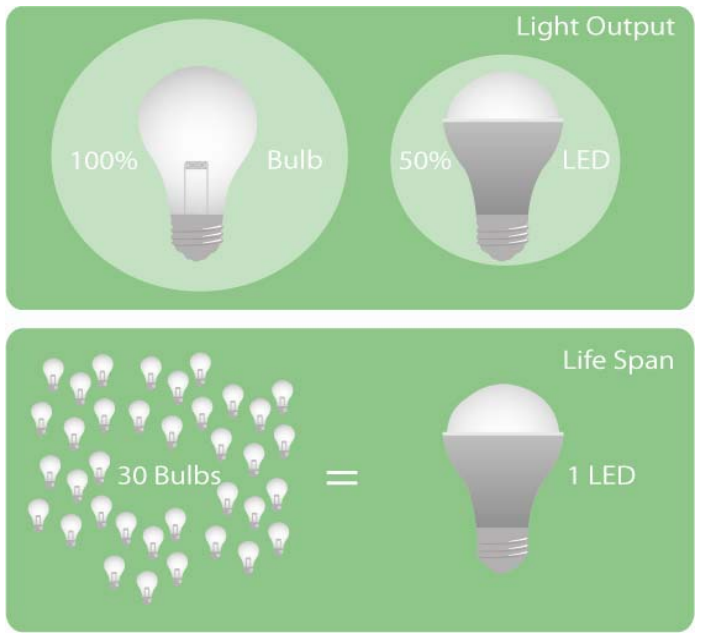

Figure 3. Illustration of the Application of Functional Units 
more light, and the resultant impacts on the environment.

\section{Trends Identified During As-Is Analysis}

Several general observations could be made about the environmental impacts associated with design attributes of the lamps. The greatest impacts came from the use-phase of the lamps from the energy consumed to power them. The use-phase accounted for the vast majority of the impacts.

In the production phase, it was seen that the electronics components in ballasted (self- or remotely-ballasted) lamps contributed greater impacts in general than other subsystems.
Other trends, such as the use of glass lenses rather than plastic, can be identified in review of the tested lamps.

Length of life of the lamps was another major determinant of impacts when lamps were compared against one another. For example, it might be worth adding additional heat sink mass or revising electronic components to lengthen life, even if it might mean greater productionphase impacts to those subsystems, if this could result in longer lamp life.

Task 4.0 - Best Practices Guide Development

Section 5 explains the Guide developed during the project in more detail. 



\section{Using the Sustainable LED Product Design Guide}

There are two sections to the Guide: BOM input and Reporting/Review. The Guide already contains data on existing lamp designs, including incandescent and fluorescent benchmarks, as well as LED lamps that were available during the course of the project.
First, we will review the BOM Building Sheet. Figure 4 shows the BOM sheet for a CFL bulb. In order to create a new BOM in the tool, the user first identifies some basic information about the lamp as shown in Figure 5.

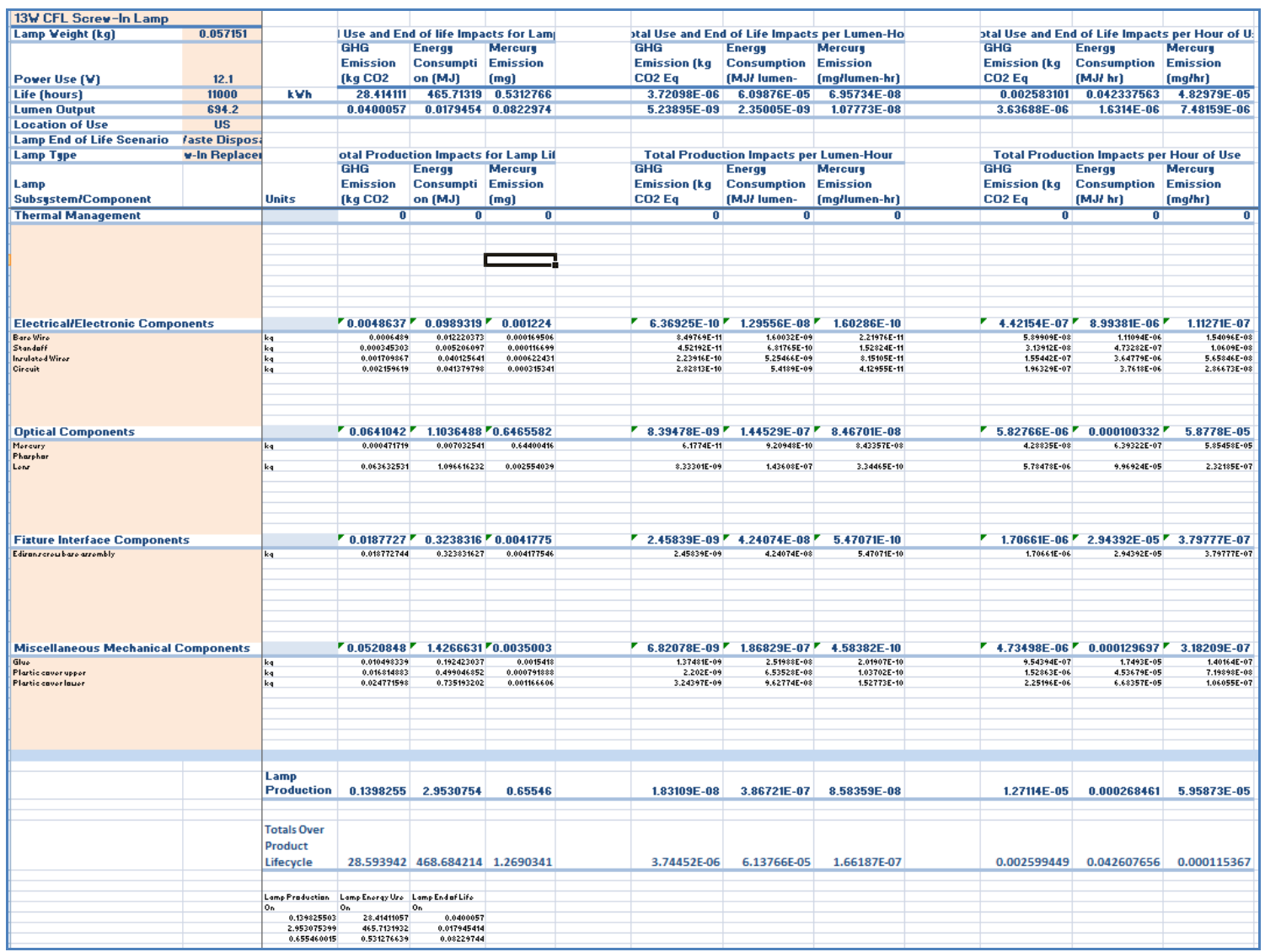

Figure 4. BOM Build Sheet for CFL Benchmark Bulb

\begin{tabular}{|lc|}
\hline 13W CFL Screw-In Lamp & \\
\hline Lamp Weight (kg) & 0.057151 \\
\hline & \\
\hline Power Use (W) & 12.1 \\
\hline Life (hours) & 11000 \\
\hline Lumen Output & 694.2 \\
\hline Location of Use & US \\
\hline Lamp End of Life Scenario & Waste Disposal \\
\hline Lamp Type & Screw-In Replacement \\
\hline
\end{tabular}

Figure 5. Lamp Information Panel 
The tan-highlighted cells indicate user input areas. The entries for power use, life, and lumen output for the pre-populated lamps in the Guide are based upon lab testing of the example lamps. The Life entry is based upon L70 failure criteria and in cases where tested lamps have not declined to $70 \%$ output, values are extrapolated on an exponential decay.

In the User-Defined lamp BOMs, the Lamp Type has been assigned already. In all example lamps, the End-of-Life Scenario is assumed to be landfill. Incineration is the other option. Any future extension of the project could allow recycling and remanufacturing scenarios to be included.

Next, the user may assign the components in their BOM to the subsystems defined in the sheet. These subsystems define the production phase of the product lifecycle.

In Figure 6, one example of a subsystem area on the BOM entry sheet is shown. The user identifies the components in that subsystem in the first column. In the following four columns, drop-down menus allow the material class, material, primary, and secondary processes to be assigned. The processes are discrete, so if a heat sink is made of cast aluminum and anodized, two line items must be created:

$$
\begin{aligned}
& \text { NonFerro -> Aluminum -> Cast -> None } \\
& \text { NonFerro -> Aluminum -> Cast -> Anodized }
\end{aligned}
$$

Next the user assigns the quantity of the line item within the assembly, and assigns a mass per component. This information allows the Guide to assign impacts to the production phase of the lamp design.

When the new BOM has been populated, the user may open the "LED Sustainable Design Guide" tab to review the impacts charts. The charts show the production, use, and end-of-life phases in different colors, with the production phase shown in different shades identifying the subsystems. A chart Control Panel allows the user to consider the total lamp lifecycle impacts or to compare lamps normalized to impacts per hour or impacts per lumen-hour. Using these controls, the user may identify the importance of light output and longevity of the lamp design in reducing the impacts over the lifecycle.

Additionally, the user may turn on or turn off each phase of the lifecycle to focus the data to the area most critical to the designer (Figure 7).

An example of the Greenhouse Gas Emission chart for screw-in lamps are shown in Figures 8 -10 , illustrating the effects of the chart controls.

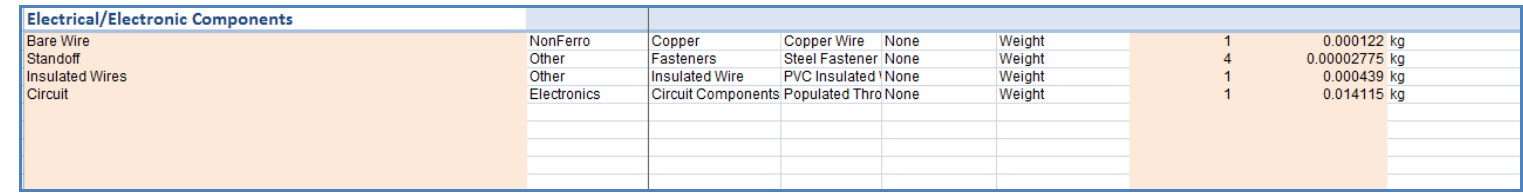

Figure 6. Example Subsystem Component Entry

\begin{tabular}{|l|l|}
\hline Chart Controls & Select from the drop-down menus below \\
\hline Total Impacts or Normalized & Total Impacts Over Lamp Life \\
\hline Include Lamp Production Phase? & Yes \\
\hline Include Lamp Use Phase? & Yes \\
\hline Include Lamp End of Life Phase? & Yes \\
\hline
\end{tabular}

Figure 7. Chart Control Panel 


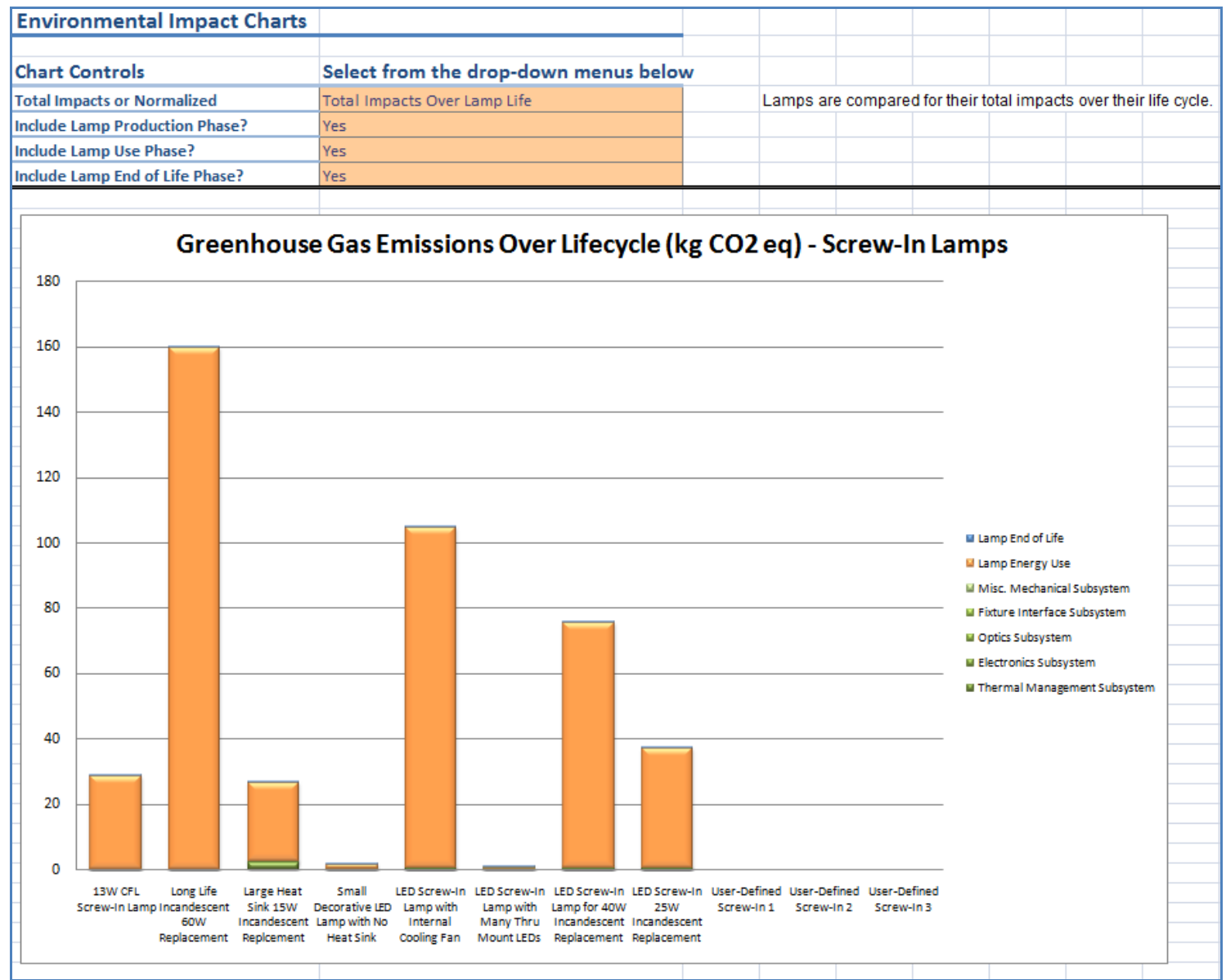

Figure 8. Greenhouse Gas Emissions Chart Showing Total Lifecycle Impacts

Figure 8 shows the total lifecycle impact for each of the lamps. Products with high power use and/or long life will show high impacts in the case in the use-phase. The use-phase is the dominant source of greenhouse gas emissions, and so little can be learned in this chart about the production and end-of-life phases.

Figure 9 shows the same chart as Figure 8, however, the use-phase data is omitted per the control panel. This allows a better look at the per-lamp impacts from the production and endof-life phases.

By looking at only the per-lamp impacts, we are not reviewing a complete picture. If we consider the longevity and/or the light output of the lamp, the user may make better design decisions. While there are very small impacts resulting from the production and disposal of an incandescent lightbulb due to its very basic design, the quantity of incandescent lightbulbs required to produce a given amount of light over the period of a very long-lasting LED lamp's life amplifies these impacts. By considering impacts normalized on a per lumen-hour basis, more information is available to the designer to make better design decisions.

Figure 10 illustrates the important of light output and longevity in reducing the per lumenhour impacts of a lamp.

Charts shown in the LED Sustainable Design Guide tab include Greenhouse Gas Emissions, Energy Use, and Mercury Emissions for ScrewIn and T8 lamps. All six charts will update simultaneously by manipulation of the control panel. 


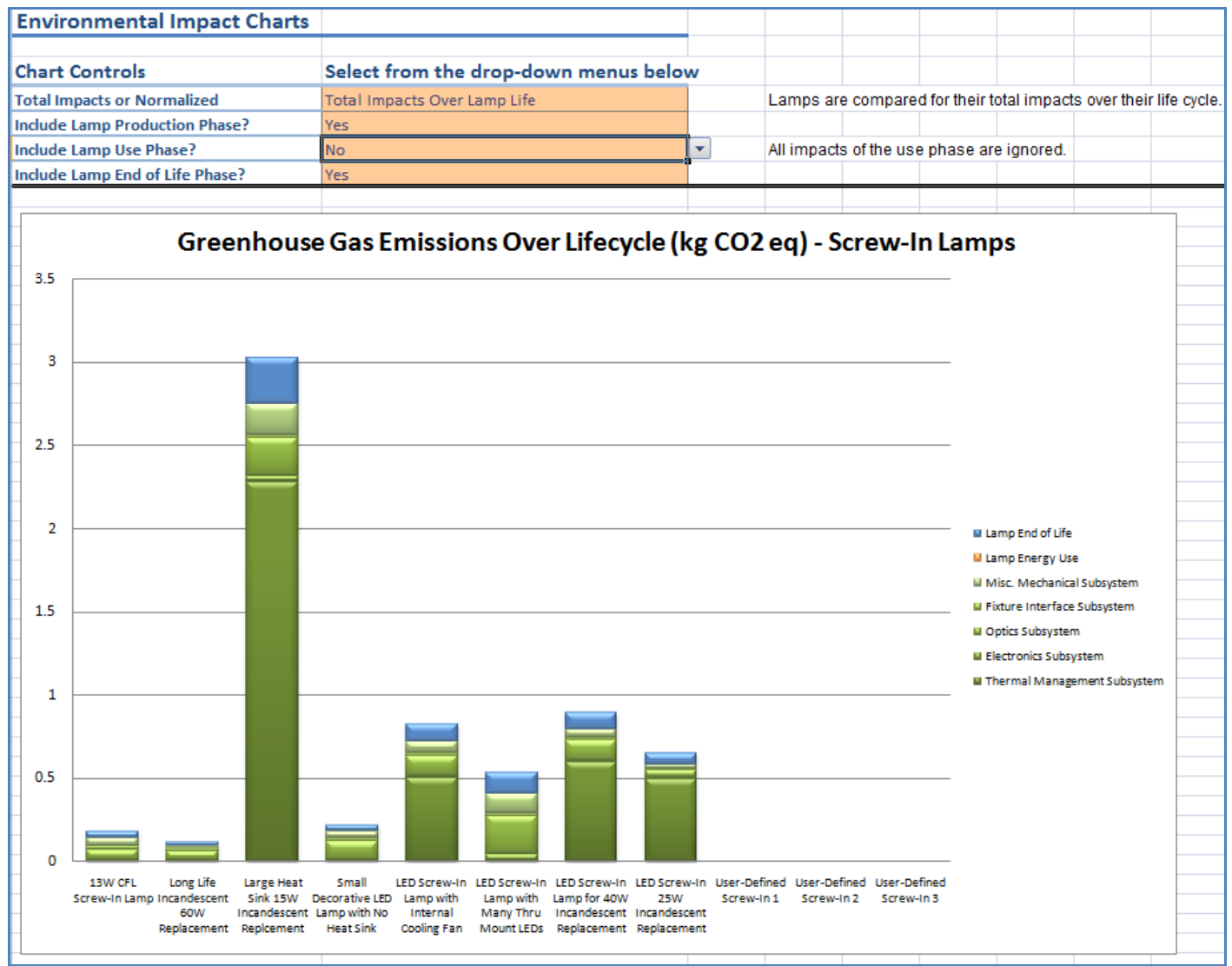

Figure 9. Greenhouse Gas Emissions Chart Showing Impacts in Production and End-of-Life Phases Only

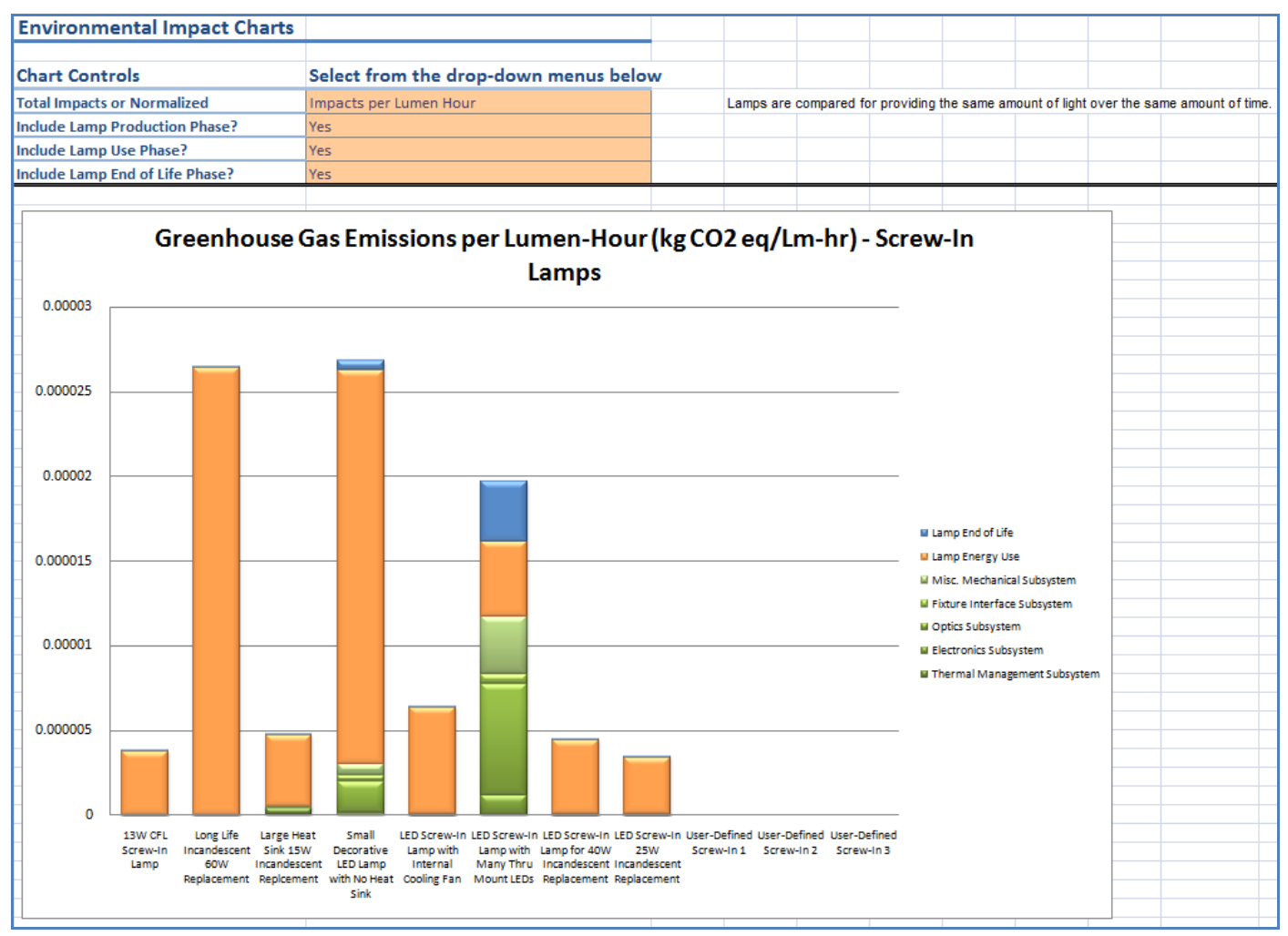

Figure 10. Greenhouse Gas Emissions Chart Showing All Lifecycle Phases Normalized to Lumen-Hours 


\section{Conclusions and Opportunities for Further Work}

\subsection{Process Database Improvements}

One of the limits of the Guide is the overall quality of the LCA data in the process database. In some cases, LCI data for some processes was either unavailable or inappropriate for the application. Often, these issues are present in the electrical circuit components. In particular, the LCI data for the LEDs that are most commonly used in quality LED lighting products is missing. Further LCA studies, beyond the default data available in SimaPro and $\mathrm{GaBi}$, would strengthen the underlying data feeding the Guide.

\subsection{Additional Impact Categories}

There are many other environmental impact categories that could be considered within the Guide with further research and data gathering. Water use, for example, is a category expected to be coming more to the forefront. This is a complex category which is the subject of much debate in how it is best evaluated. Other toxicity factors beyond mercury emission would add value to the Guide as well.

Further, a non-environmental, but still quite relevant impact of a product design is cost. Adding cost as a decision criteria alongside the environmental impacts would make the tool more useful to a designer in a real product design environment.

\subsection{Additional Lamp Form Factors}

Although the Guide, in its current form, should be useful for lamp form factors beyond screw-in replacement and linear fluorescent replacement lamps, further review of these other lamp types may identify other materials and processes that need to be added to the database. 



\section{Technology Transfer Activities}

\section{Publications/Presentations}

[1] "Sustainable LED Fluorescent Light Replacement Technology Project,” presentation by Dr. Manish Mehta at NIST Workshop on Sustainable Manufacturing, October 7, 2009, Gaithersburg, MD.

[2] Project information exchange regarding strategies for addressing inherent variability of LCI for LEDs and electronics held with Ms. Joel Todd, Chairperson of LEED Steering Committee, U.S. Green Building Council, January 28, 2010.

[3] “Achieving Sustainable LED Lighting: A Product Design Guide,” June 14 Presentation by James M. Amrine Jr., and Dr. Manish Mehta at NeoCon 2010, The Merchandize Mart, Chicago, IL.

[4] “Achieving Sustainable LED Lighting: A Product Design Guide,” invited presentation by James M. Amrine Jr., and Dr. Manish
Mehta to the South-East Michigan Sustainable Business Forum, September 16, 2010, Dearborn, MI.

[5] "A Product Design Guide for Achieving Sustainable LED Lighting Systems,” by James Amrine Jr. and Dr. Manish Mehta, presented at 2011 Electronics and Sustainability Symposium, March 23-24, 2011, Champaign, IL.

[6] "A Product Design Guide for Achieving Sustainable LED Lighting Systems,” by James Amrine Jr. and Dr. Manish Mehta, accepted for presentation at Michigan Green Chemistry Conference, October 27, 2011, Ann Arbor, MI. 



\section{Appendix A -Tested Lamps}

The following pages identify the lamps for which testing was conducted and against which the Guide was developed.

\begin{tabular}{|l|c|}
\hline Ilumisys Reference \# & FLUOR-001D \\
\hline Manufacturer & Philips \\
\hline Model & F40T12 Alto \\
\hline Description & 40W T12 Fluorescent Tube \\
\hline $\begin{array}{l}\text { Power Supply } \\
\text { Voltage/Device }\end{array}$ & $\begin{array}{c}\text { Philips Advance AmbiStar } \\
\text { RELB-1S40SC T12 Ballast } \\
\text { (120Vac Input) }\end{array}$ \\
\hline $\begin{array}{l}\text { Ilumisys Initial Test } \\
\text { Date }\end{array}$ & $8 / 17 / 10$ \\
\hline $\begin{array}{l}\text { Ilumisys Final/ } \\
\text { Recent Test Date: }\end{array}$ & $8 / 17 / / 11$ \\
\hline CALiPER Reference \# & $08-30$ \\
\hline CALiPER Test Round & 5 \\
\hline CALiPER Test Date & $5 / 08$ \\
\hline
\end{tabular}

\begin{tabular}{|l|c|c|c|}
\hline & $\begin{array}{c}\text { Manufacturer } \\
\text { Claim }\end{array}$ & $\begin{array}{c}\text { Test } \\
\text { Results }\end{array}$ & $\begin{array}{c}\text { CALiPER } \\
\text { Results }{ }^{\Delta}\end{array}$ \\
\hline $\begin{array}{l}\text { Initial Light Output } \\
\text { (Lumens) }\end{array}$ & 3,200 & 2,949 & 3,101 \\
\hline $\begin{array}{l}\text { 70\% Initial Output } \\
\text { (Lumens) }\end{array}$ & 2,240 & 2,064 & \\
\hline $\begin{array}{l}\text { Current Output } \\
\text { (Lumens) }\end{array}$ & 20,000 & 19,373 & \\
\hline $\begin{array}{l}\text { Life Expectancy } \\
\text { (Hours) }\end{array}$ & 4,100 & 4,768 & 2,884 \\
\hline $\begin{array}{l}\text { Correlated Color } \\
\text { Temperature (K) }\end{array}$ & 70 & 74 & 84 \\
\hline $\begin{array}{l}\text { Color Rendering } \\
\text { Index (Rg) }\end{array}$ & 40 & 40 & 39.0 \\
\hline Power (Watts) & & & \\
\hline
\end{tabular}

* Life expectancy for the test results is estimated using the exponential decay curve of the tested light output data and $70 \%$ of the initial output.

${ }_{\Delta}$ The color temperature of the CALiPER tested device was different than that of llumisys testing. 


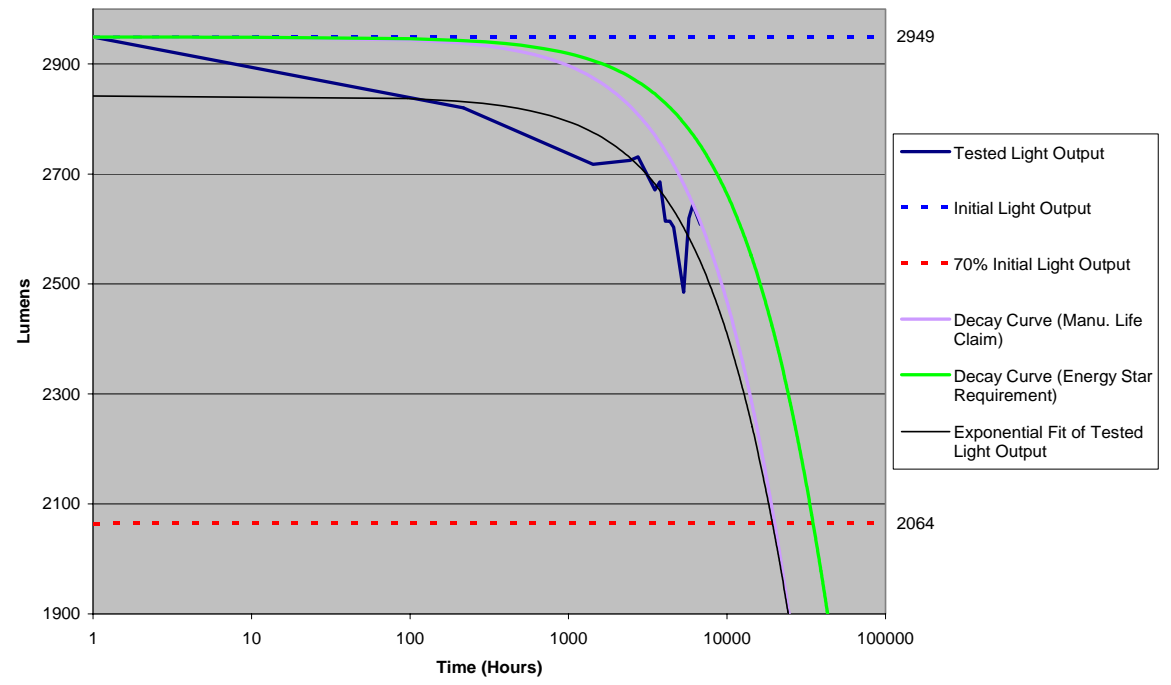

FLUOR-001D Lumens vs. Time

СCT Values

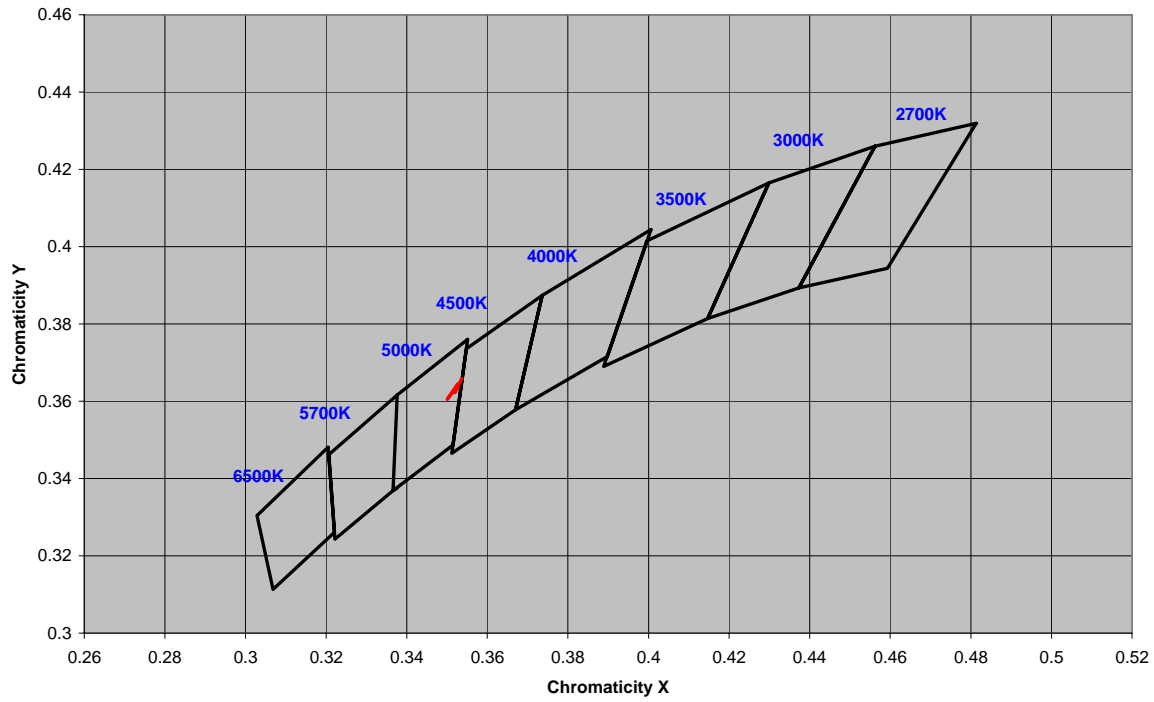

FLUOR-001D Chromacity Chart 


\begin{tabular}{|l|c|}
\hline Ilumisys Reference \# & FLUOR-002A \\
\hline Manufacturer & Mlumisys \\
\hline Model & MK1 \\
\hline Description & $\begin{array}{c}\text { Ballast Powered LED } \\
\text { Fluorescent-Replacement }\end{array}$ \\
\hline $\begin{array}{l}\text { Power Supply } \\
\text { Voltage/Device }\end{array}$ & $\begin{array}{r}\text { Centium ICN-1P32-N } \\
\text { Ilumisys Initial Test } \\
\text { Date }\end{array}$ \\
\hline $\begin{array}{l}\text { Ilumisys Final/ } \\
\text { Recent Test Date: }\end{array}$ & $4 / 14 / 10$ \\
\hline CALiPER Reference \# & $8 / 17 / 11$ \\
\hline CALiPER Test Round & $09-13 \mathrm{AB}$ \\
\hline CALiPER Test Date & 9 \\
\hline
\end{tabular}

\begin{tabular}{|l|c|c|c|}
\hline & $\begin{array}{c}\text { Manufacturer } \\
\text { Claim }\end{array}$ & $\begin{array}{c}\text { Test } \\
\text { Results }\end{array}$ & $\begin{array}{c}\text { CALiPER } \\
\text { Results }\end{array}$ \\
\hline $\begin{array}{l}\text { Initial Light Output } \\
\text { (Lumens) }\end{array}$ & 1,200 & 1,109 & 1,407 \\
\hline $\begin{array}{l}\text { 70\% Initial Output } \\
\text { (Lumens) }\end{array}$ & 840 & 776.3 & \\
\hline $\begin{array}{l}\text { Current Output } \\
\text { (Lumens) }\end{array}$ & 60,000 & 93,446 & \\
\hline $\begin{array}{l}\text { Life Expectancy } \\
\text { (Hours) }\end{array}$ & 4,200 & 4,294 & 3,758 \\
\hline $\begin{array}{l}\text { Correlated Color } \\
\text { Temperature (K) }\end{array}$ & 75 & 79 & 76 \\
\hline $\begin{array}{l}\text { Color Rendering } \\
\text { Index (R9) }\end{array}$ & 28 & 25 & 32 \\
\hline Power (Watts) & & & \\
\hline
\end{tabular}

* Life expectancy for the test results is estimated using the exponential decay curve of the tested light output data and $70 \%$ of the initial output.

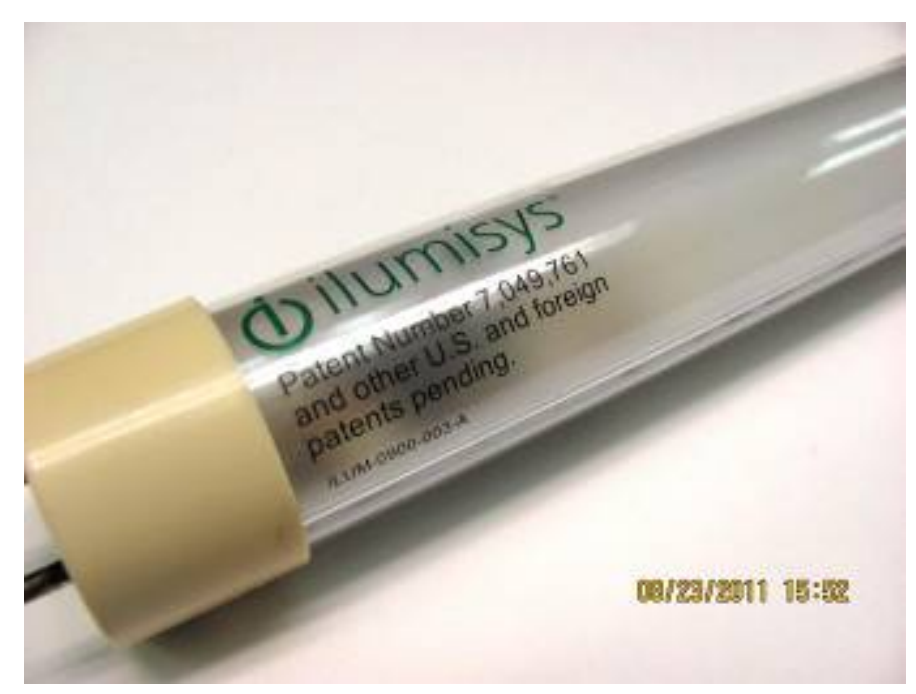




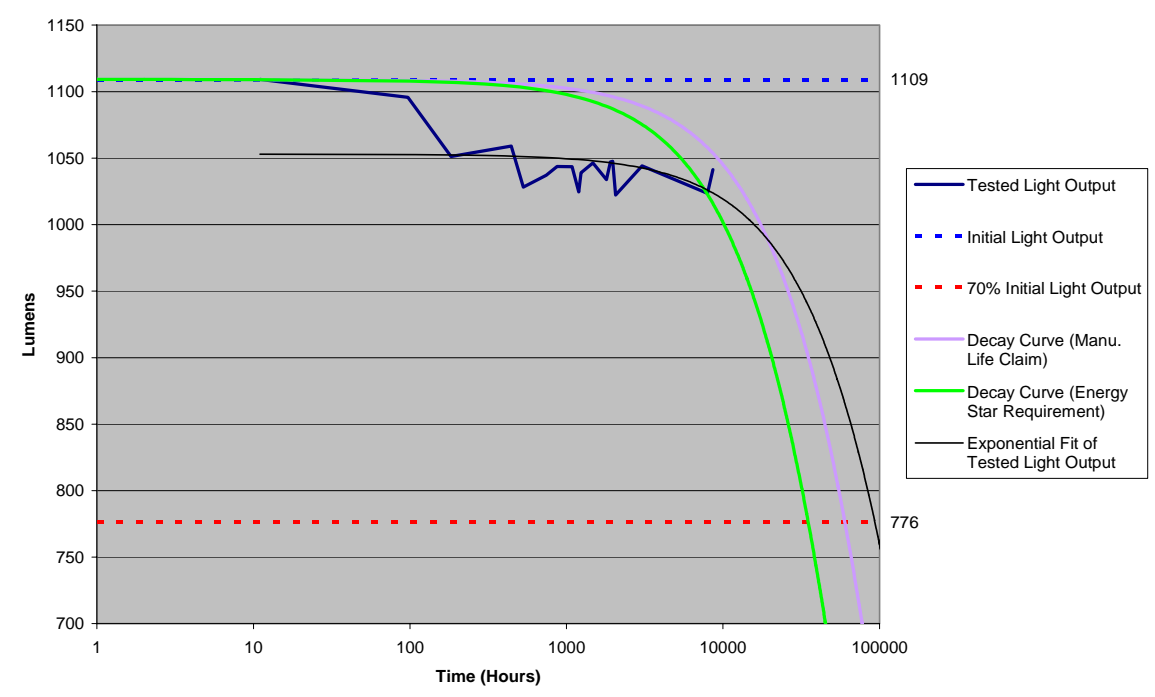

FLUOR-002A Lumens vs. Time

CCT Values

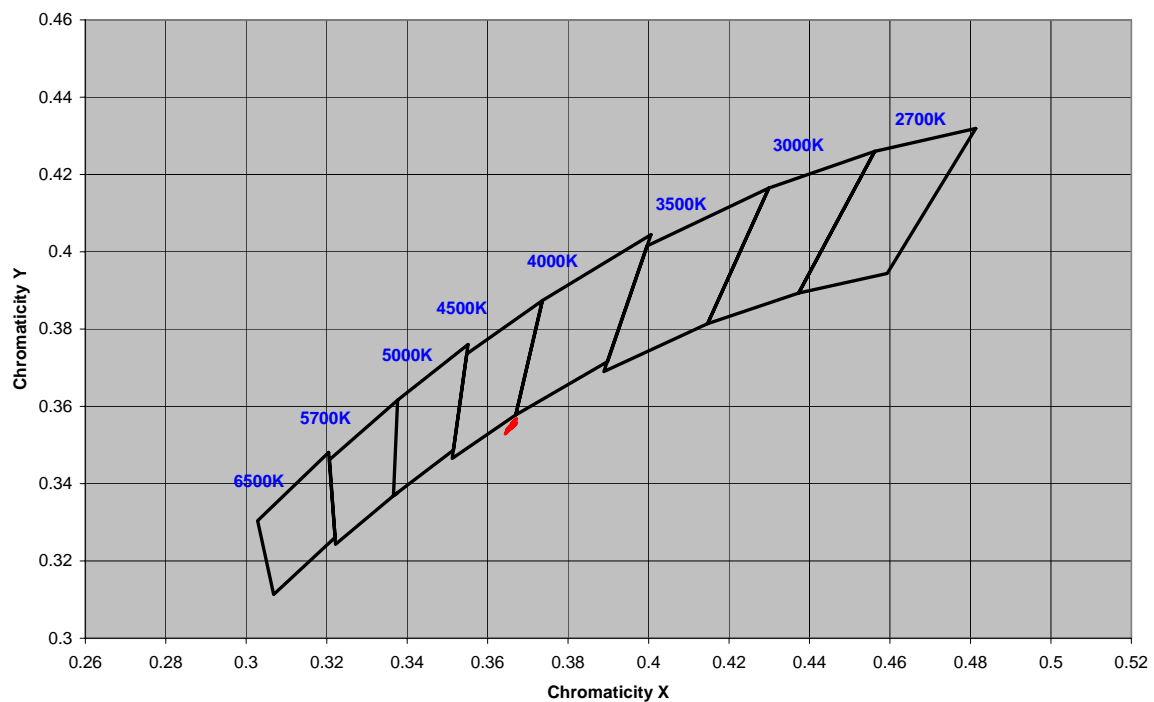

FLUOR-002A Chromacity Chart 


\begin{tabular}{|l|c|}
\hline Ilumisys Reference \# & FLUOR-003A \\
\hline Manufacturer & Ilumisys \\
\hline Model & MK2 \\
\hline Description & $\begin{array}{c}\text { Line Voltage LED Fluorescent- } \\
\text { Replacement }\end{array}$ \\
\hline $\begin{array}{l}\text { Power Supply } \\
\text { Voltage/Device }\end{array}$ & $4 / 14 / 10$ \\
\hline $\begin{array}{l}\text { llumisys Initial Test } \\
\text { Date }\end{array}$ & $8 / 17 / 11$ \\
\hline $\begin{array}{l}\text { Ilumisys Final/ } \\
\text { Recent Test Date: }\end{array}$ & $09-107$ \\
\hline CALiPER Reference \# & 11 \\
\hline CALiPER Test Round & $7 / 6 / 10$ \\
\hline CALiPER Test Date &
\end{tabular}

\begin{tabular}{|l|c|c|c|}
\hline & $\begin{array}{c}\text { Manufacturer } \\
\text { Claim }\end{array}$ & $\begin{array}{c}\text { Test } \\
\text { Results }\end{array}$ & $\begin{array}{c}\text { CALiPER } \\
\text { Results }\end{array}$ \\
\hline $\begin{array}{l}\text { Initial Light Output } \\
\text { (Lumens) }\end{array}$ & 1,500 & 1,575 & 1,539 \\
\hline $\begin{array}{l}\text { 70\% Initial Output } \\
\text { (Lumens) }\end{array}$ & 1,050 & 1,103 & \\
\hline $\begin{array}{l}\text { Current Output } \\
\text { (Lumens) }\end{array}$ & & 1,489 & \\
\hline $\begin{array}{l}\text { Life Expectancy* } \\
\text { (Hours) }\end{array}$ & 60,000 & 62,020 & \\
\hline $\begin{array}{l}\text { Correlated Color } \\
\text { Temperature (K) }\end{array}$ & 4,200 & 4,346 & 3,548 \\
\hline $\begin{array}{l}\text { Color Rendering } \\
\text { Index (R9) }\end{array}$ & 75 & 77 & 73 \\
\hline Power (Watts) & 22 & 22 & 22 \\
\hline
\end{tabular}

* Life expectancy for the test results is estimated using the exponential decay curve of the tested light output data and $70 \%$ of the initial output.

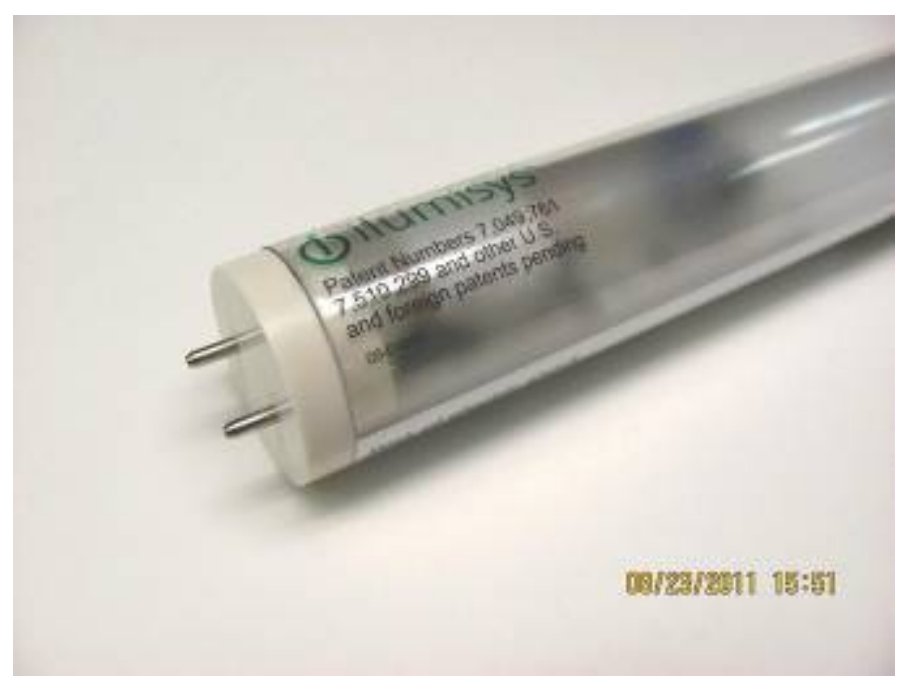




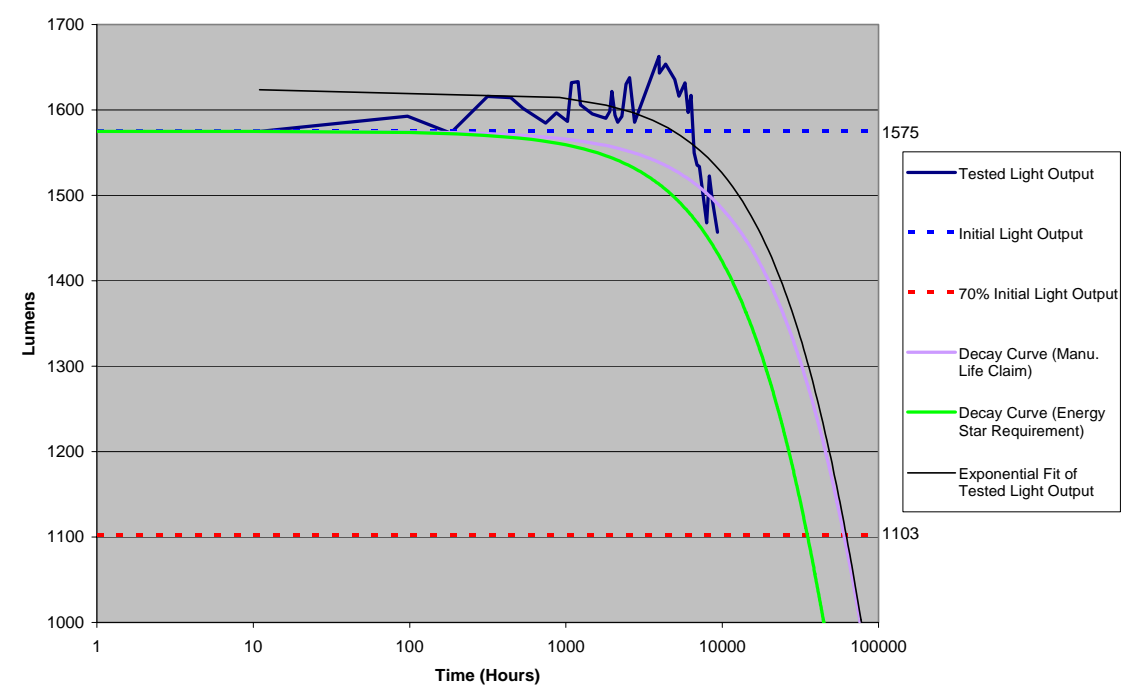

FLUOR-003A Lumens vs. Time

CCT Values

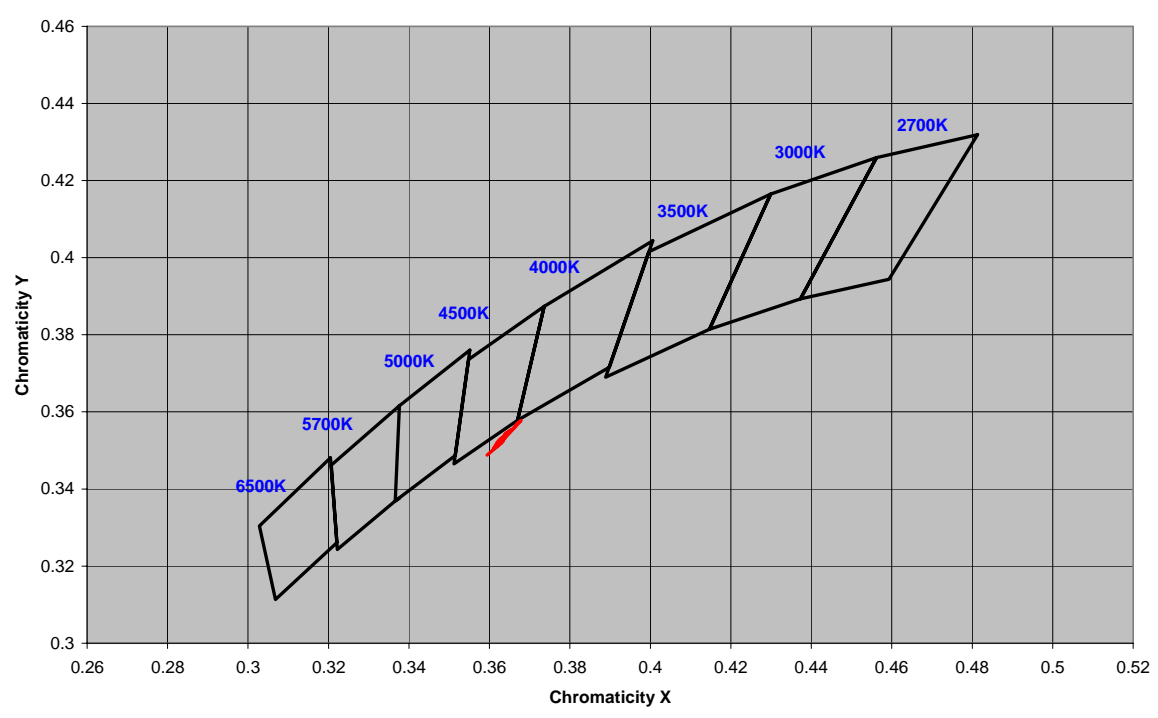

FLUOR-003A Chromacity Chart 


\begin{tabular}{|l|c|}
\hline Ilumisys Reference \# & FLUOR-004D \\
\hline Manufacturer & Philips \\
\hline Model & F32T8/TL841 Plus \\
\hline Description & $\begin{array}{c}\text { Philips Alto 32W T8 } \\
\text { Fluorescent Tube }\end{array}$ \\
\hline $\begin{array}{l}\text { Power Supply } \\
\text { Voltage/Device }\end{array}$ & $\begin{array}{c}\text { Philips Advance Centium ICN- } \\
\text { 1P32-N T8 Ballast 120-277 Vac }\end{array}$ \\
\hline $\begin{array}{l}\text { Ilumisys Initial Test } \\
\text { Date }\end{array}$ & $4 / 14 / 10$ \\
\hline $\begin{array}{l}\text { llumisys Final/ } \\
\text { Recent Test Date: }\end{array}$ & $8 / 17 / 11$ \\
\hline CALiPER Reference \# & $08-28$ \\
\hline CALiPER Test Round & 5 \\
\hline CALiPER Test Date & $5 / 08$ \\
\hline
\end{tabular}

\begin{tabular}{|l|c|c|c|}
\hline & $\begin{array}{c}\text { Manufacturer } \\
\text { Claim }\end{array}$ & $\begin{array}{c}\text { Test } \\
\text { Results }\end{array}$ & $\begin{array}{c}\text { CALiPER } \\
\text { Results }\end{array}$ \\
\hline $\begin{array}{l}\text { Initial Light Output } \\
\text { (Lumens) }\end{array}$ & 2,950 & 2,681 & 3,081 \\
\hline $\begin{array}{l}70 \% \text { Initial Output } \\
\text { (Lumens) }\end{array}$ & 2,065 & 1,877 & \\
\hline $\begin{array}{l}\text { Current Output } \\
\text { (Lumens) }\end{array}$ & 39,000 & 40,790 & \\
\hline $\begin{array}{l}\text { Life Expectancy* } \\
\text { (Hours) }\end{array}$ & 4,100 & 3,829 & 3,932 \\
\hline $\begin{array}{l}\text { Correlated Color } \\
\text { Temperature (K) }\end{array}$ & 85 & 78 & 81 \\
\hline $\begin{array}{l}\text { Color Rendering } \\
\text { Index (R9) }\end{array}$ & 32 & 32 & 32 \\
\hline Power (Watts) & & & \\
\hline
\end{tabular}

* Life expectancy for the test results is estimated using the exponential decay curve of the tested light output data and $70 \%$ of the initial output. 


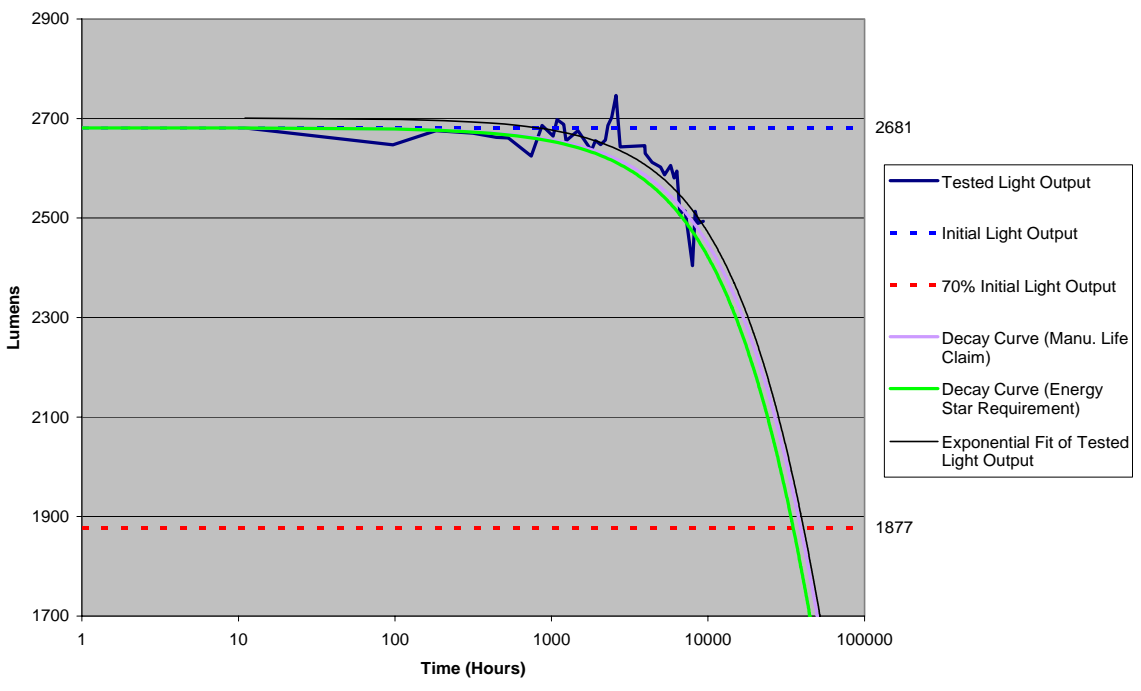

FLUOR-004D Lumens vs. Time

CCT Values

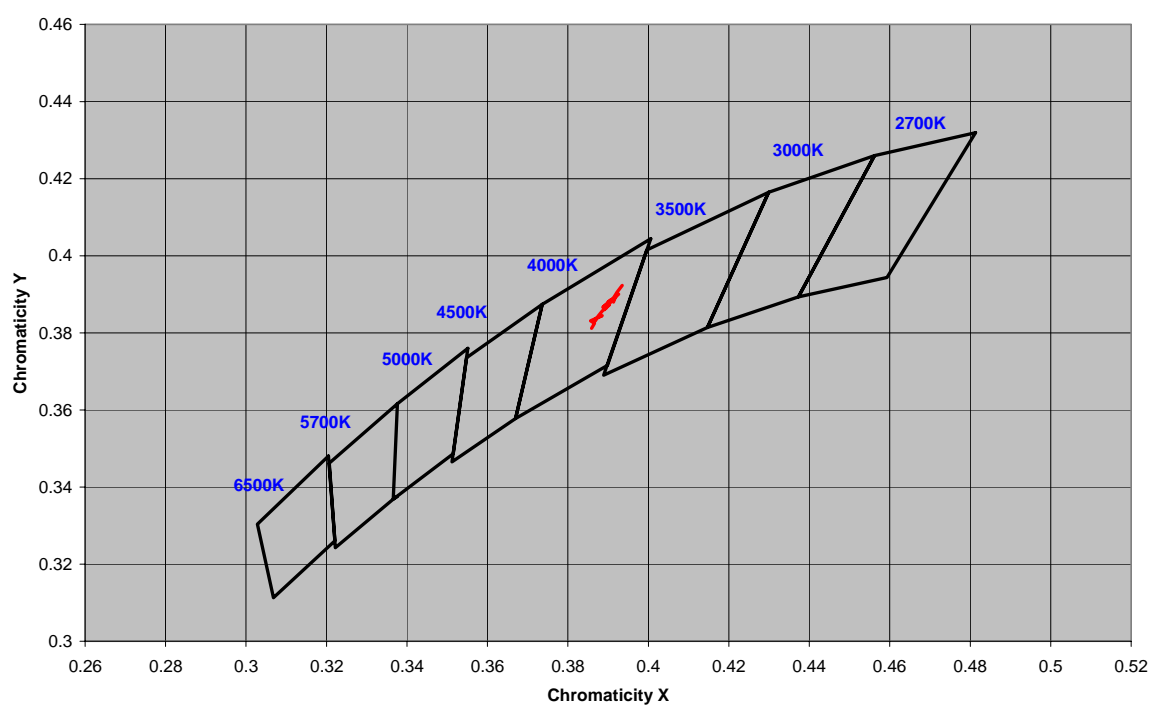

FLUOR-004D Chromacity Chart 


\begin{tabular}{|l|c|}
\hline Ilumisys Reference \# & FLUOR-005C \\
\hline Manufacturer & SeeSmart LED, Inc. \\
\hline Model & $\begin{array}{r}\text { 2700K LED Tube Light 15W } \\
\text { Clear Lens Warm White LED } \\
\text { Fescription }\end{array}$ \\
\hline $\begin{array}{l}\text { Power Supply } \\
\text { Voltage/Device }\end{array}$ & 120Vac \\
\hline $\begin{array}{l}\text { llumisys Initial Test } \\
\text { Date }\end{array}$ & $4 / 16 / 10$ \\
\hline $\begin{array}{l}\text { Ilumisys Final/ } \\
\text { Recent Test Date: }\end{array}$ & $8 / 17 / 11$ \\
\hline CALiPER Reference \# & $09-04$ \\
\hline CALiPER Test Round & 9 \\
\hline CALiPER Test Date & $6 / 09$ \\
\hline
\end{tabular}

\begin{tabular}{|l|c|c|c|}
\hline & $\begin{array}{c}\text { Manufacturer } \\
\text { Claim }\end{array}$ & $\begin{array}{c}\text { Test } \\
\text { Results }\end{array}$ & $\begin{array}{c}\text { CALiPER } \\
\text { Results }\end{array}$ \\
\hline $\begin{array}{l}\text { Initial Light Output } \\
\text { (Lumens) }\end{array}$ & 1,500 & 1,216 & 1,218 \\
\hline $\begin{array}{l}70 \% \text { Initial Output } \\
\text { (Lumens) }\end{array}$ & 1,050 & 851 & \\
\hline $\begin{array}{l}\text { Current Output } \\
\text { (Lumens) }\end{array}$ & 100,000 & 24,400 & \\
\hline $\begin{array}{l}\text { Life Expectancy* } \\
\text { (Hours) }\end{array}$ & 2,700 & 3,403 & 3,221 \\
\hline $\begin{array}{l}\text { Correlated Color } \\
\text { Temperature (K) }\end{array}$ & - & 70 & 66 \\
\hline $\begin{array}{l}\text { Color Rendering } \\
\text { Index (R9) }\end{array}$ & 15 & 16 & 16 \\
\hline Power (Watts) & & & \\
\hline
\end{tabular}

* Life expectancy for the test results is estimated using the exponential decay curve of the tested light output data and $70 \%$ of the initial output.

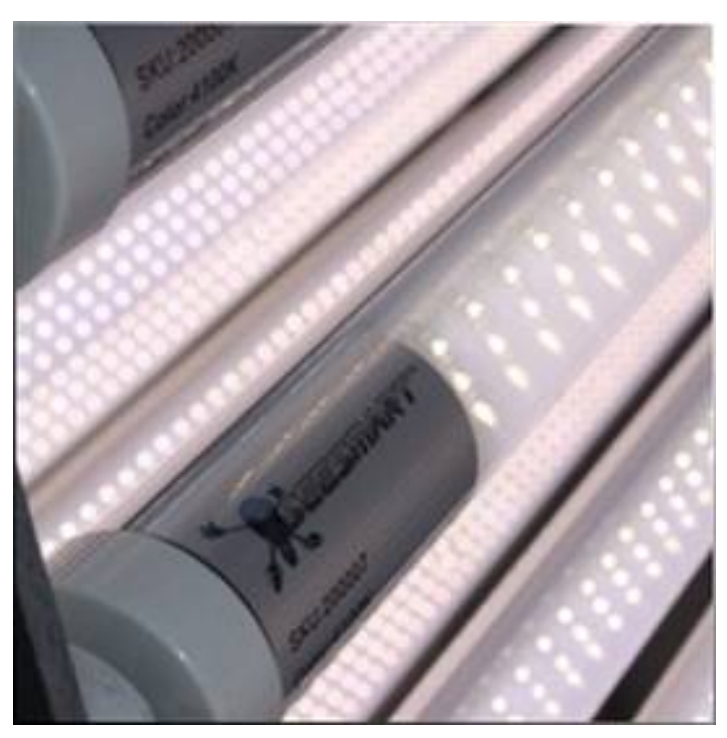




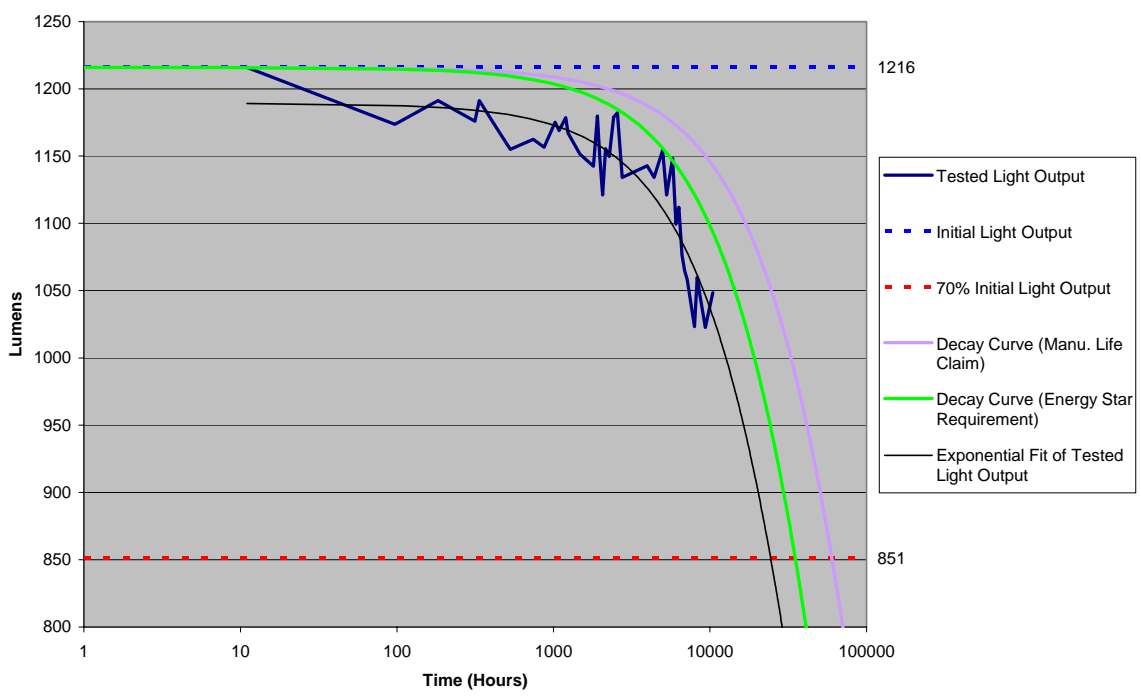

FLUOR-005C Lumens vs. Time

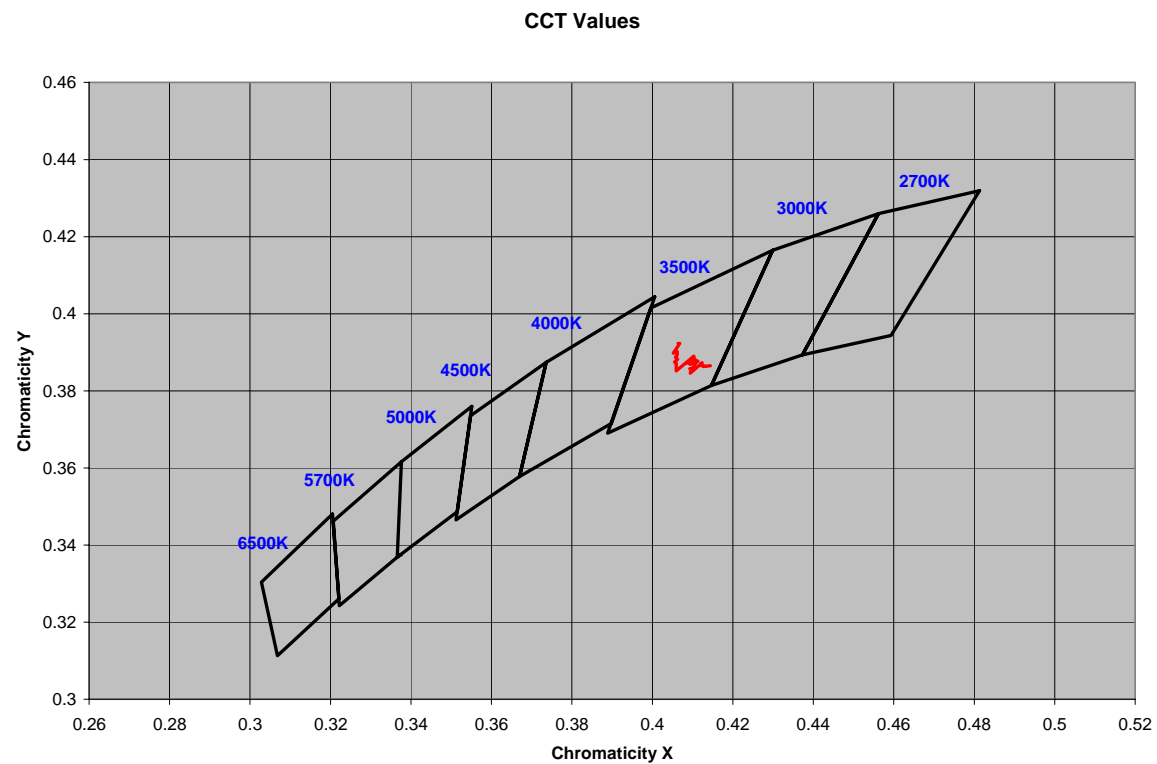

FLUOR-005C Chromacity Chart 


\begin{tabular}{|l|c|}
\hline Ilumisys Reference \# & FLUOR-007A \\
\hline Manufacturer & Mlumisys \\
\hline Model & $\begin{array}{c}\text { Line-Voltage LED Fluorescent- } \\
\text { Replacement (Glass) }\end{array}$ \\
\hline Description & 120Vac \\
\hline $\begin{array}{l}\text { Power Supply } \\
\text { Voltage/Device }\end{array}$ & $4 / 14 / 10$ \\
\hline $\begin{array}{l}\text { llumisys Initial Test } \\
\text { Date }\end{array}$ & $8 / 17 / 11$ \\
\hline $\begin{array}{l}\text { Ilumisys Final/ } \\
\text { Recent Test Date: }\end{array}$ & - \\
\hline CALiPER Reference \# & - \\
\hline CALiPER Test Round & - \\
\hline CALiPER Test Date & \\
\hline
\end{tabular}

\begin{tabular}{|l|c|c|c|}
\hline & $\begin{array}{c}\text { Manufacturer } \\
\text { Claim }\end{array}$ & $\begin{array}{c}\text { Test } \\
\text { Results }\end{array}$ & $\begin{array}{c}\text { CALiPER } \\
\text { Results }\end{array}$ \\
\hline $\begin{array}{l}\text { Initial Light Output } \\
\text { (Lumens) }\end{array}$ & 1,200 & 1,714 & - \\
\hline $\begin{array}{l}\text { 70\% Initial Output } \\
\text { (Lumens) }\end{array}$ & 840 & 1,200 & \\
\hline $\begin{array}{l}\text { Current Output } \\
\text { (Lumens) }\end{array}$ & & 1,611 & \\
\hline $\begin{array}{l}\text { Life Expectancy } \\
\text { (Hours) }\end{array}$ & 60,000 & 28,831 & \\
\hline $\begin{array}{l}\text { Correlated Color } \\
\text { Temperature (K) }\end{array}$ & 3,500 & 3,499 & - \\
\hline $\begin{array}{l}\text { Color Rendering } \\
\text { Index (R.) }\end{array}$ & 70 & 70 & - \\
\hline Power (Watts) & 22 & 25 & - \\
\hline
\end{tabular}

* Life expectancy for the test results is estimated using the exponential decay curve of the tested light output data and $70 \%$ of the initial output. 


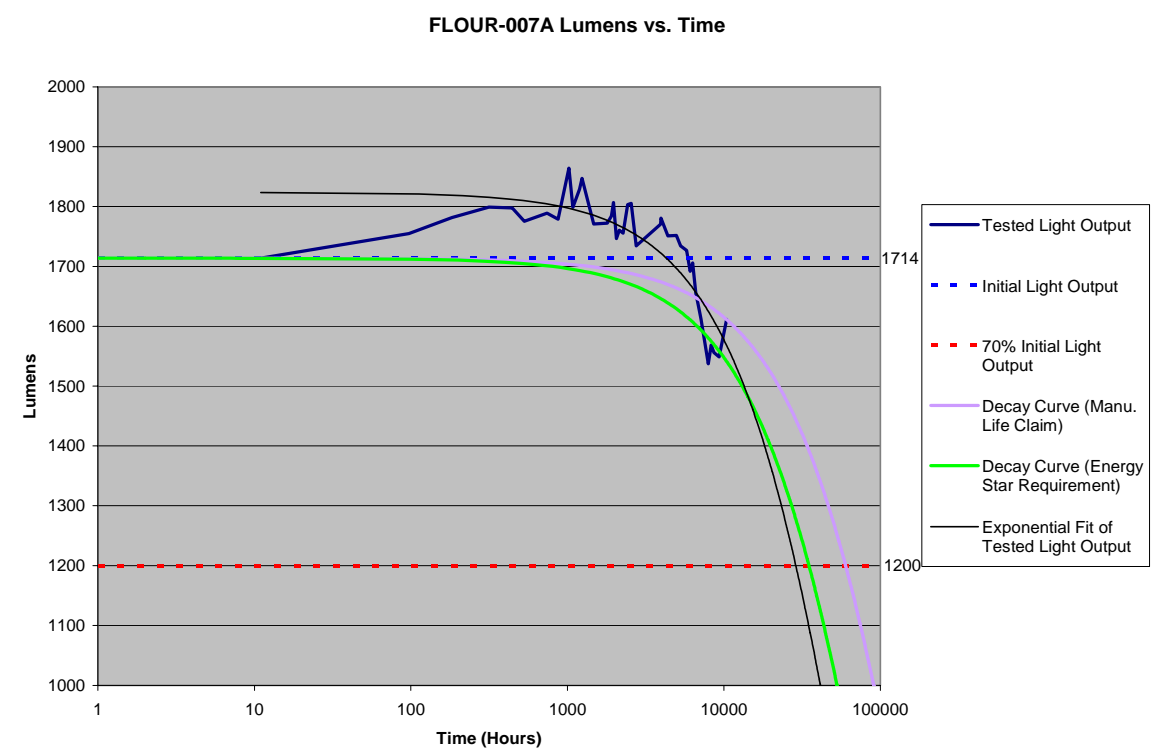

FLUOR-007A Lumens vs. Time

CCT Values

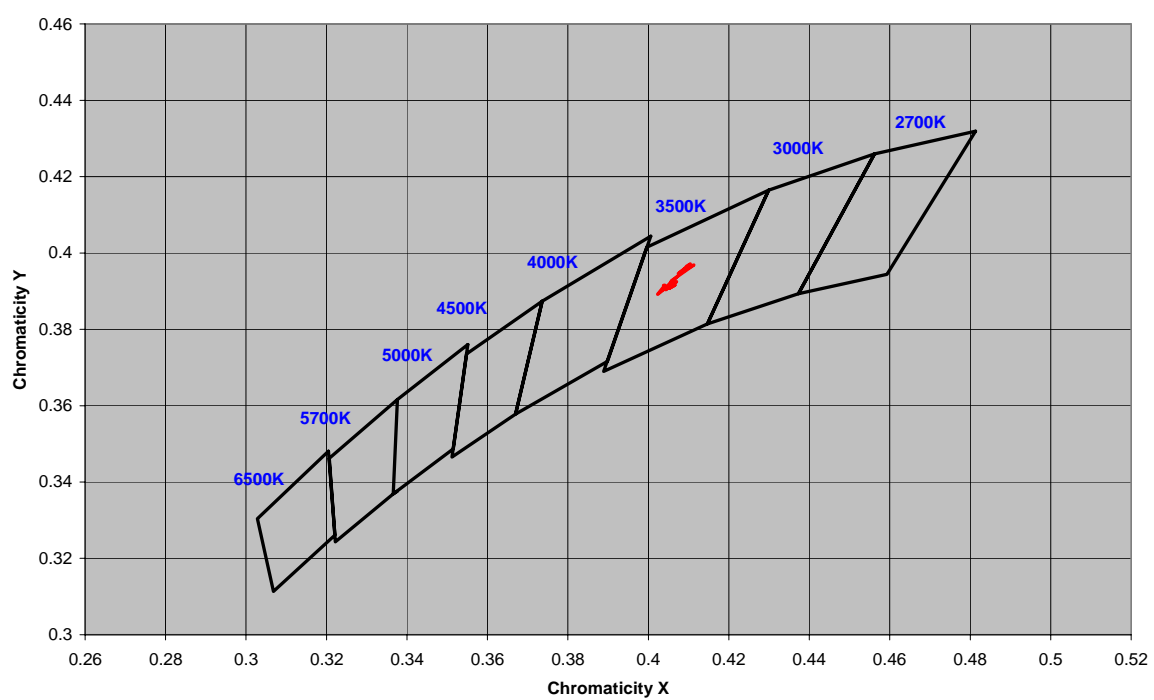

FLUOR-007A Chromacity Chart 


\begin{tabular}{|l|c|}
\hline Ilumisys Reference \# & FLUOR-010D \\
\hline Manufacturer & Lead Sun \\
\hline Model & $\begin{array}{c}\text { 15W External Driver LED } \\
\text { Fluorescent-Replacement }\end{array}$ \\
\hline Description & $\begin{array}{c}\text { LS472331.0 100-240Vac Input - } \\
\text { 15-34V/480mA Output } \\
\text { (Proprietary LED Driver) }\end{array}$ \\
\hline $\begin{array}{l}\text { Power Supply } \\
\text { Voltage/Device }\end{array}$ & $4 / 16 / 10$ \\
\hline $\begin{array}{l}\text { Ilumisys Initial Test } \\
\text { Date }\end{array}$ & $5 / 28 / 11$ \\
\hline $\begin{array}{l}\text { Ilumisys Final/ } \\
\text { Recent Test Date: }\end{array}$ & - \\
\hline CALiPER Reference \# & - \\
\hline CALiPER Test Round & - \\
\hline CALiPER Test Date & \\
\hline
\end{tabular}

\begin{tabular}{|l|c|c|c|}
\hline & $\begin{array}{c}\text { Manufacturer } \\
\text { Claim }\end{array}$ & $\begin{array}{c}\text { Test } \\
\text { Results }\end{array}$ & $\begin{array}{c}\text { CALiPER } \\
\text { Results }\end{array}$ \\
\hline $\begin{array}{l}\text { Initial Light Output } \\
\text { (Lumens) }\end{array}$ & 1,400 & 1,385 & \\
\hline $\begin{array}{l}\text { 70\% Initial Output } \\
\text { (Lumens) }\end{array}$ & 980 & 970 & \\
\hline $\begin{array}{l}\text { Current Output } \\
\text { (Lumens) }\end{array}$ & - & 1,504 & \\
\hline $\begin{array}{l}\text { Life Expectancy* } \\
\text { (Hours) }\end{array}$ & $-6,668^{a}$ & \\
\hline $\begin{array}{l}\text { Correlated Color } \\
\text { Temperature (K) }\end{array}$ & $4,500-5,500$ & & \\
\hline $\begin{array}{l}\text { Color Rendering } \\
\text { Index (R9) }\end{array}$ & - & 18 & \\
\hline Power (Watts) & 15 & & \\
\hline
\end{tabular}

* Life expectancy for the test results is estimated using the exponential decay curve of the tested light output data and $70 \%$ of the initial output.

a This bulb was incidentally damaged at this level many hours before it reached its end of life and testing was complete.

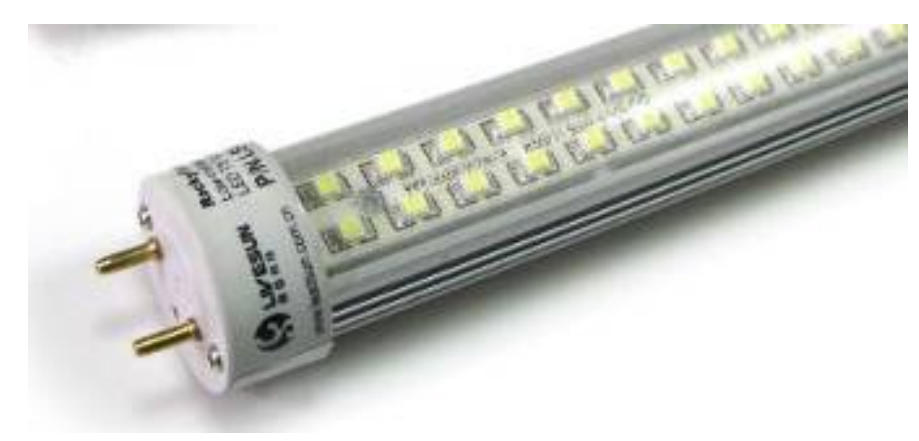




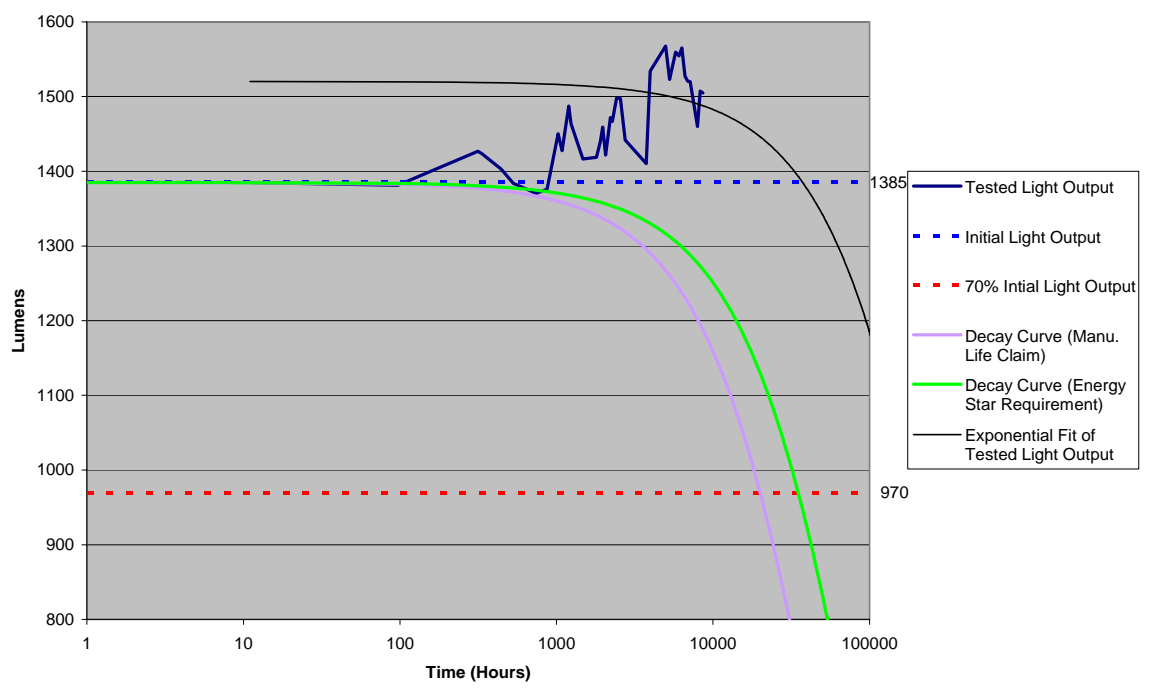

FLUOR-010D Lumens vs. Time

CCT Values

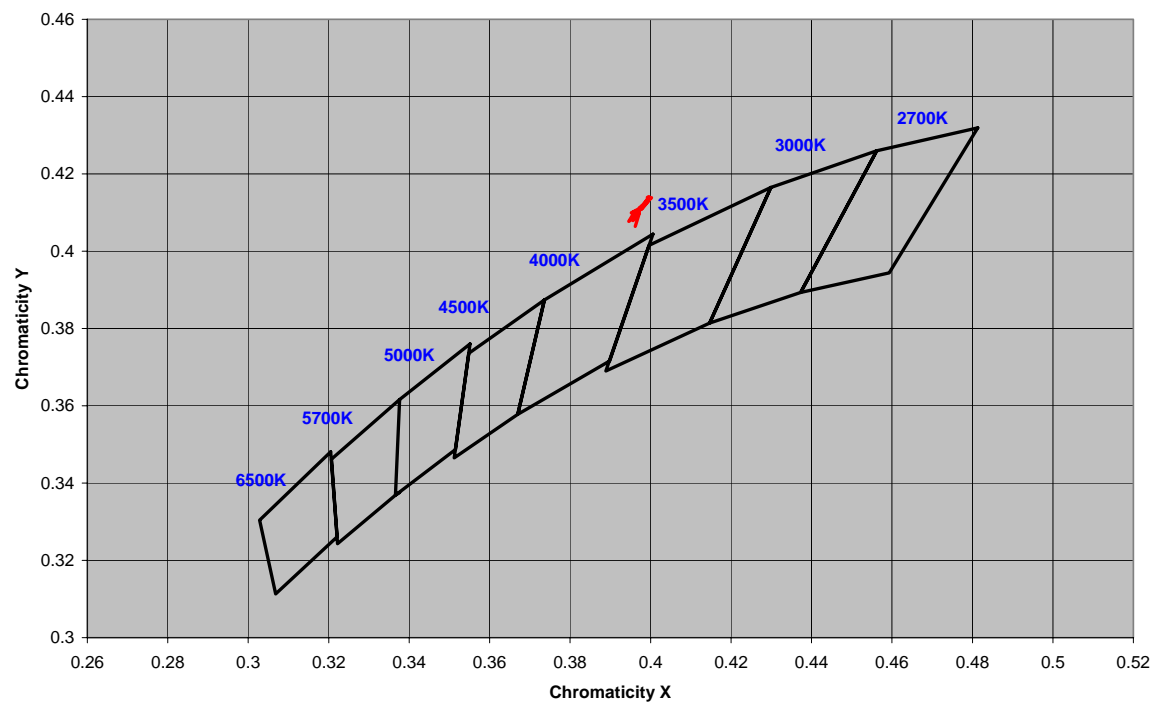

FLUOR-010D Chromacity Chart 


\begin{tabular}{|l|c|}
\hline Ilumisys Reference \# & INCAN-001D \\
\hline Manufacturer & GE \\
\hline Model & 16460 \\
\hline Description & GE CFL 13W Spiral Bulb \\
\hline $\begin{array}{l}\text { Power Supply } \\
\text { Voltage/Device }\end{array}$ & $420 \mathrm{Vac}$ \\
\hline $\begin{array}{l}\text { Ilumisys Initial Test } \\
\text { Date }\end{array}$ & $4 / 19 / 10$ \\
\hline $\begin{array}{l}\text { Ilumisys Final/ } \\
\text { Recent Test Date: }\end{array}$ & $8 / 19 / 11$ \\
\hline CALiPER Reference \# & $08-27$ \\
\hline CALiPER Test Round & 5 \\
\hline CALiPER Test Date & $4 / 08$ \\
\hline
\end{tabular}

\begin{tabular}{|l|c|c|c|}
\hline & $\begin{array}{c}\text { Manufacturer } \\
\text { Claim }\end{array}$ & $\begin{array}{c}\text { Test } \\
\text { Results }\end{array}$ & $\begin{array}{c}\text { CALiPER } \\
\text { Results }\end{array}$ \\
\hline $\begin{array}{l}\text { Initial Light Output } \\
\text { (Lumens) }\end{array}$ & 825 & 936 & 806 \\
\hline $\begin{array}{l}70 \% \text { Initial Output } \\
\text { (Lumens) }\end{array}$ & 578 & 655 & \\
\hline $\begin{array}{l}\text { Current Output } \\
\text { (Lumens) }\end{array}$ & & 681 & \\
\hline $\begin{array}{l}\text { Life Expectancy } \\
\text { (Hours) }\end{array}$ & 8,000 & 9,465 & 2,703 \\
\hline $\begin{array}{l}\text { Correlated Color } \\
\text { Temperature (K) }\end{array}$ & 2,700 & 2,695 & 82 \\
\hline $\begin{array}{l}\text { Color Rendering } \\
\text { Index (R9) }\end{array}$ & - & 81 & 12 \\
\hline Power (Watts) & 13 & 12 & \\
\hline
\end{tabular}

* Life expectancy for the test results is estimated using the exponential decay curve of the tested light output data and $70 \%$ of the initial output.

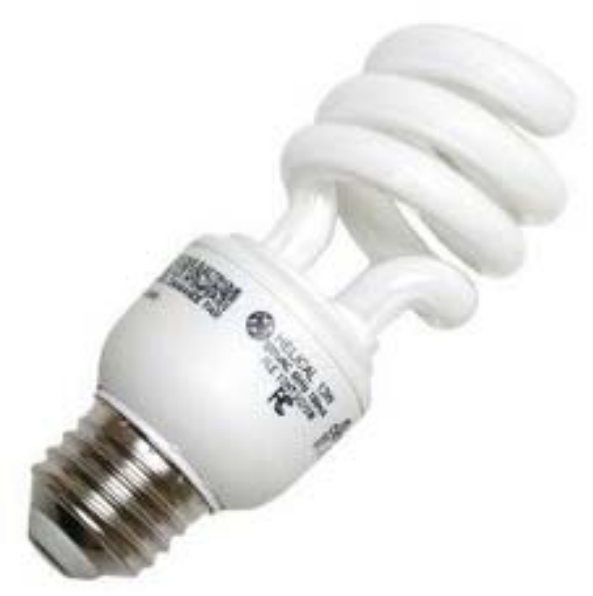




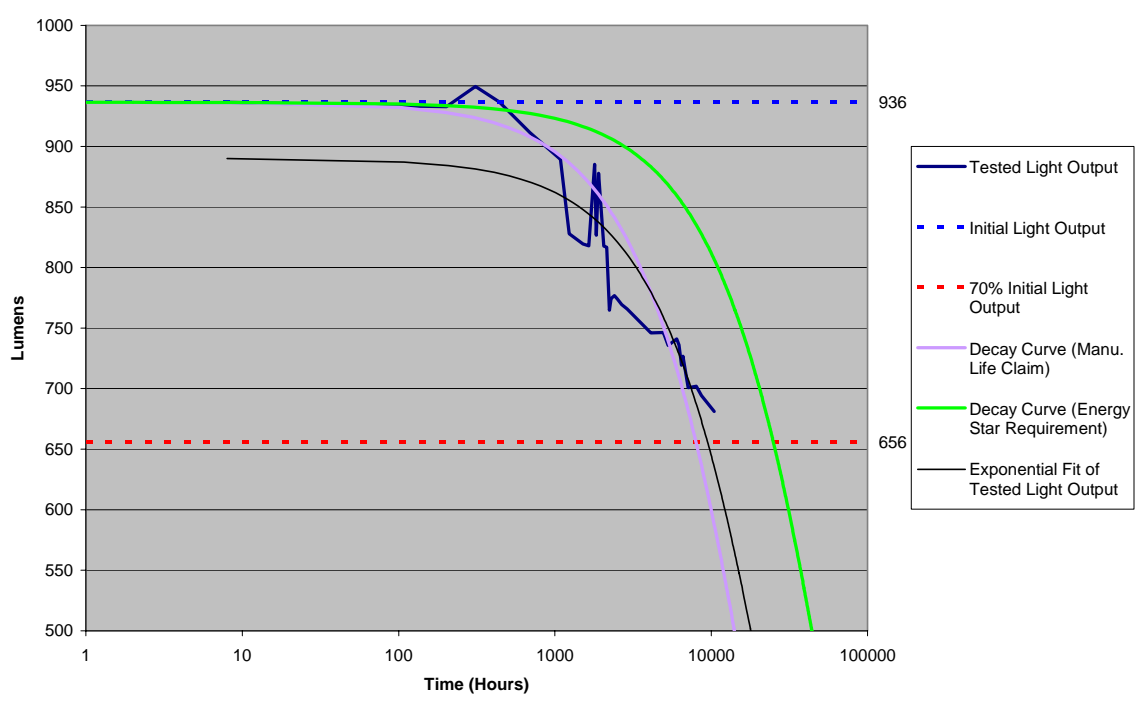

INCAN-001D Lumens vs. Time

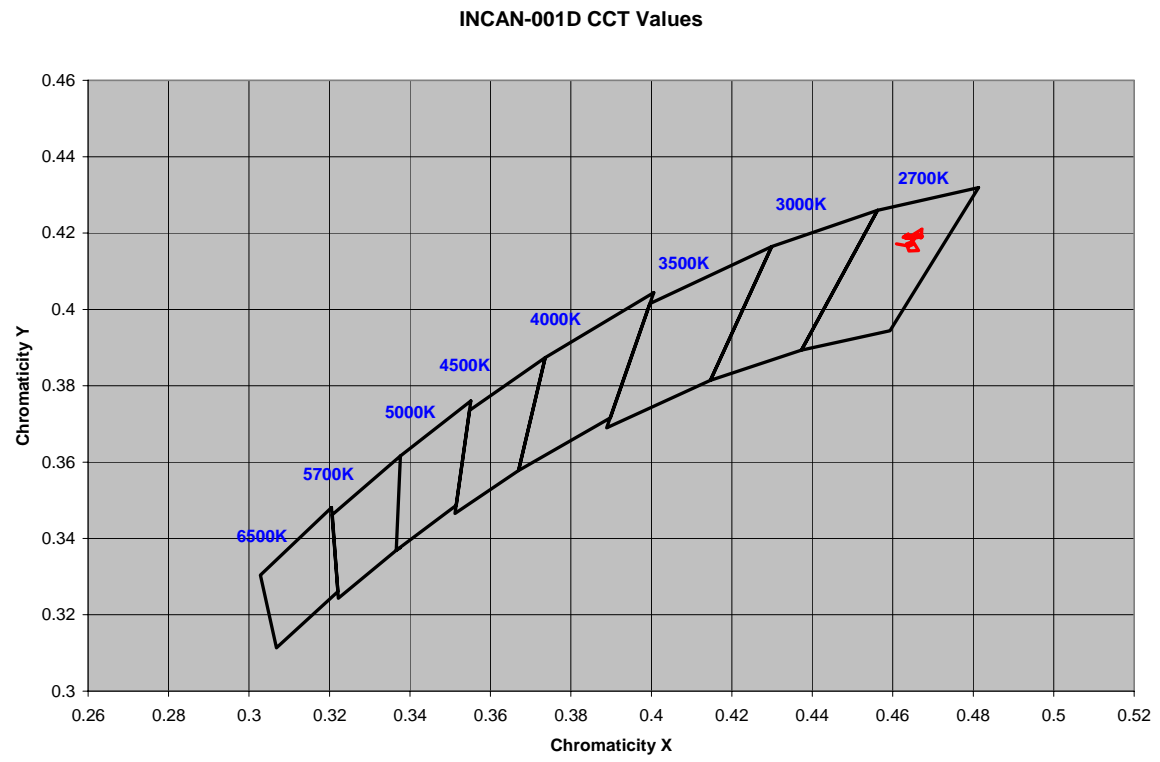

INCAN-001D Chromacity Chart 


\begin{tabular}{|l|c|}
\hline Ilumisys Reference \# & INCAN-002C \\
\hline Manufacturer & WDM \\
\hline Model & WDM 15W \\
\hline Description & Screw-in \\
\hline $\begin{array}{l}\text { Power Supply } \\
\text { Voltage/Device }\end{array}$ & $8 / 5 / 10$ \\
\hline $\begin{array}{l}\text { llumisys Initial Test } \\
\text { Date }\end{array}$ & $3 / 17 / 11$ \\
\hline $\begin{array}{l}\text { llumisys Final/ } \\
\text { Recent Test Date: }\end{array}$ & $08-132$ \\
\hline CALiPER Reference \# & 7 \\
\hline CALiPER Test Round & $12 / 1 / 08$ \\
\hline CALiPER Test Date & \\
\hline
\end{tabular}

\begin{tabular}{|l|c|c|c|}
\hline & $\begin{array}{c}\text { Manufacturer } \\
\text { Claim }\end{array}$ & $\begin{array}{c}\text { Test } \\
\text { Results }\end{array}$ & $\begin{array}{c}\text { CALiPER } \\
\text { Results }\end{array}$ \\
\hline $\begin{array}{l}\text { Initial Light Output } \\
\text { (Lumens) }\end{array}$ & 750 & 727 & 466 \\
\hline $\begin{array}{l}70 \% \text { Initial Output } \\
\text { (Lumens) }\end{array}$ & 525 & 509 & \\
\hline $\begin{array}{l}\text { Current Output } \\
\text { (Lumens) }\end{array}$ & 50,000 & $4,839 a$ & \\
\hline $\begin{array}{l}\text { Life Expectancy* } \\
\text { (Hours) }\end{array}$ & 3,500 & 5,483 & 3,743 \\
\hline $\begin{array}{l}\text { Correlated Color } \\
\text { Temperature (K) }\end{array}$ & - & 70 & 68 \\
\hline $\begin{array}{l}\text { Color Rendering } \\
\text { Index (R9) }\end{array}$ & 15 & 14 & 14 \\
\hline Power (Watts) & & & \\
\hline
\end{tabular}

* Life expectancy for the test results is estimated using the exponential decay curve of the tested light output data and $70 \%$ of the initial output.

a Device failed at this level many hours before testing was completed.

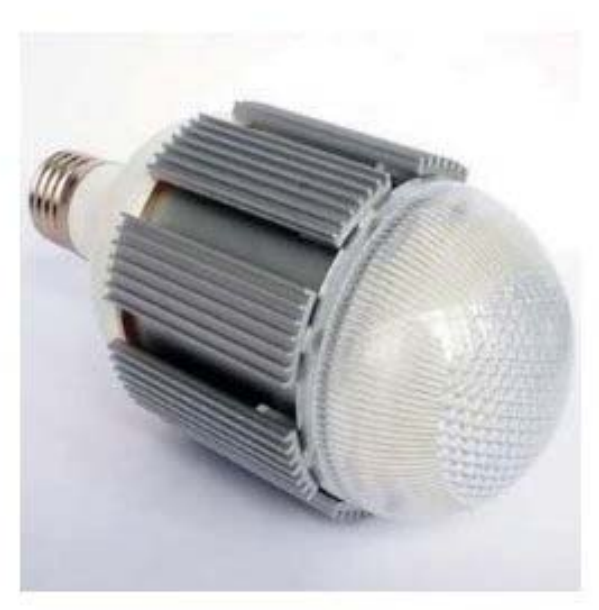




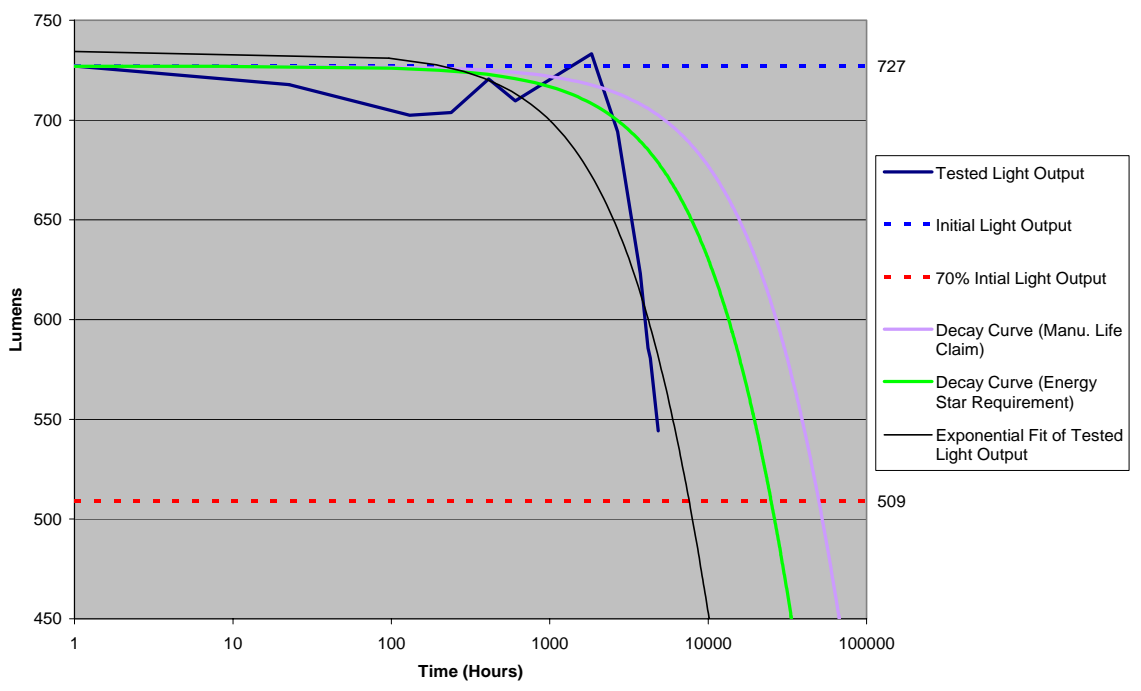

INCAN-002C Lumens vs. Time

CCT Values

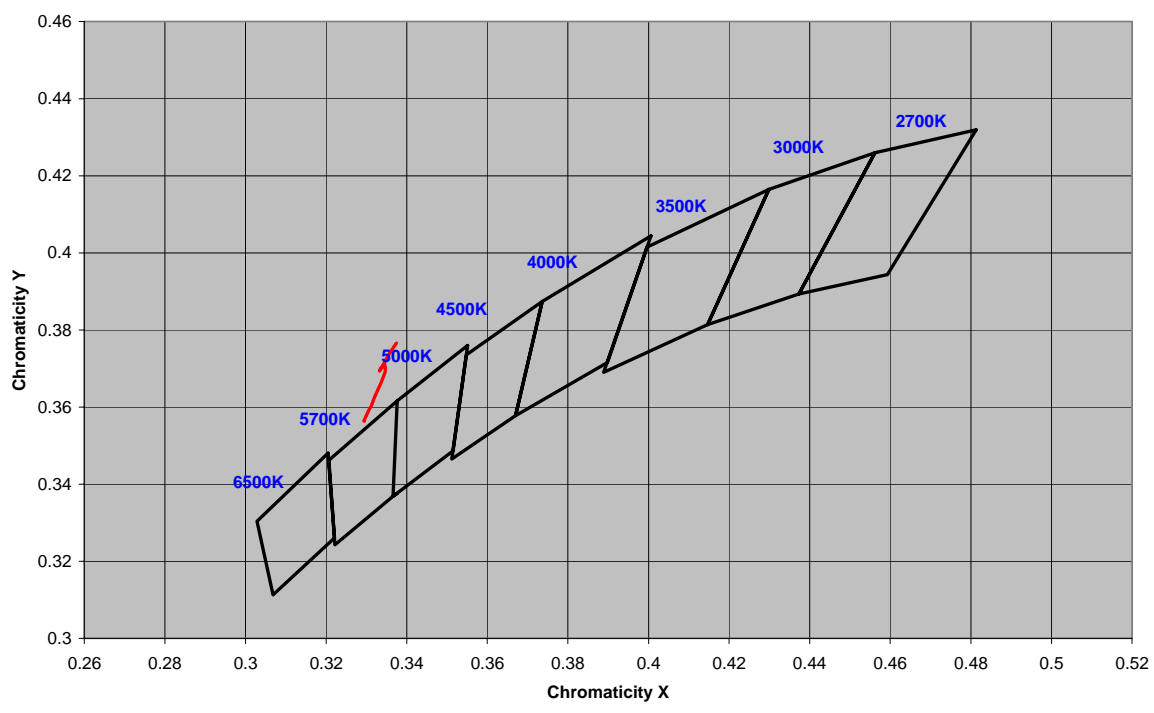

INCAN-002C Chromacity Chart 


\begin{tabular}{|l|c|}
\hline Ilumisys Reference \# & INCAN-005D \\
\hline Manufacturer & SLI \\
\hline Model & 120134 \\
\hline Description & Incandescent Long-Life 130V \\
\hline $\begin{array}{l}\text { Power Supply } \\
\text { Voltage/Device }\end{array}$ & $4 / 19 / 10$ \\
\hline $\begin{array}{l}\text { llumisys Initial Test } \\
\text { Date }\end{array}$ & $2 / 14 / 11$ \\
\hline $\begin{array}{l}\text { llumisys Final/ } \\
\text { Recent Test Date: }\end{array}$ & $08-04$ \\
\hline CALiPER Reference \# & 5 \\
\hline CALiPER Test Round & $4 / 08$ \\
\hline CALiPER Test Date & \\
\hline
\end{tabular}

\begin{tabular}{|l|c|c|c|}
\hline & $\begin{array}{c}\text { Manufacturer } \\
\text { Claim }\end{array}$ & $\begin{array}{c}\text { Test } \\
\text { Results }\end{array}$ & $\begin{array}{c}\text { CALiPER } \\
\text { Results }\end{array}$ \\
\hline $\begin{array}{l}\text { Initial Light Output } \\
\text { (Lumens) }\end{array}$ & 466 & 429 & 353 \\
\hline $\begin{array}{l}70 \% \text { Initial Output } \\
\text { (Lumens) }\end{array}$ & 325 & 300 & \\
\hline $\begin{array}{l}\text { Current Output } \\
\text { (Lumens) }\end{array}$ & $-5,000$ & 6,439 a & \\
\hline $\begin{array}{l}\text { Life Expectancy* } \\
\text { (Hours) }\end{array}$ & - & 2,565 & 2,491 \\
\hline $\begin{array}{l}\text { Correlated Color } \\
\text { Temperature (K) }\end{array}$ & - & 98 & 99 \\
\hline $\begin{array}{l}\text { Color Rendering } \\
\text { Index (R9) }\end{array}$ & 60 & 52 & 55 \\
\hline Power (Watts) & & & \\
\hline
\end{tabular}

* Life expectancy for the test results is estimated using the exponential decay curve of the tested light output data and $70 \%$ of the initial output.

a Device failed at this level many hours before testing was completed. 


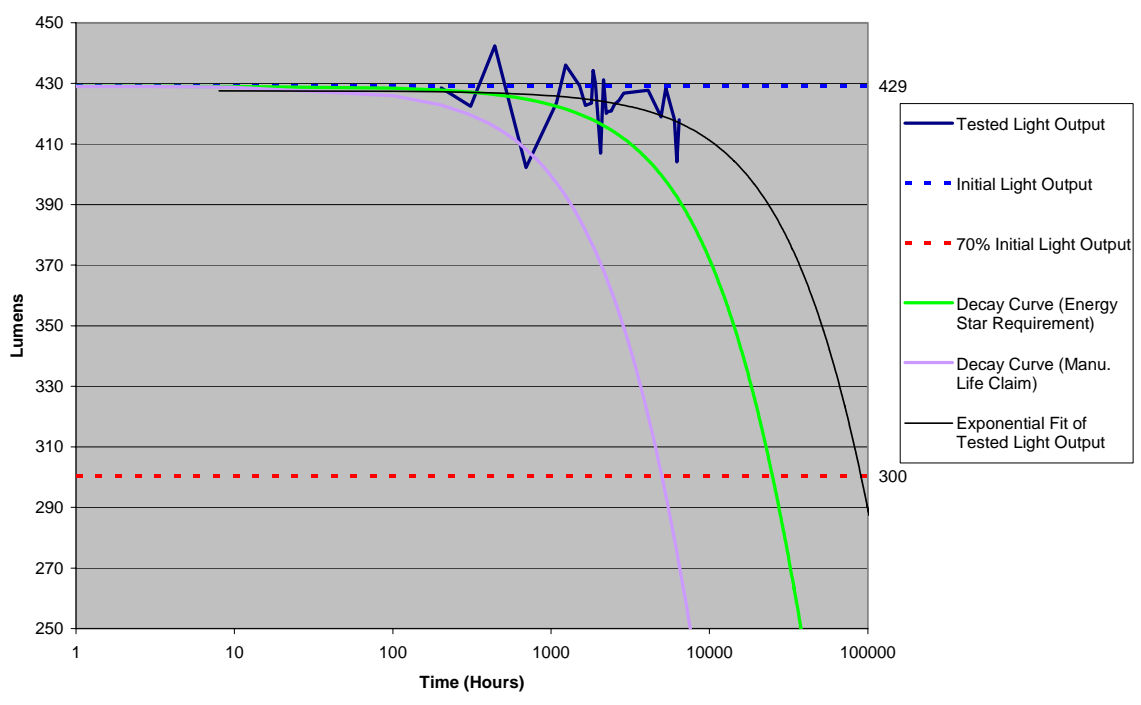

INCAN-005D Lumens vs. Time

CCT Values

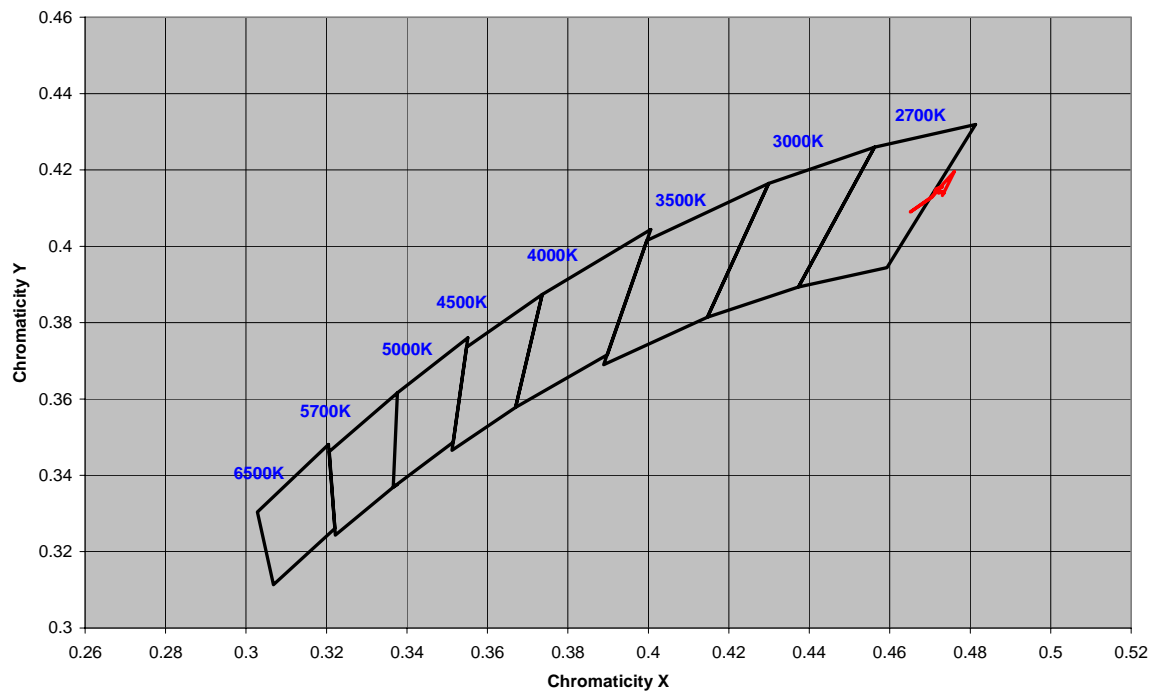

INCAN-005D Chromacity Chart 


\begin{tabular}{|l|c|}
\hline Ilumisys Reference \# & INCAN-006A \\
\hline Manufacturer & Mule Lighting \\
\hline Model & LEDalux LED-CW-120-CL \\
\hline Description & Decorative 1W LED Screw-in \\
\hline $\begin{array}{l}\text { Power Supply } \\
\text { Voltage/Device }\end{array}$ & $4 / 18 / 10$ \\
\hline $\begin{array}{l}\text { Ilumisys Initial Test } \\
\text { Date }\end{array}$ & $8 / 17 / 11$ \\
\hline $\begin{array}{l}\text { Ilumisys Final/ } \\
\text { Recent Test Date: }\end{array}$ & $07 / 12$ \\
\hline CALiPER Reference \# & 2 \\
\hline CALiPER Test Round & $5 / 16 / 07$ \\
\hline CALiPER Test Date & \\
\hline
\end{tabular}

\begin{tabular}{|l|c|c|c|}
\hline & $\begin{array}{c}\text { Manufacturer } \\
\text { Claim }\end{array}$ & $\begin{array}{c}\text { Test } \\
\text { Results }\end{array}$ & $\begin{array}{c}\text { CALiPER } \\
\text { Results }\end{array}$ \\
\hline $\begin{array}{l}\text { Initial Light Output } \\
\text { (Lumens) }\end{array}$ & - & 11 & 20 \\
\hline $\begin{array}{l}\text { 70\% Initial Output } \\
\text { (Lumens) }\end{array}$ & - & 8 & \\
\hline $\begin{array}{l}\text { Current Output } \\
\text { (Lumens) }\end{array}$ & & 4 & \\
\hline $\begin{array}{l}\text { Life Expectancy* } \\
\text { (Hours) }\end{array}$ & 50,000 & $6103 \mathrm{a}$ & \\
\hline $\begin{array}{l}\text { Correlated Color } \\
\text { Temperature (K) }\end{array}$ & - & 13,073 & 25,263 \\
\hline $\begin{array}{l}\text { Color Rendering } \\
\text { Index (R9) }\end{array}$ & - & 79 & 79 \\
\hline Power (Watts) & $<1$ & 1 & 1.5 \\
\hline
\end{tabular}

* Life expectancy for the test results is estimated using the exponential decay curve of the tested light output data and $70 \%$ of the initial output.

a It is estimated the device performance fell below $70 \%$ initial output at this level for many hours.

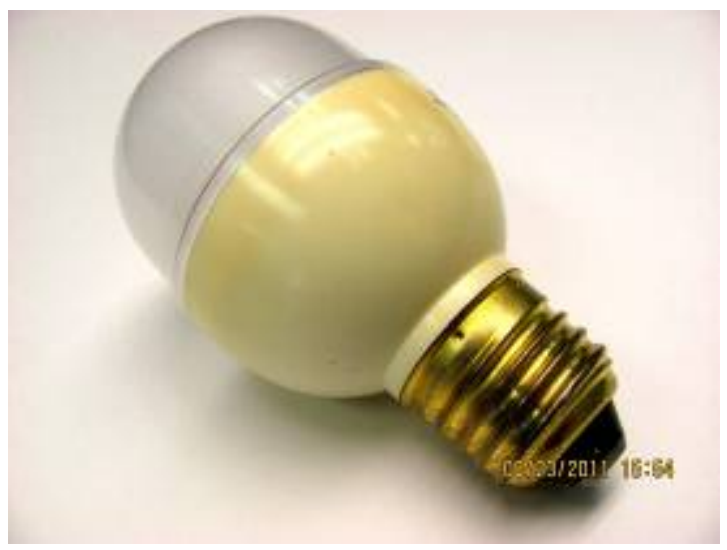




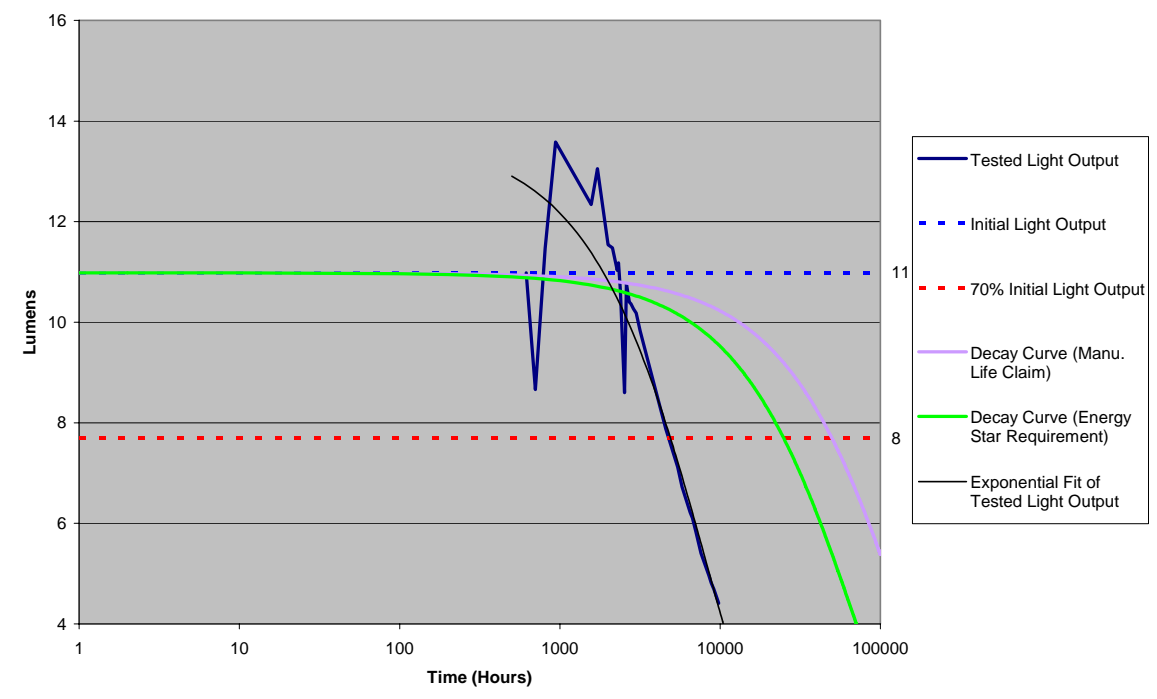

INCAN-006A Lumens vs. Time

CCT Values

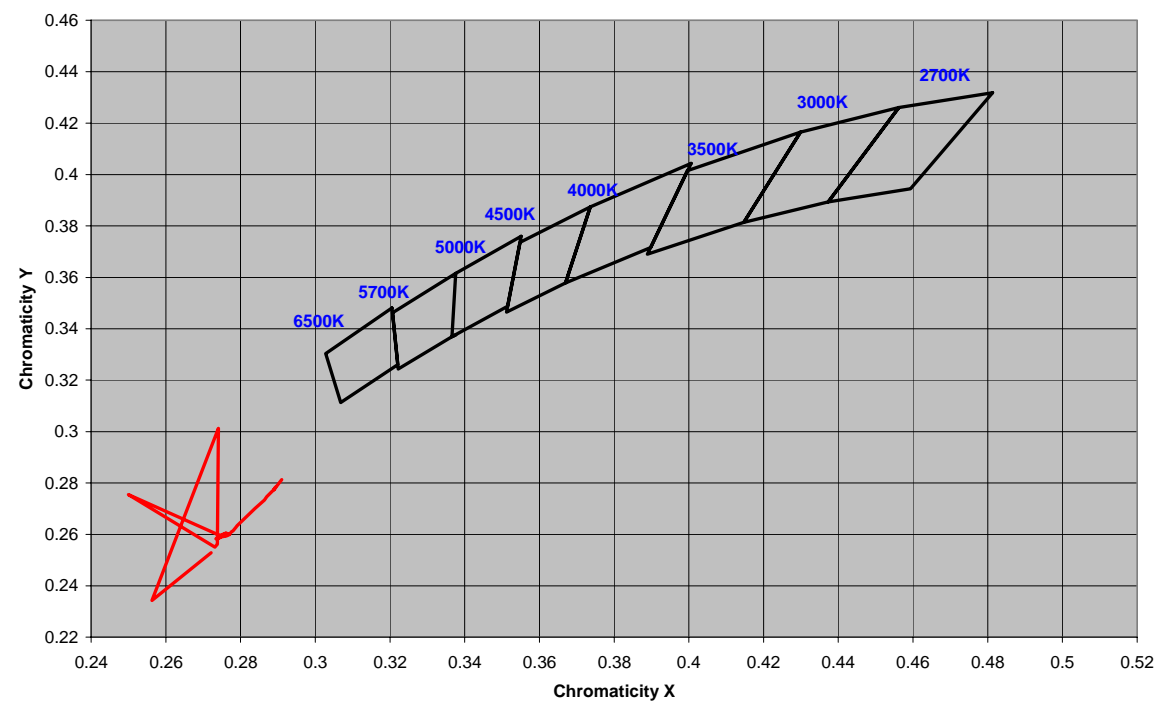

INCAN-006A Chromacity Chart 


\begin{tabular}{|l|c|}
\hline Ilumisys Reference \# & INCAN-008D \\
\hline Manufacturer & GBL LED Lighting \\
\hline Model & 150 LED E26-D5X30LED-CLR \\
\hline Description & 150 LED Screw-in Bulb \\
\hline $\begin{array}{l}\text { Power Supply } \\
\text { Voltage/Device }\end{array}$ & $4 / 20 / 10$ \\
\hline $\begin{array}{l}\text { Ilumisys Initial Test } \\
\text { Date }\end{array}$ & $8 / 17 / 11$ \\
\hline $\begin{array}{l}\text { Ilumisys Final/ } \\
\text { Recent Test Date: }\end{array}$ & $08 / 80$ \\
\hline CALiPER Reference \# & 6 \\
\hline CALiPER Test Round & $8 / 08$ \\
\hline CALiPER Test Date & \\
\hline
\end{tabular}

\begin{tabular}{|l|c|c|c|}
\hline & $\begin{array}{c}\text { Manufacturer } \\
\text { Claim }\end{array}$ & $\begin{array}{c}\text { Test } \\
\text { Results }\end{array}$ & $\begin{array}{c}\text { CALiPER } \\
\text { Results }\end{array}$ \\
\hline $\begin{array}{l}\text { Initial Light Output } \\
\text { (Lumens) }\end{array}$ & 295 & 193 & 292 \\
\hline $\begin{array}{l}\text { 70\% Initial Output } \\
\text { (Lumens) }\end{array}$ & 206.5 & 135 & \\
\hline $\begin{array}{l}\text { Current Output } \\
\text { (Lumens) }\end{array}$ & & 16 & \\
\hline $\begin{array}{l}\text { Life Expectancy } \\
\text { (Hours) }\end{array}$ & 50,000 & $513 a$ & 7,272 \\
\hline $\begin{array}{l}\text { Correlated Color } \\
\text { Temperature (K) }\end{array}$ & - & 9,655 & 79 \\
\hline $\begin{array}{l}\text { Color Rendering } \\
\text { Index (R9) }\end{array}$ & - & 82 & 4.8 \\
\hline Power (Watts) & - & 4 & \\
\hline
\end{tabular}

* Life expectancy for the test results is estimated using the exponential decay curve of the tested light output data and $70 \%$ of the initial output.

a It is estimated the device performance fell below $70 \%$ initial output at this level for many hours.

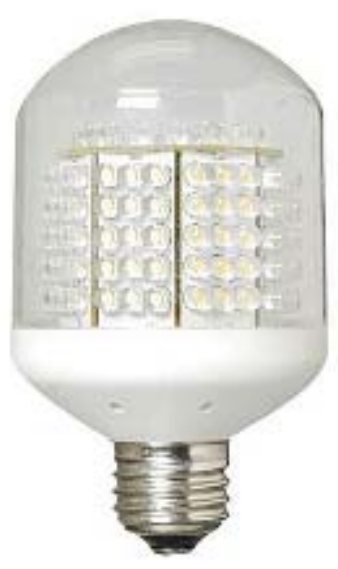




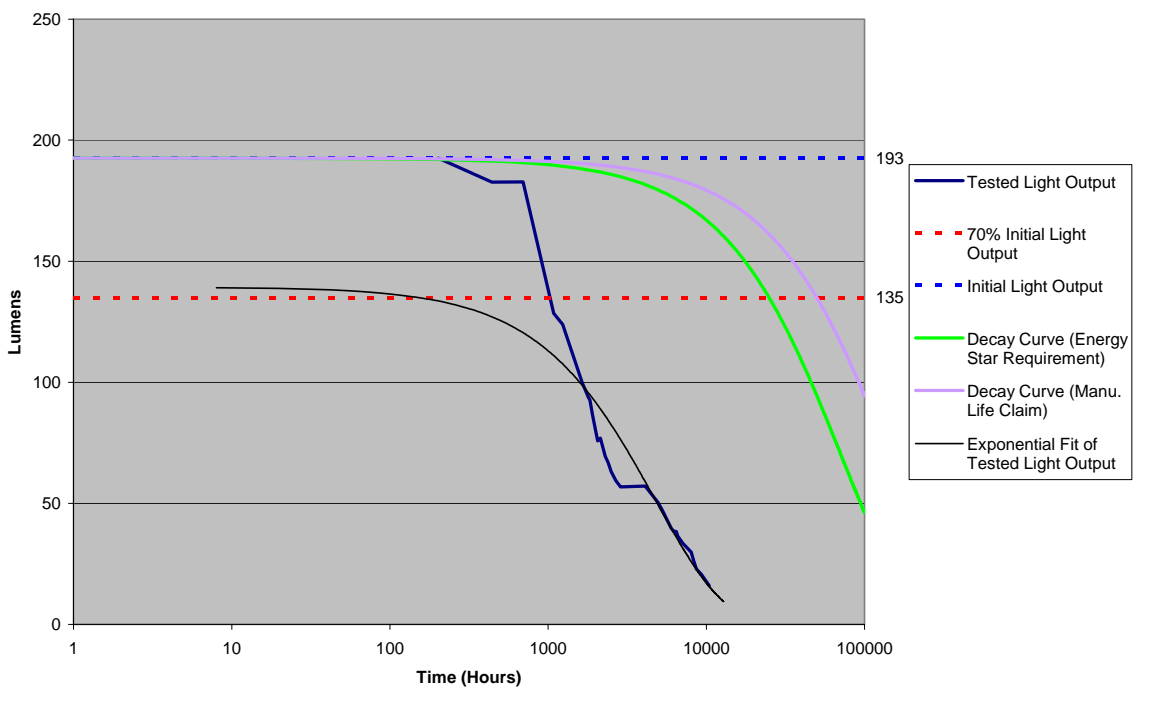

INCAN-008D Lumens vs. Time

CCT Values

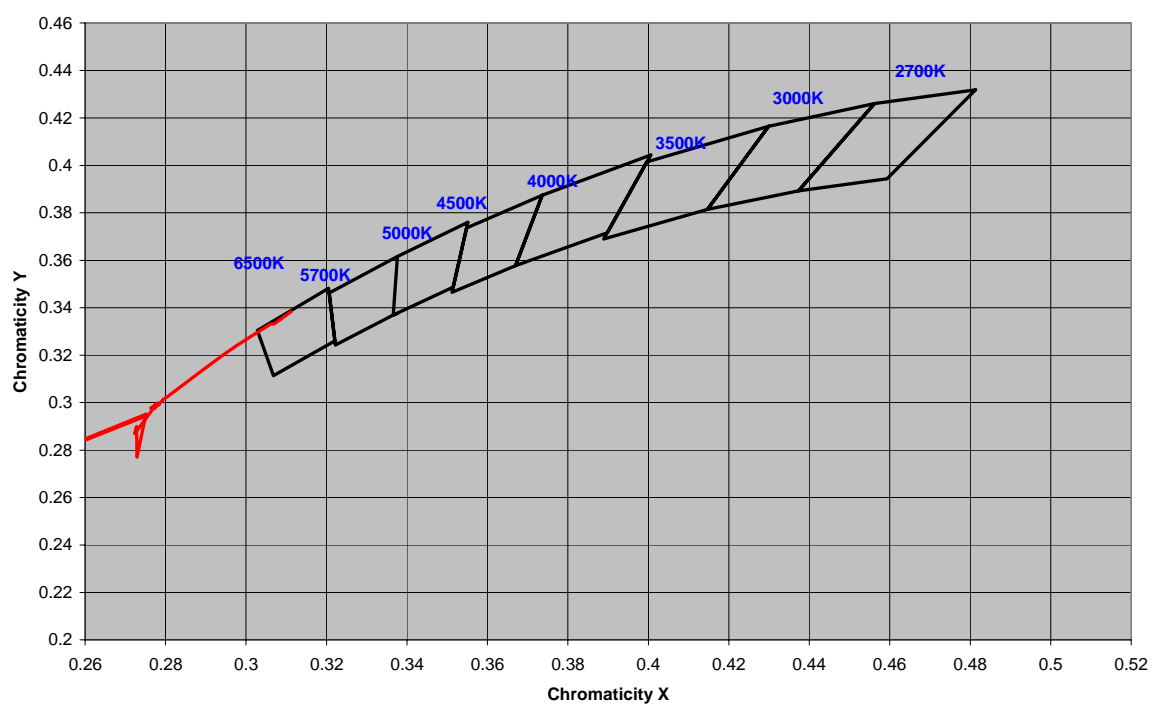

INCAN-008D Chromacity Chart 


\begin{tabular}{|c|c|}
\hline Ilumisys Reference \# & INCAN-015B \\
\hline Manufacturer & Sylvania \\
\hline Model & 78779 \\
\hline Description & $\begin{array}{l}\text { 8W LED Screw-in } \\
\text { (40W Replacement) }\end{array}$ \\
\hline $\begin{array}{l}\text { Power Supply } \\
\text { Voltage/Device }\end{array}$ & 120Vac \\
\hline $\begin{array}{l}\text { llumisys Initial Test } \\
\text { Date }\end{array}$ & $4 / 26 / 10$ \\
\hline $\begin{array}{l}\text { Ilumisys Final/ } \\
\text { Recent Test Date: }\end{array}$ & $8 / 17 / 11$ \\
\hline CALiPER Reference \# & - \\
\hline CALiPER Test Round & - \\
\hline CALiPER Test Date & - \\
\hline
\end{tabular}

\begin{tabular}{|l|c|c|c|}
\hline & $\begin{array}{c}\text { Manufacturer } \\
\text { Claim }\end{array}$ & $\begin{array}{c}\text { Test } \\
\text { Results }\end{array}$ & $\begin{array}{c}\text { CALiPER } \\
\text { Results }\end{array}$ \\
\hline $\begin{array}{l}\text { Initial Light Output } \\
\text { (Lumens) }\end{array}$ & 390 & 369 & - \\
\hline $\begin{array}{l}70 \% \text { Initial Output } \\
\text { (Lumens) }\end{array}$ & 273 & 258 & \\
\hline $\begin{array}{l}\text { Current Output } \\
\text { (Lumens) }\end{array}$ & & 323 & \\
\hline $\begin{array}{l}\text { Life Expectancy* } \\
\text { (Hours) }\end{array}$ & 50,000 & 43,659 & \\
\hline $\begin{array}{l}\text { Correlated Color } \\
\text { Temperature (K) }\end{array}$ & 3,000 & 3,396 & - \\
\hline $\begin{array}{l}\text { Color Rendering } \\
\text { Index (R9) }\end{array}$ & 85 & 83 & - \\
\hline Power (Watts) & 8 & 8 & - \\
\hline
\end{tabular}

* Life expectancy for the test results is estimated using the exponential decay curve of the tested light output data and $70 \%$ of the initial output.

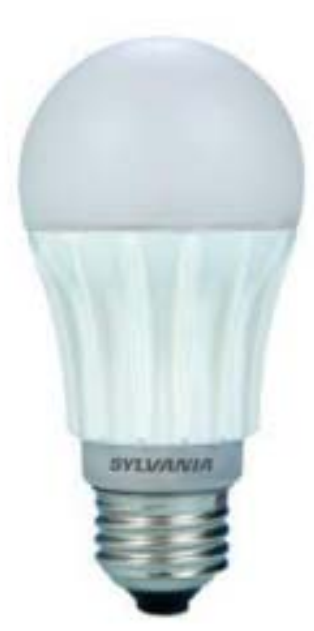




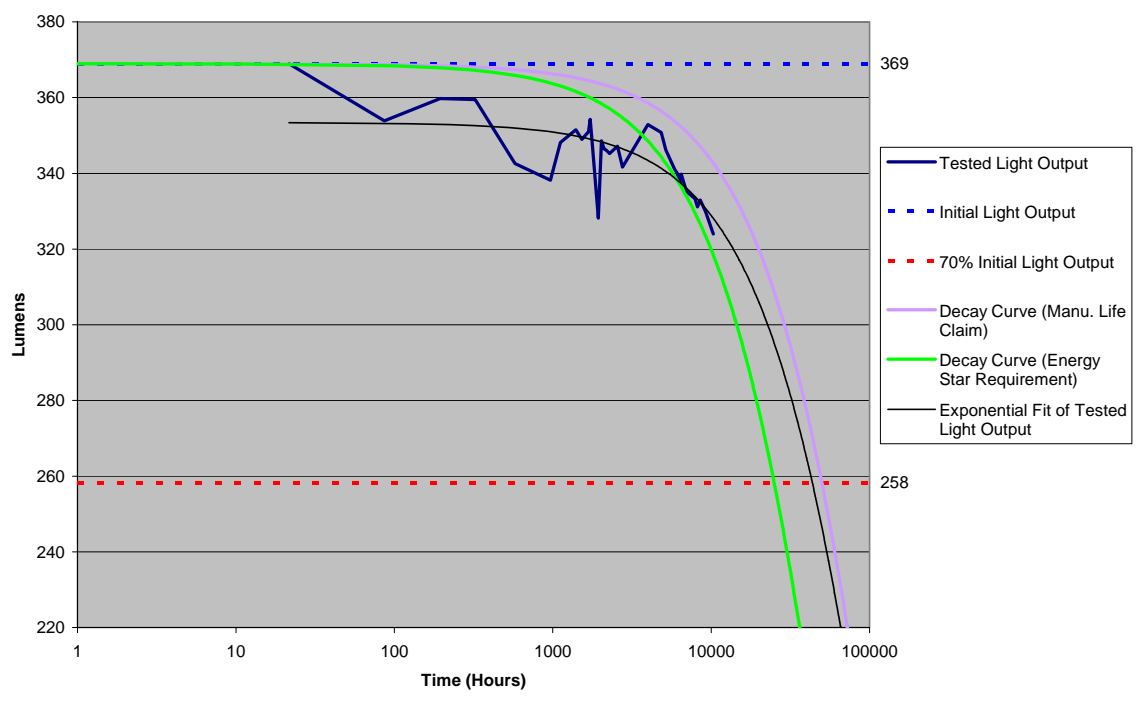

INCAN-015B Lumens vs. Time

CCT Values

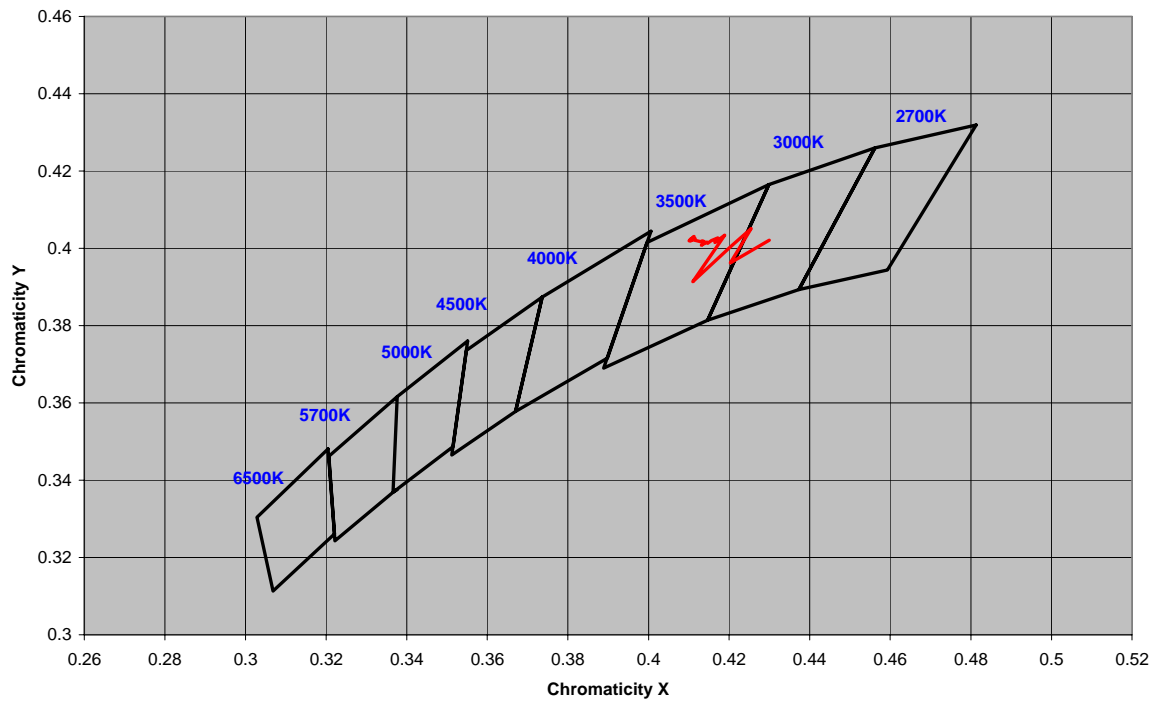

INCAN-015B Chromacity Chart 


\begin{tabular}{|l|c|}
\hline Ilumisys Reference \# & INCAN-017A \\
\hline Manufacturer & Philips \\
\hline Model & Ambient LED Indoor Bulb \\
\hline Description & 1208369 \\
\hline $\begin{array}{l}\text { Power Supply } \\
\text { Voltage/Device }\end{array}$ & $8 / 5 / 10$ \\
\hline $\begin{array}{l}\text { llumisys Initial Test } \\
\text { Date }\end{array}$ & $8 / 17 / 11$ \\
\hline $\begin{array}{l}\text { llumisys Final/ } \\
\text { Recent Test Date: }\end{array}$ & - \\
\hline CALiPER Reference \# & - \\
\hline CALiPER Test Round & - \\
\hline CALiPER Test Date & \\
\hline
\end{tabular}

\begin{tabular}{|l|c|c|c|}
\hline & $\begin{array}{c}\text { Manufacturer } \\
\text { Claim }\end{array}$ & $\begin{array}{c}\text { Test } \\
\text { Results }\end{array}$ & $\begin{array}{c}\text { CALiPER } \\
\text { Results }\end{array}$ \\
\hline $\begin{array}{l}\text { Initial Light Output } \\
\text { (Lumens) }\end{array}$ & 240 & 286 & - \\
\hline $\begin{array}{l}70 \% \text { Initial Output } \\
\text { (Lumens) }\end{array}$ & 168 & 200 & \\
\hline $\begin{array}{l}\text { Current Output } \\
\text { (Lumens) }\end{array}$ & 25,000 & 37,831 & \\
\hline $\begin{array}{l}\text { Life Expectancy* } \\
\text { (Hours) }\end{array}$ & 3,000 & 2,969 & - \\
\hline $\begin{array}{l}\text { Correlated Color } \\
\text { Temperature (K) }\end{array}$ & - & 83 & - \\
\hline $\begin{array}{l}\text { Color Rendering } \\
\text { Index (Rg) }\end{array}$ & 5 & 5 & - \\
\hline Power (Watts) & & & - \\
\hline
\end{tabular}

* Life expectancy for the test results is estimated using the exponential decay curve of the tested light output data and $70 \%$ of the initial output.

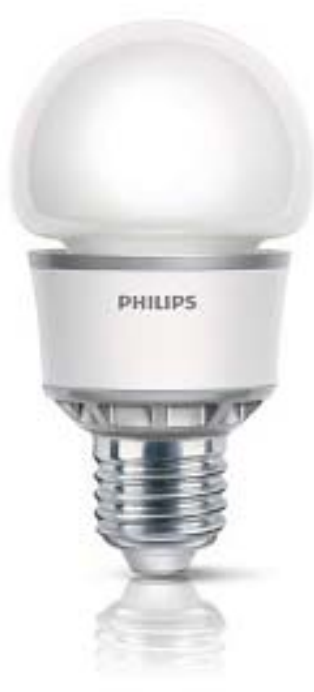




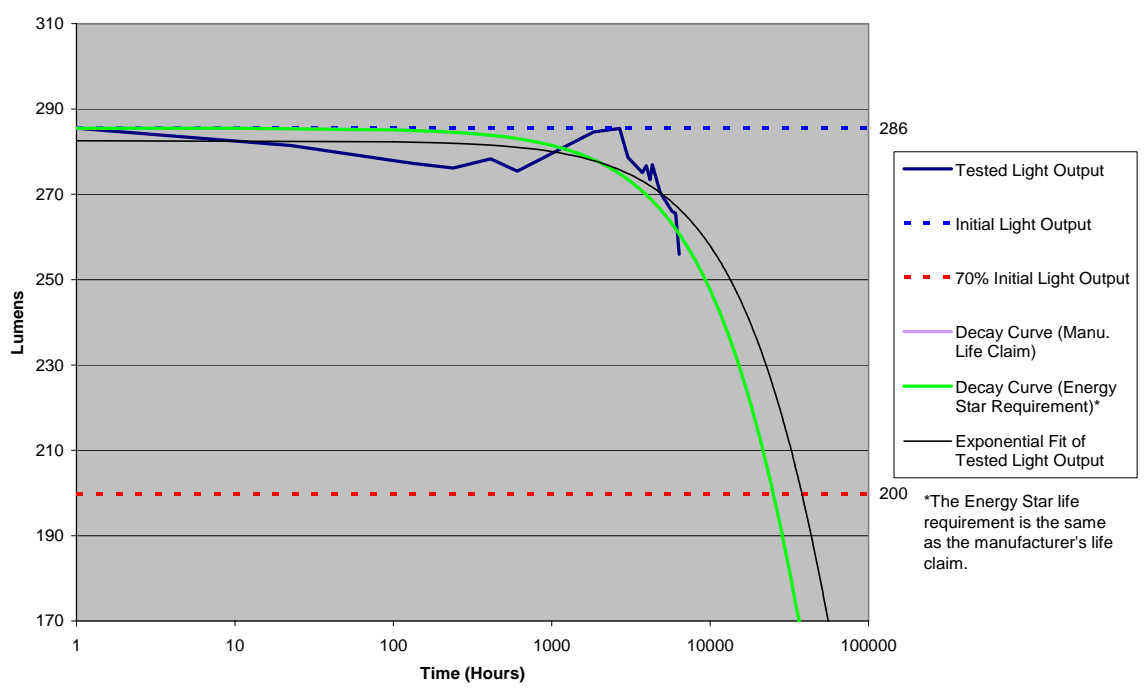

INCAN-017A Lumens vs. Time

CCT Values

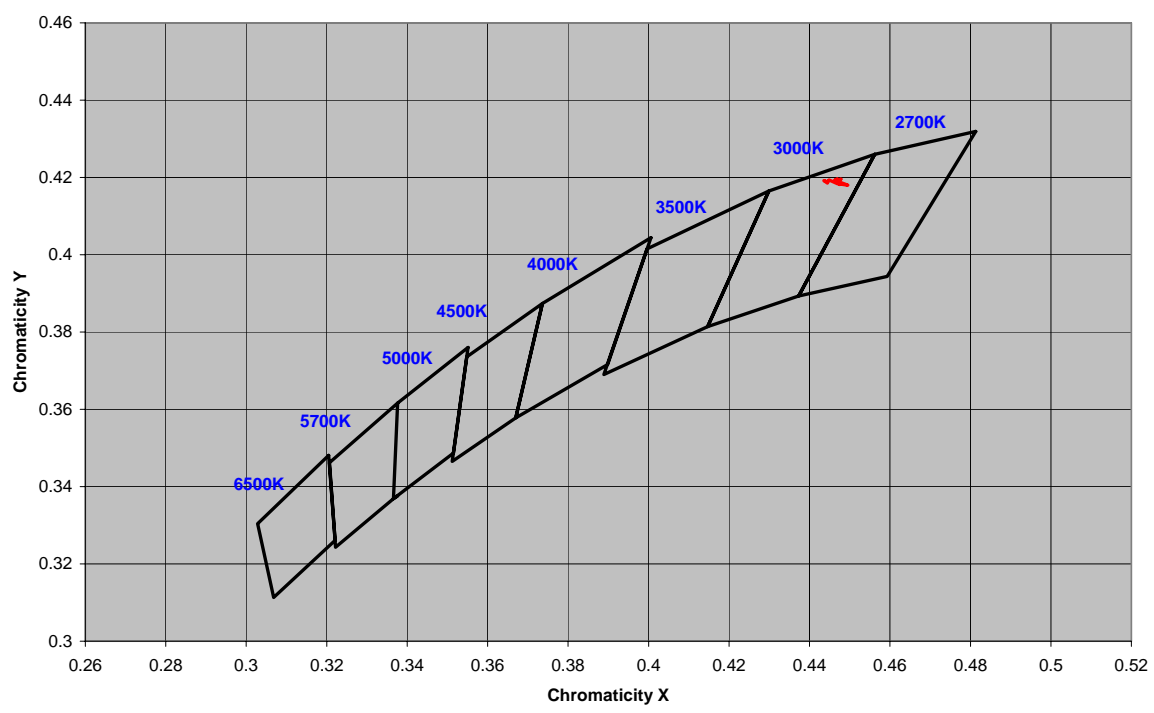

INCAN-017A Chromacity Chart 


\begin{tabular}{|l|c|}
\hline Ilumisys Reference \# & INCAN-007B \\
\hline Manufacturer & Earth LED \\
\hline Model & Warm White LED Screw-in \\
\hline Description & 120 Vac \\
\hline $\begin{array}{l}\text { Power Supply } \\
\text { Voltage/Device }\end{array}$ & $4 / 18 / 10$ \\
\hline $\begin{array}{l}\text { llumisys Initial Test } \\
\text { Date }\end{array}$ & $8 / 17 / 11$ \\
\hline $\begin{array}{l}\text { llumisys Final/ } \\
\text { Recent Test Date: }\end{array}$ & $08-92$ \\
\hline CALiPER Reference \# & 6 \\
\hline CALiPER Test Round & $8 / 08$ \\
\hline CALiPER Test Date & \\
\hline
\end{tabular}

\begin{tabular}{|l|c|c|c|}
\hline & $\begin{array}{c}\text { Manufacturer } \\
\text { Claim }\end{array}$ & $\begin{array}{c}\text { Test } \\
\text { Results }\end{array}$ & $\begin{array}{c}\text { CALiPER } \\
\text { Results }\end{array}$ \\
\hline $\begin{array}{l}\text { Initial Light Output } \\
\text { (Lumens) }\end{array}$ & 500 & 392 & 403 \\
\hline $\begin{array}{l}70 \% \text { Initial Output } \\
\text { (Lumens) }\end{array}$ & 350 & 274 & \\
\hline $\begin{array}{l}\text { Current Output } \\
\text { (Lumens) }\end{array}$ & 50,000 & 42,679 & \\
\hline $\begin{array}{l}\text { Life Expectancy } \\
\text { (Hours) }\end{array}$ & 3,000 & 2,906 & 3,143 \\
\hline $\begin{array}{l}\text { Correlated Color } \\
\text { Temperature (K) }\end{array}$ & 80 & 83 & 49 \\
\hline $\begin{array}{l}\text { Color Rendering } \\
\text { Index (R9) }\end{array}$ & 13 & 12 & 12.9 \\
\hline Power (Watts) & & & \\
\hline
\end{tabular}

* Life expectancy for the test results is estimated using the exponential decay curve of the tested light output data and $70 \%$ of the initial output.

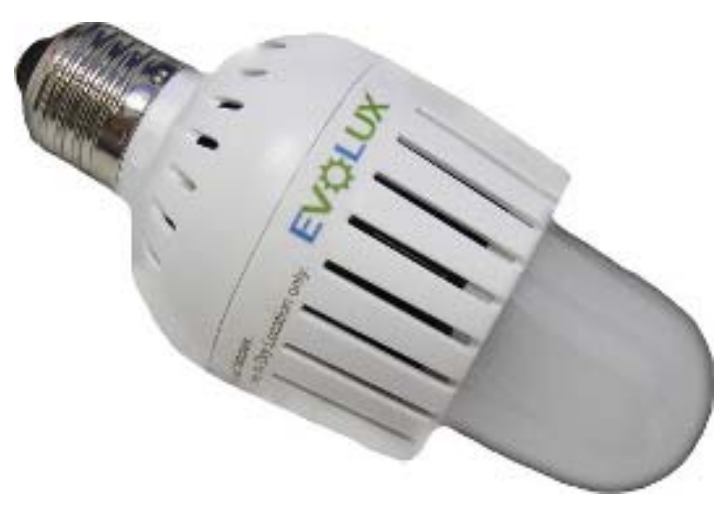




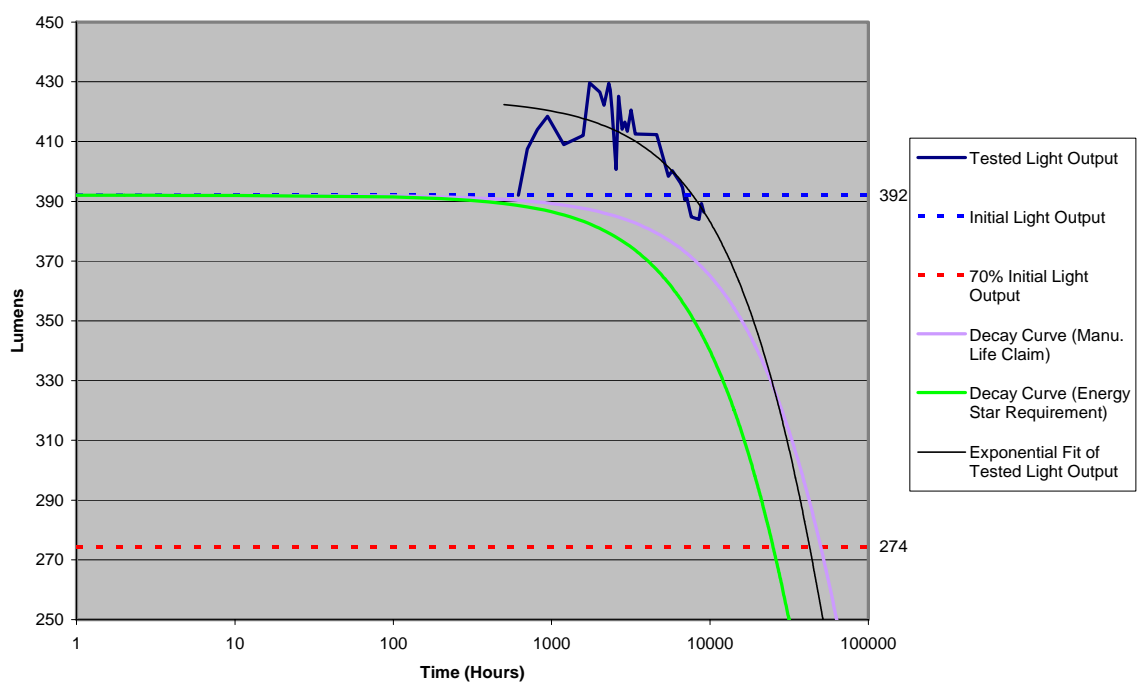

INCAN-007B Lumens vs. Time

CCT Values

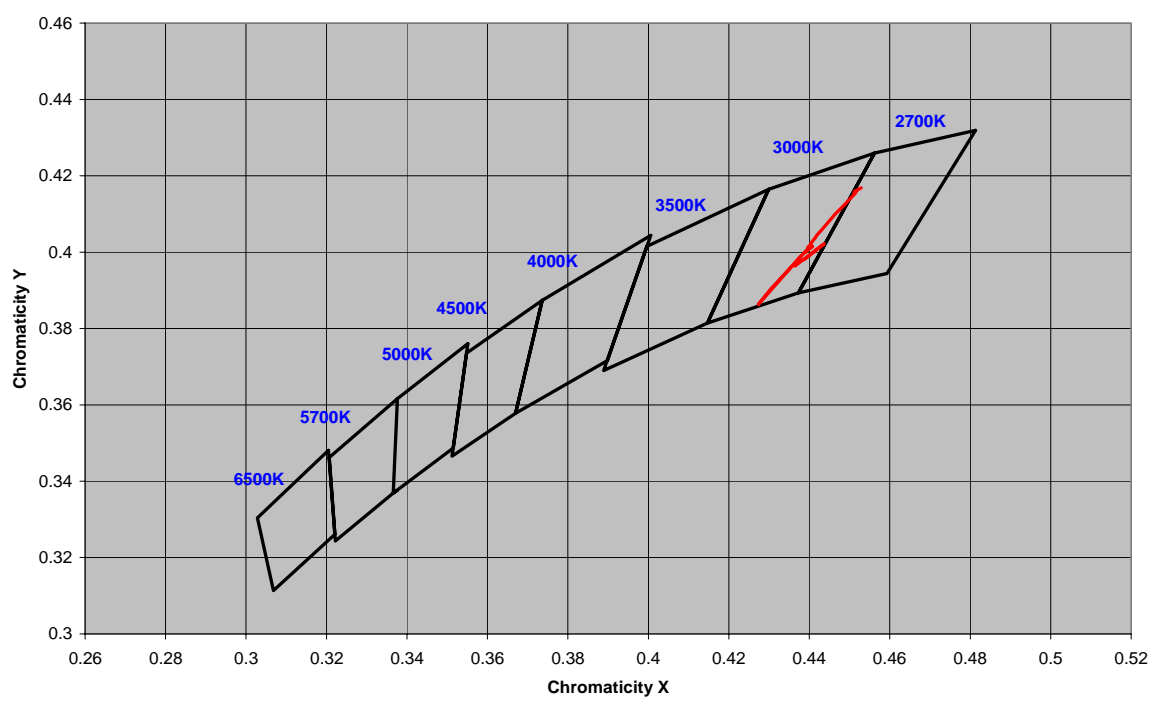

INCAN-007B Chromacity Chart 


\begin{tabular}{|l|c|}
\hline llumisys Reference \# & INCAN-018B \\
\hline Manufacturer & GE \\
\hline Model & 13196 Spiral CFL \\
\hline Description & 120Vac \\
\hline $\begin{array}{l}\text { Power Supply } \\
\text { Voltage/Device }\end{array}$ & $8 / 18 / 10$ \\
\hline $\begin{array}{l}\text { llumisys Initial Test } \\
\text { Date }\end{array}$ & $8 / 17 / 11$ \\
\hline $\begin{array}{l}\text { llumisys Final/ } \\
\text { Recent Test Date: }\end{array}$ & - \\
\hline CALiPER Reference \# & - \\
\hline CALiPER Test Round & - \\
\hline CALiPER Test Date & \\
\hline
\end{tabular}

\begin{tabular}{|l|c|c|c|}
\hline & $\begin{array}{c}\text { Manufacturer } \\
\text { Claim }\end{array}$ & $\begin{array}{c}\text { Test } \\
\text { Results }\end{array}$ & $\begin{array}{c}\text { CALiPER } \\
\text { Results }\end{array}$ \\
\hline $\begin{array}{l}\text { Initial Light Output } \\
\text { (Lumens) }\end{array}$ & 825 & 842 & - \\
\hline $\begin{array}{l}\text { 70\% Initial Output } \\
\text { (Lumens) }\end{array}$ & 578 & 589 & \\
\hline $\begin{array}{l}\text { Current Output } \\
\text { (Lumens) }\end{array}$ & & 613 & \\
\hline $\begin{array}{l}\text { Life Expectancy } \\
\text { (Hours) }\end{array}$ & 8,000 & 7,003 & - \\
\hline $\begin{array}{l}\text { Correlated Color } \\
\text { Temperature (K) }\end{array}$ & 2,700 & 2,748 & - \\
\hline $\begin{array}{l}\text { Color Rendering } \\
\text { Index (R9) }\end{array}$ & 82 & 81 & - \\
\hline Power (Watts) & 13 & 11 & - \\
\hline
\end{tabular}

* Life expectancy for the test results is estimated using the exponential decay curve of the tested light output data and $70 \%$ of the initial output.

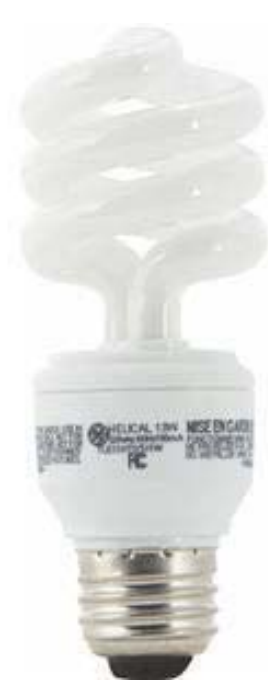




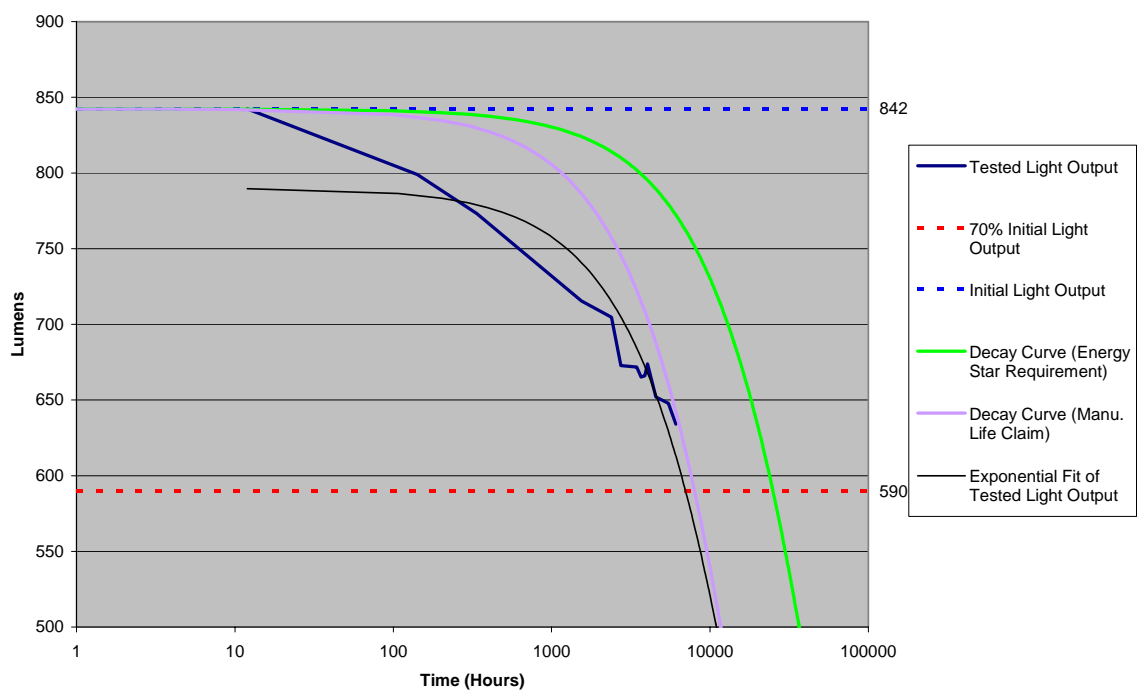

INCAN-018B Lumens vs. Time

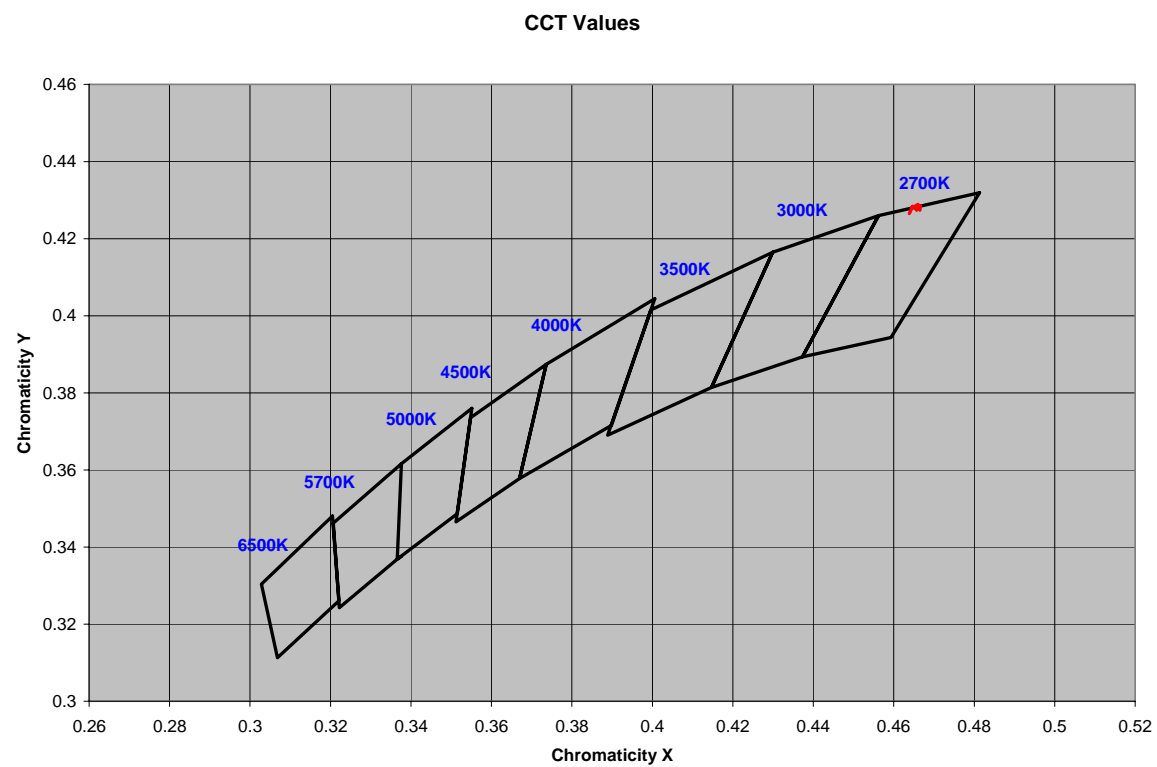

INCAN-018B Chromacity Chart 


\section{Appendix B - Environmental Impact Values and Documentation}

\section{Impact Values}

Below are the environmental values used in the Sustainable LED Design Guide.

\begin{tabular}{|c|c|c|c|c|c|c|c|}
\hline $\begin{array}{c}\text { Material } \\
\text { Class } \\
\end{array}$ & Material & Primary Process & $\begin{array}{c}\text { Secondary } \\
\text { Process }\end{array}$ & $\begin{array}{c}\text { Process } \\
\text { Measurable }\end{array}$ & $\begin{array}{c}\mathrm{GHG} \\
\left(\mathrm{kg} \mathrm{CO} \mathrm{CO}_{2} \mathrm{Eq}\right)\end{array}$ & $\begin{array}{c}\text { Energy } \\
\text { (MJ) }\end{array}$ & $\begin{array}{l}\text { Mercury } \\
\text { (mg) }\end{array}$ \\
\hline Ferro & Steel & Steel Billet & None & Weight & 1.815667096 & 28.22468773 & 0.635260656 \\
\hline Ferro & Steel & Steel Billet & Wire Drawing & Weight & 2.147002536 & 32.37091735 & 0.983572346 \\
\hline Ferro & Steel & Steel Billet & Milling & Weight & 3.080300952 & 46.90177909 & 1.051343551 \\
\hline Ferro & Steel & Steel Billet & Chrome Finish & Surface Area & 2.1711783 & 43.4658086 & 0.15263142 \\
\hline Ferro & Steel & Steel Billet & Powder Coating & Surface Area & 4.589747 & 86.1691345 & 0.44941253 \\
\hline Ferro & Steel & $\begin{array}{c}\text { Stamped Primary } \\
\text { Sheet }\end{array}$ & None & Weight & 3.007681413 & 37.98300005 & 0.797583869 \\
\hline Ferro & Steel & $\begin{array}{c}\text { Stamped Primary } \\
\text { Sheet }\end{array}$ & Chrome Finish & Surface Area & 2.1711783 & 43.4658086 & 0.15263142 \\
\hline Ferro & Steel & $\begin{array}{c}\text { Stamped Primary } \\
\text { Sheet }\end{array}$ & Powder Coating & Surface Area & 4.589747 & 86.1691345 & 0.44941253 \\
\hline Ferro & Steel & $\begin{array}{c}\text { Stamped Secondary } \\
\text { Sheet }\end{array}$ & $\begin{array}{c}\text { FerroSteel } \\
\text { Stamped } \\
\text { SecondarySheet }\end{array}$ & Weight & 1.500230029 & 16.2514765 & 0.085157306 \\
\hline Ferro & Steel & $\begin{array}{c}\text { Stamped Secondary } \\
\text { Sheet }\end{array}$ & Chrome Finish & Surface Area & 2.1711783 & 43.4658086 & 0.15263142 \\
\hline Ferro & Steel & $\begin{array}{c}\text { Stamped Secondary } \\
\text { Sheet }\end{array}$ & Powder Coating & Surface Area & 4.589747 & 86.1691345 & 0.44941253 \\
\hline Ferro & Steel & $\begin{array}{c}\text { Stamped Unspecified } \\
\text { Sheet }\end{array}$ & None & Weight & 2.614044871 & 28.8449788 & 0.797583869 \\
\hline Ferro & Steel & $\begin{array}{l}\text { Stamped Unspecified } \\
\text { Sheet }\end{array}$ & Chrome Finish & Surface Area & 2.1711783 & 43.4658086 & 0.15263142 \\
\hline Ferro & Steel & $\begin{array}{c}\text { Stamped Unspecified } \\
\text { Sheet }\end{array}$ & Powder Coating & Surface Area & 4.589747 & 86.1691345 & 0.44941253 \\
\hline NonFerro & Aluminum & $\begin{array}{l}\text { Cast Primary } \\
\text { Aluminum }\end{array}$ & None & Weight & 12.73806314 & 206.9299699 & 0.270352713 \\
\hline NonFerro & Aluminum & $\begin{array}{l}\text { Cast Primary } \\
\text { Aluminum }\end{array}$ & Machining & Weight & 0.904047712 & 13.77555958 & 0.056505831 \\
\hline NonFerro & Aluminum & $\begin{array}{l}\text { Cast Primary } \\
\text { Aluminum }\end{array}$ & Anodizing & Surface Area & 4.1081486 & 80.4417333 & 0.36007964 \\
\hline NonFerro & Aluminum & $\begin{array}{l}\text { Cast Primary } \\
\text { Aluminum }\end{array}$ & Powder Coating & Surface Area & 3.7361933 & 67.39207009 & 0.33612864 \\
\hline NonFerro & Aluminum & $\begin{array}{l}\text { Cast Primary } \\
\text { Aluminum }\end{array}$ & Enameling & Surface Area & 8.6750434 & 182.720006 & 0.70488909 \\
\hline NonFerro & Aluminum & $\begin{array}{l}\text { Extruded Primary } \\
\text { Aluminum }\end{array}$ & None & Weight & 13.26611266 & 218.3858213 & 0.30333773 \\
\hline NonFerro & Aluminum & $\begin{array}{l}\text { Extruded Primary } \\
\text { Aluminum }\end{array}$ & Machining & Weight & 0.904047712 & 13.77555958 & 0.056505831 \\
\hline NonFerro & Aluminum & $\begin{array}{l}\text { Extruded Primary } \\
\text { Aluminum }\end{array}$ & Anodizing & Surface Area & 4.1081486 & 80.4417333 & 0.36007964 \\
\hline NonFerro & Aluminum & $\begin{array}{l}\text { Extruded Primary } \\
\text { Aluminum }\end{array}$ & Powder Coating & Surface Area & 3.7361933 & 67.39207009 & 0.33612864 \\
\hline NonFerro & Aluminum & $\begin{array}{l}\text { Extruded Primary } \\
\text { Aluminum }\end{array}$ & Enameling & Surface Area & 8.6750434 & 182.720006 & 0.70488909 \\
\hline NonFerro & Aluminum & $\begin{array}{l}\text { Billet Primary } \\
\text { Aluminum }\end{array}$ & None & Weight & 12.29078358 & 198.5148286 & 0.259572984 \\
\hline NonFerro & Aluminum & $\begin{array}{l}\text { Billet Primary } \\
\text { Aluminum }\end{array}$ & Machining & Weight & 0.904047712 & 13.77555958 & 0.056505831 \\
\hline NonFerro & Aluminum & $\begin{array}{l}\text { Billet Primary } \\
\text { Aluminum }\end{array}$ & Anodizing & Surface Area & 4.1081486 & 80.4417333 & 0.36007964 \\
\hline NonFerro & Aluminum & $\begin{array}{l}\text { Billet Primary } \\
\text { Aluminum }\end{array}$ & Powder Coating & Surface Area & 3.7361933 & 67.39207009 & 0.33612864 \\
\hline
\end{tabular}




\begin{tabular}{|c|c|c|c|c|c|c|c|}
\hline $\begin{array}{l}\text { Material } \\
\text { Class }\end{array}$ & Material & Primary Process & $\begin{array}{l}\text { Secondary } \\
\text { Process }\end{array}$ & $\begin{array}{c}\text { Process } \\
\text { Measurable }\end{array}$ & $\begin{array}{c}\text { GHG } \\
\left(\mathrm{kg} \mathrm{CO}_{2} \mathrm{Eq}\right)\end{array}$ & $\begin{array}{c}\text { Energy } \\
\text { (MJ) }\end{array}$ & $\begin{array}{l}\text { Mercury } \\
(\mathrm{mg})\end{array}$ \\
\hline NonFerro & Aluminum & $\begin{array}{l}\text { Billet Primary } \\
\text { Aluminum }\end{array}$ & Enameling & Surface Area & 8.6750434 & 182.720006 & 0.70488909 \\
\hline NonFerro & Aluminum & $\begin{array}{c}\text { Cast Secondary } \\
\text { Aluminum }\end{array}$ & None & Weight & 1.376646768 & 24.90149175 & 0.187863233 \\
\hline NonFerro & Aluminum & $\begin{array}{l}\text { Cast Secondary } \\
\text { Aluminum }\end{array}$ & Machining & Weight & 0.904047712 & 13.77555958 & 0.056505831 \\
\hline NonFerro & Aluminum & $\begin{array}{l}\text { Cast Secondary } \\
\text { Aluminum }\end{array}$ & Anodizing & Surface Area & 4.1081486 & 80.4417333 & 0.36007964 \\
\hline NonFerro & Aluminum & $\begin{array}{c}\text { Cast Secondary } \\
\text { Aluminum }\end{array}$ & Powder Coating & Surface Area & 3.7361933 & 67.39207009 & 0.33612864 \\
\hline NonFerro & Aluminum & $\begin{array}{l}\text { Cast Secondary } \\
\text { Aluminum }\end{array}$ & Enameling & Surface Area & 8.6750434 & 182.720006 & 0.70488909 \\
\hline NonFerro & Aluminum & $\begin{array}{l}\text { Extruded Secondary } \\
\text { Aluminum }\end{array}$ & None & Weight & 1.904696293 & 36.35734318 & 0.220848249 \\
\hline NonFerro & Aluminum & $\begin{array}{l}\text { Extruded Secondary } \\
\text { Aluminum }\end{array}$ & Machining & Weight & 0.904047712 & 13.77555958 & 0.056505831 \\
\hline NonFerro & Aluminum & $\begin{array}{l}\text { Extruded Secondary } \\
\text { Aluminum }\end{array}$ & Anodizing & Surface Area & 4.1081486 & 80.4417333 & 0.36007964 \\
\hline NonFerro & Aluminum & $\begin{array}{c}\text { Extruded Secondary } \\
\text { Aluminum }\end{array}$ & Powder Coating & Surface Area & 3.7361933 & 67.39207009 & 0.33612864 \\
\hline NonFerro & Aluminum & $\begin{array}{l}\text { Extruded Secondary } \\
\text { Aluminum }\end{array}$ & Enameling & Surface Area & 8.6750434 & 182.720006 & 0.70488909 \\
\hline NonFerro & Aluminum & $\begin{array}{l}\text { Billet Secondary } \\
\text { Aluminum }\end{array}$ & None & Weight & 0.929367214 & 16.4863505 & 0.177083503 \\
\hline NonFerro & Aluminum & $\begin{array}{l}\text { Billet Secondary } \\
\text { Aluminum }\end{array}$ & Machining & Weight & 0.904047712 & 13.77555958 & 0.056505831 \\
\hline NonFerro & Aluminum & $\begin{array}{l}\text { Billet Secondary } \\
\text { Aluminum }\end{array}$ & Anodizing & Surface Area & 4.1081486 & 80.4417333 & 0.36007964 \\
\hline NonFerro & Aluminum & $\begin{array}{l}\text { Billet Secondary } \\
\text { Aluminum }\end{array}$ & Powder Coating & Surface Area & 3.7361933 & 67.39207009 & 0.33612864 \\
\hline NonFerro & Aluminum & $\begin{array}{l}\text { Billet Secondary } \\
\text { Aluminum }\end{array}$ & Enameling & Surface Area & 8.6750434 & 182.720006 & 0.70488909 \\
\hline NonFerro & Aluminum & $\begin{array}{l}\text { Cast Production Mix } \\
\text { Aluminum }\end{array}$ & None & Weight & 9.000655754 & 144.9634819 & 0.302132928 \\
\hline NonFerro & Aluminum & $\begin{array}{l}\text { Cast Production Mix } \\
\text { Aluminum }\end{array}$ & Machining & Weight & 0.904047712 & 13.77555958 & 0.056505831 \\
\hline NonFerro & Aluminum & $\begin{array}{l}\text { Cast Production Mix } \\
\text { Aluminum }\end{array}$ & Anodizing & Surface Area & 4.1081486 & 80.4417333 & 0.36007964 \\
\hline NonFerro & Aluminum & $\begin{array}{l}\text { Cast Production Mix } \\
\text { Aluminum }\end{array}$ & Powder Coating & Surface Area & 3.7361933 & 67.39207009 & 0.33612864 \\
\hline NonFerro & Aluminum & $\begin{array}{l}\text { Cast Production Mix } \\
\text { Aluminum }\end{array}$ & Enameling & Surface Area & 8.6750434 & 182.720006 & 0.70488909 \\
\hline NonFerro & Aluminum & $\begin{array}{l}\text { Extruded Production } \\
\text { Mix Aluminum }\end{array}$ & None & Weight & 9.528705279 & 156.4193333 & 0.335117944 \\
\hline NonFerro & Aluminum & $\begin{array}{l}\text { Extruded Production } \\
\text { Mix Aluminum }\end{array}$ & Machining & Weight & 0.904047712 & 13.77555958 & 0.056505831 \\
\hline NonFerro & Aluminum & $\begin{array}{l}\text { Extruded Production } \\
\text { Mix Aluminum }\end{array}$ & Anodizing & Surface Area & 4.1081486 & 80.4417333 & 0.36007964 \\
\hline NonFerro & Aluminum & $\begin{array}{l}\text { Extruded Production } \\
\text { Mix Aluminum }\end{array}$ & Powder Coating & Surface Area & 3.7361933 & 67.39207009 & 0.33612864 \\
\hline NonFerro & Aluminum & $\begin{array}{l}\text { Extruded Production } \\
\text { Mix Aluminum }\end{array}$ & Enameling & Surface Area & 8.6750434 & 182.720006 & 0.70488909 \\
\hline NonFerro & Aluminum & $\begin{array}{l}\text { Billet Production Mix } \\
\text { Aluminum }\end{array}$ & None & Weight & 8.5533762 & 136.5483407 & 0.291353198 \\
\hline NonFerro & Aluminum & $\begin{array}{l}\text { Billet Production Mix } \\
\text { Aluminum }\end{array}$ & Machining & Weight & 0.904047712 & 13.77555958 & 0.056505831 \\
\hline NonFerro & Aluminum & $\begin{array}{l}\text { Billet Production Mix } \\
\text { Aluminum }\end{array}$ & Anodizing & Surface Area & 4.1081486 & 80.4417333 & 0.36007964 \\
\hline NonFerro & Aluminum & $\begin{array}{l}\text { Billet Production Mix } \\
\text { Aluminum }\end{array}$ & Powder Coating & Surface Area & 3.7361933 & 67.39207009 & 0.33612864 \\
\hline NonFerro & Aluminum & $\begin{array}{c}\text { Billet Production Mix } \\
\text { Aluminum }\end{array}$ & Enameling & Surface Area & 8.6750434 & 182.720006 & 0.70488909 \\
\hline NonFerro & Aluminum & Drawn Aluminum & None & Weight & 11.38724456 & 190.5023852 & 0.278540064 \\
\hline NonFerro & Aluminum & Drawn Aluminum & Machining & Weight & 0.904047712 & 13.77555958 & 0.056505831 \\
\hline NonFerro & Aluminum & Drawn Aluminum & Anodizing & Surface Area & 4.1081486 & 80.4417333 & 0.36007964 \\
\hline
\end{tabular}




\begin{tabular}{|c|c|c|c|c|c|c|c|}
\hline $\begin{array}{c}\text { Material } \\
\text { Class } \\
\end{array}$ & Material & Primary Process & $\begin{array}{c}\text { Secondary } \\
\text { Process }\end{array}$ & $\begin{array}{c}\text { Process } \\
\text { Measurable } \\
\end{array}$ & $\begin{array}{c}\mathrm{GHG} \\
\left(\mathrm{kg} \mathrm{CO} \mathrm{CO}_{2} \mathrm{Eq}\right)\end{array}$ & $\begin{array}{c}\text { Energy } \\
\text { (MJ) }\end{array}$ & $\begin{array}{c}\text { Mercury } \\
(\mathrm{mg})\end{array}$ \\
\hline NonFerro & Aluminum & Drawn Aluminum & Powder Coating & Surface Area & 3.7361933 & 67.39207009 & 0.33612864 \\
\hline NonFerro & Aluminum & Drawn Aluminum & Enameling & Surface Area & 8.6750434 & 182.720006 & 0.70488909 \\
\hline NonFerro & Brass & Cast Brass & None & Weight & 3.762849667 & 59.91918069 & 0.677826354 \\
\hline NonFerro & Brass & Cast Brass & Chrome Finish & Surface Area & 2.1711783 & 43.4658086 & 0.15263142 \\
\hline NonFerro & Brass & Cast Brass & Tin Plating & Surface Area & 3.3501117 & 58.01940624 & 0.28850761 \\
\hline NonFerro & Brass & Cast Brass & Enameling & Surface Area & 8.6750434 & 182.720006 & 0.70488909 \\
\hline NonFerro & Brass & Sheet Rolled Brass & None & Weight & 4.664183632 & 78.34379382 & 0.742007615 \\
\hline NonFerro & Brass & Sheet Rolled Brass & Chrome Finish & Surface Area & 4.1081486 & 80.4417333 & 0.36007964 \\
\hline NonFerro & Brass & Sheet Rolled Brass & Tin Plating & Surface Area & 8.6750434 & 182.720006 & 0.70488909 \\
\hline NonFerro & Brass & Sheet Rolled Brass & Enameling & Surface Area & 0.146636779 & 3.184760172 & 0.007150525 \\
\hline NonFerro & Copper & Copper Wire & None & Weight & 5.318855091 & 100.1669913 & 1.38938936 \\
\hline Plastics & ABS & Injection Molded ABS & None & Weight & 4.858731219 & 120.46228 & 0.120746251 \\
\hline Plastics & $\mathrm{ABS}$ & Extruded ABS & None & Weight & 4.01525608 & 102.4547768 & 0.091408998 \\
\hline Plastics & PVC & Injection Molded PVC & None & Weight & 3.066171271 & 91.00052007 & 0.144399742 \\
\hline Plastics & PVC & Blow Molded PVC & None & Weight & 2.744336675 & 88.5828133 & 0.14703004 \\
\hline Plastics & PVC & Extruded PVC & None & Weight & 2.222696131 & 72.99301696 & 0.11506249 \\
\hline Plastics & Acrylic & $\begin{array}{l}\text { Injection Molded } \\
\text { Acrylic } \\
\end{array}$ & None & Weight & 7.299667581 & 157.0742597 & 0.146972393 \\
\hline Plastics & Acrylic & Blow Molded Acrylic & None & Weight & 6.977832985 & 154.6565529 & 0.149602691 \\
\hline Plastics & Polycarbonate & $\begin{array}{c}\text { Injection Molded } \\
\text { Polycarbonate }\end{array}$ & None & Weight & 8.36124089 & 158.2831065 & 0.677413273 \\
\hline Plastics & Polycarbonate & $\begin{array}{c}\text { Blow Molded } \\
\text { Polycarbonate }\end{array}$ & None & Weight & 8.039406295 & 155.8653997 & 0.68004357 \\
\hline Plastics & Polycarbonate & $\begin{array}{c}\text { Extruded } \\
\text { Polycarbonate }\end{array}$ & None & Weight & 7.51776575 & 140.2756033 & 0.64807602 \\
\hline Plastics & Rubber & Molded Rubber & None & Weight & 3.013579066 & 97.65598074 & 0.08562702 \\
\hline Plastics & Polypropelene & $\begin{array}{l}\text { Injection Molded } \\
\text { Polypropelene }\end{array}$ & None & Weight & 3.058698084 & 104.5752414 & 0.087275663 \\
\hline Plastics & Polypropelene & $\begin{array}{c}\text { Blow Molded } \\
\text { Polypropelene }\end{array}$ & None & Weight & 2.753688034 & 102.5207032 & 0.090278013 \\
\hline Plastics & Polypropelene & $\begin{array}{c}\text { Extruded } \\
\text { Polypropelene }\end{array}$ & None & Weight & 2.23204749 & 86.93090685 & 0.058310462 \\
\hline $\begin{array}{l}\text { Glass and } \\
\text { Ceramics }\end{array}$ & Glass & Glass Tube & None & Weight & 3.284090168 & 56.59662635 & 0.131814563 \\
\hline Paper & Cardboard & Cardboard Sheet & None & Weight & 0.82567448 & 29.94073804 & 0.057744944 \\
\hline Paper & Kraft Paper & Kraft Paper Sheet & None & Weight & -0.94070575 & 60.19813738 & 0.072827306 \\
\hline Electronics & Circuit Components & Populated SM PCB & None & Weight & 0.153001714 & 2.931618675 & 0.022340826 \\
\hline Electronics & Circuit Components & $\begin{array}{l}\text { Unpopulated } \\
\text { SM PCB }\end{array}$ & None & Surface Area & 282.47496 & 5441.992731 & 38.878574 \\
\hline Electronics & Circuit Components & $\begin{array}{l}\text { Unpopulated } \\
\text { SM PCB }\end{array}$ & $\begin{array}{l}\text { Soldering - } \\
\text { Lead Free }\end{array}$ & Surface Area & 7.2936526 & 137.6922946 & 1.7052234 \\
\hline Electronics & Circuit Components & $\begin{array}{l}\text { Unpopulated } \\
\text { SM PCB }\end{array}$ & $\begin{array}{c}\text { Soldering - } \\
\text { Lead Containing }\end{array}$ & Surface Area & 6.0504967 & 113.9800679 & 1.5989887 \\
\hline Electronics & Circuit Components & $\begin{array}{c}\text { Populated Through } \\
\text { Mount PCB } \\
\end{array}$ & None & Weight & 0.153001714 & 2.931618675 & 0.022340826 \\
\hline Electronics & Circuit Components & SMD Resistor & None & Weight & 32.978617 & 636.082929 & 5.2443102 \\
\hline Electronics & Circuit Components & $\begin{array}{l}\text { Metal Film Through } \\
\text { Mount Resistor }\end{array}$ & None & Weight & 32.978617 & 636.082929 & 5.2443102 \\
\hline Electronics & Circuit Components & $\begin{array}{c}\text { Wirewound Through } \\
\text { Mount Resistor }\end{array}$ & None & Weight & 30.672732 & 591.907292 & 5.0370355 \\
\hline Electronics & Circuit Components & SMD Transistor & None & Weight & 147.2454 & 2945.170993 & 15.626699 \\
\hline Electronics & Circuit Components & $\begin{array}{c}\text { Through Mount } \\
\text { Transistor }\end{array}$ & None & Weight & 146.29456 & 2926.569262 & 15.61097 \\
\hline Electronics & Circuit Components & SMD Capacitor & None & Weight & 59.81028 & 1147.877364 & 8.9218276 \\
\hline Electronics & Circuit Components & $\begin{array}{l}\text { Through Mount } \\
\text { Capacitor }\end{array}$ & None & Weight & 51.102405 & 996.215663 & 6.3847818 \\
\hline Electronics & Circuit Components & Ring Core Inductor & None & Weight & 43.870914 & 835.485269 & 6.5259277 \\
\hline
\end{tabular}




\begin{tabular}{|c|c|c|c|c|c|c|c|}
\hline $\begin{array}{c}\text { Material } \\
\text { Class }\end{array}$ & Material & Primary Process & $\begin{array}{c}\text { Secondary } \\
\text { Process }\end{array}$ & $\begin{array}{c}\text { Process } \\
\text { Measurable }\end{array}$ & $\begin{array}{c}\text { GHG } \\
\left(\mathrm{kg} \mathrm{CO} \mathrm{CO}_{2} \mathrm{Eq}\right)\end{array}$ & $\begin{array}{c}\text { Energy } \\
\text { (MJ) }\end{array}$ & $\begin{array}{l}\text { Mercury } \\
(\mathrm{mg})\end{array}$ \\
\hline Electronics & Circuit Components & $\begin{array}{c}\text { SMD Integrated } \\
\text { Circuit Small }\end{array}$ & None & Weight & 1018.5318 & 16302.96763 & 359.16752 \\
\hline Electronics & Circuit Components & SMD Diode & None & Weight & 231.92488 & 4702.522543 & 19.329606 \\
\hline Electronics & Circuit Components & Through Mount Diode & None & Weight & 231.19182 & 4685.986548 & 18.541785 \\
\hline Electronics & Circuit Components & SMD LED & None & Weight & 231.92488 & 4702.522543 & 19.329606 \\
\hline Electronics & Circuit Components & Through Mount LED & None & Weight & 231.19182 & 4685.986548 & 18.541785 \\
\hline Electronics & $\begin{array}{l}\text { Other Electronic } \\
\text { Components }\end{array}$ & Silicone Potting & None & Weight & 2.6980774 & 62.63038164 & 0.24630457 \\
\hline Electronics & $\begin{array}{l}\text { Other Electronic } \\
\text { Components }\end{array}$ & Cooling Fan & None & Weight & 11.901097 & 254.3552561 & 2.1100731 \\
\hline Electronics & $\begin{array}{c}\text { Other Electronic } \\
\text { Components }\end{array}$ & $\begin{array}{c}\text { Fluorescent Lamp } \\
\text { Ballast } \\
\end{array}$ & None & Life & 1.869034873 & 32.91482576 & 0.408868228 \\
\hline Other & Adhesives & Epoxy & None & Weight & 7.585504804 & 139.0339859 & 1.114017364 \\
\hline Other & Adhesives & Thermal Adhesive & None & Weight & 2.6980774 & 62.63038164 & 0.24630457 \\
\hline Other & Fasteners & Steel Fastener & None & Weight & 3.110835263 & 46.90177909 & 1.051343551 \\
\hline Other & Fasteners & Brass Fastener & None & Weight & 4.87102084 & 81.71177313 & 1.331914429 \\
\hline Other & Fasteners & Plastic Fastener & None & Weight & 8.36124089 & 157.8805372 & 0.677413273 \\
\hline Other & Insulated Wire & PVC Insulated Wire & None & Weight & 3.894912589 & 91.40237032 & 1.417837416 \\
\hline Other & Insulated Wire & $\begin{array}{c}\text { Silicone Insulated } \\
\text { Wire }\end{array}$ & None & Weight & 3.053636983 & 64.55928151 & 0.943821178 \\
\hline Other & $\begin{array}{l}\text { Chemicals and } \\
\text { Minerals }\end{array}$ & Mercury & None & Weight & 117.92968 & 1758.135349 & 161001.04 \\
\hline Other & $\begin{array}{l}\text { Chemicals and } \\
\text { Minerals }\end{array}$ & Tungsten Wire & None & Weight & 22.81832859 & 248.0143005 & 0.115880345 \\
\hline Other & $\begin{array}{l}\text { Chemicals and } \\
\text { Minerals }\end{array}$ & Argon Gas & None & Weight & 0.227980001 & 4.596129455 & 0.014263145 \\
\hline $\begin{array}{l}\text { Assembled } \\
\text { Components }\end{array}$ & $\begin{array}{c}\text { Edison Screw Base } \\
\text { Assembly }\end{array}$ & Steel Edison Base & None & Weight & 1.407084522 & 20.06128553 & 0.309681255 \\
\hline $\begin{array}{l}\text { Assembled } \\
\text { Components }\end{array}$ & $\begin{array}{c}\text { Edison Screw Base } \\
\text { Assembly }\end{array}$ & Brass Edison Base & None & Weight & 2.340741192 & 40.37800833 & 0.520891069 \\
\hline
\end{tabular}

\begin{tabular}{|l|l|l|l|l|r|r|r|}
\hline Energy & Energy Use & US Grid Mix & None & Energy & 0.213479418 & 3.49897215 & 0.00399156 \\
\hline Energy & Energy Use & EU Grid Mix & None & Energy & 0.146636779 & 3.184760172 & 0.007150525 \\
\hline
\end{tabular}

\begin{tabular}{|c|c|c|c|c|r|r|r|}
\hline End of Life & Waste Disposal & Landfill & None & Weight & 0.7 & 0.314 & 1.44 \\
\hline End of Life & Waste Disposal & Incineration & None & Weight & 0.00529 & 0.0923 & 0.00101 \\
\hline End of Life & Reclamation & Recycled & None & Weight & $\begin{array}{l}\text { Data Not } \\
\text { Available }\end{array}$ & $\begin{array}{l}\text { Data Not } \\
\text { Available }\end{array}$ & $\begin{array}{l}\text { Data Not } \\
\text { Available }\end{array}$ \\
\hline End of Life & Reclamation & Remanufactured & None & Weight & $\begin{array}{l}\text { Data Not } \\
\text { Available }\end{array}$ & $\begin{array}{l}\text { Data Not } \\
\text { Available }\end{array}$ & $\begin{array}{l}\text { Data Not } \\
\text { Available }\end{array}$ \\
\hline
\end{tabular}




\section{Impact Documentation}

Below are the sources of the data used for the above environmental impact values.

\begin{tabular}{|c|c|c|c|c|c|c|c|c|c|c|c|}
\hline \multirow[b]{2}{*}{$\begin{array}{l}\text { Material } \\
\text { Class }\end{array}$} & \multirow[b]{2}{*}{ Material } & \multirow[b]{2}{*}{$\begin{array}{l}\text { Process } \\
\text { Name }\end{array}$} & \multirow[b]{2}{*}{$\begin{array}{l}\text { Description } \\
\text { of Data } \\
\text { Source }\end{array}$} & \multicolumn{4}{|c|}{ SimaPro } & \multicolumn{4}{|c|}{$\mathrm{GaBi}$} \\
\hline & & & & Title & $\begin{array}{l}\text { Method } \\
- \text { GHG }\end{array}$ & $\begin{array}{l}\text { Method - } \\
\text { Energy }\end{array}$ & $\begin{array}{l}\text { Method - } \\
\text { Mercury }\end{array}$ & Title & $\begin{array}{l}\text { Method - } \\
\text { GHG }\end{array}$ & $\begin{array}{l}\text { Method - } \\
\text { Energy }\end{array}$ & $\begin{array}{l}\text { Method - } \\
\text { Mercury }\end{array}$ \\
\hline Ferro & Steel & Steel Billet & $\begin{array}{l}\text { Average of } \\
\text { values from } \\
\text { SimPro and } \\
\text { GaBi }\end{array}$ & $\begin{array}{l}\text { Analyzing } \\
1 \text { kg 'Steel, } \\
\text { low-alloyed, } \\
\text { at plant/ } \\
\text { RER S' }\end{array}$ & $\begin{array}{l}\text { IPCC } \\
2007 \\
\text { GWP } \\
100 a \\
\text { V1.01 }\end{array}$ & $\begin{array}{l}\text { Cumulative } \\
\text { Energy } \\
\text { Demand } \\
\text { V1.05 I } \\
\text { Cumulative } \\
\text { energy } \\
\text { demand }\end{array}$ & Inventory ${ }^{*}$ & $\begin{array}{l}\text { DE: Steel } \\
\text { cold rolled } \\
\mathrm{PE}\end{array}$ & $\begin{array}{l}\text { CML2001, } \\
\text { Global } \\
\text { Warming } \\
\text { Potential } \\
\text { (GWP } 100 \\
\text { years) [kg } \\
\text { CO2- } \\
\text { Equiv.] kg } \\
\text { CO2-Equiv. }\end{array}$ & $\begin{array}{l}\text { Energy } \\
\text { renewable } \\
\text { and non- } \\
\text { renewable } \\
\text { (gross } \\
\text { calorific } \\
\text { value) [MJ] }\end{array}$ & Inventory* \\
\hline Ferro & Steel & $\begin{array}{l}\text { Wire } \\
\text { Drawing }\end{array}$ & $\begin{array}{l}\text { Values from } \\
\text { SimaPro }\end{array}$ & $\begin{array}{l}\text { Analyzing } \\
1 \mathrm{~kg} \text { 'Wire } \\
\text { drawing, } \\
\text { steel/RER S' }\end{array}$ & $\begin{array}{l}\text { IPCC } \\
2007 \\
\text { GWP } \\
100 a \\
\text { V1.01 }\end{array}$ & $\begin{array}{l}\text { Cumulative } \\
\text { Energy } \\
\text { Demand } \\
\text { V1.05 / } \\
\text { Cumulative } \\
\text { energy } \\
\text { demand } \\
\end{array}$ & Inventory* & & & & \\
\hline Ferro & Steel & Milling & $\begin{array}{l}\text { Average of } \\
\text { values from } \\
\text { SimPro and } \\
\text { GaBi }\end{array}$ & $\begin{array}{l}\text { Analyzing } \\
1 \mathrm{~kg} \text { 'Milling, } \\
\text { steel, } \\
\text { average/ } \\
\text { RER S' }\end{array}$ & $\begin{array}{l}\text { IPCC } \\
2007 \\
\text { GWP } \\
100 a \\
\text { V1.01 }\end{array}$ & $\begin{array}{l}\text { Cumulative } \\
\text { Energy } \\
\text { Demand } \\
\text { V1.05 I } \\
\text { Cumulative } \\
\text { energy } \\
\text { demand }\end{array}$ & Inventory ${ }^{*}$ & $\begin{array}{l}\text { GLO: Steel } \\
\text { high- } \\
\text { alloyed } \\
\text { machining } \\
(0,47 \mathrm{~kg} \\
\text { shavings } \\
\text { per } 1 \mathrm{~kg} \\
\text { part) PE [b] }\end{array}$ & $\begin{array}{l}\text { CML2001, } \\
\text { Global } \\
\text { Warming } \\
\text { Potential } \\
\text { (GWP 100 } \\
\text { years) [kg } \\
\text { CO2- } \\
\text { Equiv.] kg } \\
\text { CO2-Equiv. }\end{array}$ & $\begin{array}{l}\text { Energy } \\
\text { renewable } \\
\text { and non- } \\
\text { renewable } \\
\text { (gross } \\
\text { calorific } \\
\text { value) [MJ] }\end{array}$ & Inventory* \\
\hline Ferro & Steel & $\begin{array}{l}\text { Chrome } \\
\text { Finish }\end{array}$ & $\begin{array}{l}\text { Values from } \\
\text { SimaPro }\end{array}$ & $\begin{array}{l}\text { Analyzing } \\
1 \mathrm{~m} 2 \\
\text { 'Selective } \\
\text { coating, } \\
\text { copper sheet, } \\
\text { black } \\
\text { chrome/ } \\
\text { RER S' } \\
\end{array}$ & $\begin{array}{l}\text { IPCC } \\
2007 \\
\text { GWP } \\
100 a \\
\text { V1.02 }\end{array}$ & $\begin{array}{l}\text { Cumulative } \\
\text { Energy } \\
\text { Demand } \\
\text { V1.05 / } \\
\text { Cumulative } \\
\text { energy } \\
\text { demand }\end{array}$ & Inventory* & & & & \\
\hline Ferro & Steel & $\begin{array}{l}\text { Powder } \\
\text { Coating }\end{array}$ & $\begin{array}{l}\text { Values from } \\
\text { SimaPro }\end{array}$ & $\begin{array}{l}\text { Analyzing } \\
1 \mathrm{~m} 2 \\
\text { 'Powder } \\
\text { coating, } \\
\text { steel/RER S' }\end{array}$ & $\begin{array}{l}\text { IPCC } \\
2007 \\
\text { GWP } \\
100 a \\
\text { V1.03 }\end{array}$ & $\begin{array}{l}\text { Cumulative } \\
\text { Energy } \\
\text { Demand } \\
\text { V1.05 / } \\
\text { Cumulative } \\
\text { energy } \\
\text { demand } \\
\end{array}$ & Inventory ${ }^{*}$ & & & & \\
\hline Ferro & Steel & $\begin{array}{l}\text { Stamped } \\
\text { Primary } \\
\text { Sheet }\end{array}$ & $\begin{array}{l}\text { Average of } \\
\text { values from } \\
\text { SimPro and } \\
\text { GaBi }\end{array}$ & $\begin{array}{l}\text { Comparing } \\
1 \mathrm{~kg} \text { 'Steel, } \\
\text { low-alloyed, } \\
\text { at plant/RER } \\
\mathrm{S}^{\prime} \text { with } 1 \mathrm{~kg} \\
\text { 'Sheet rolling, } \\
\text { steel/RER S' }\end{array}$ & $\begin{array}{l}\text { IPCC } \\
2007 \\
\text { GWP } \\
100 a \\
\text { V1.04 }\end{array}$ & $\begin{array}{l}\text { Cumulative } \\
\text { Energy } \\
\text { Demand } \\
\text { V1.05 / } \\
\text { Cumulative } \\
\text { energy } \\
\text { demand }\end{array}$ & Inventory* & $\begin{array}{l}\text { DE: Steel } \\
\text { sheet } \\
\text { (ECCS) } \\
\text { BUWAL }\end{array}$ & $\begin{array}{l}\text { CML2001, } \\
\text { Global } \\
\text { Warming } \\
\text { Potential } \\
\text { (GWP } 100 \\
\text { years) [kg } \\
\text { CO2- } \\
\text { Equiv.] kg } \\
\text { CO2-Equiv. }\end{array}$ & $\begin{array}{l}\text { Energy } \\
\text { renewable } \\
\text { and non- } \\
\text { renewable } \\
\text { (gross } \\
\text { calorific } \\
\text { value) [MJ] }\end{array}$ & Inventory* \\
\hline Ferro & Steel & $\begin{array}{l}\text { FerroSteel } \\
\text { Stamped } \\
\text { Secondary } \\
\text { Sheet }\end{array}$ & $\begin{array}{l}\text { Values from } \\
\text { GaBi }\end{array}$ & & & & & $\begin{array}{l}\text { DE: Steel } \\
\text { sheet } \\
\text { secondary } \\
\text { (ECCS) } \\
\text { BUWAL }\end{array}$ & $\begin{array}{l}\text { CML2001, } \\
\text { Global } \\
\text { Warming } \\
\text { Potential } \\
\text { (GWP 100 } \\
\text { years) [kg } \\
\text { CO2- } \\
\text { Equiv.] kg } \\
\text { CO2-Equiv. }\end{array}$ & $\begin{array}{l}\text { Energy } \\
\text { renewable } \\
\text { and non- } \\
\text { renewable } \\
\text { (gross } \\
\text { calorific } \\
\text { value) [MJ] }\end{array}$ & Inventory* \\
\hline Ferro & Steel & $\begin{array}{l}\text { Stamped } \\
\text { Unspecifie } \\
\text { d Sheet }\end{array}$ & $\begin{array}{l}\text { Average of } \\
\text { average of } \\
\text { Primary and } \\
\text { Secondary } \\
\text { values from } \\
\text { SimPro and } \\
\text { GaBi }\end{array}$ & & & & & & & & \\
\hline
\end{tabular}




\begin{tabular}{|c|c|c|c|c|c|c|c|c|c|c|c|}
\hline & & & & \multirow{2}{*}{\multicolumn{4}{|c|}{ SimaPro }} & & & & \\
\hline & & & & & & & & \multicolumn{4}{|c|}{ GaBi } \\
\hline $\begin{array}{l}\text { Material } \\
\text { Class }\end{array}$ & Material & $\begin{array}{l}\text { Process } \\
\text { Name }\end{array}$ & $\begin{array}{l}\text { Description } \\
\text { of Data } \\
\text { Source }\end{array}$ & Title & $\begin{array}{l}\text { Method } \\
- \text { GHG }\end{array}$ & $\begin{array}{l}\text { Method - } \\
\text { Energy }\end{array}$ & $\begin{array}{l}\text { Method - } \\
\text { Mercury }\end{array}$ & Title & $\begin{array}{l}\text { Method - } \\
\text { GHG }\end{array}$ & $\begin{array}{l}\text { Method - } \\
\text { Energy }\end{array}$ & $\begin{array}{l}\text { Method - } \\
\text { Mercury }\end{array}$ \\
\hline NonFerro & Aluminum & $\begin{array}{l}\text { Cast } \\
\text { Primary } \\
\text { Aluminum }\end{array}$ & $\begin{array}{l}\text { Primary } \\
\text { Aluminum } \\
\text { Raw Material } \\
+ \text { Aluminum } \\
\text { Casting } \\
\end{array}$ & & & & & & & & \\
\hline NonFerro & Aluminum & $\begin{array}{l}\text { Primary } \\
\text { Aluminum } \\
\text { Raw } \\
\text { Material }\end{array}$ & $\begin{array}{l}\text { Average of } \\
\text { values from } \\
\text { SimPro and } \\
\mathrm{GaBi}\end{array}$ & $\begin{array}{l}\text { Analyzing } \\
1 \mathrm{~kg} \\
\text { 'Aluminum, } \\
\text { primary, at } \\
\text { plant/RER S' }\end{array}$ & $\begin{array}{l}\text { IPCC } \\
2007 \\
\text { GWP } \\
\text { 100a } \\
\text { V1.02 }\end{array}$ & $\begin{array}{l}\text { Cumulative } \\
\text { Energy } \\
\text { Demand } \\
\text { V1.05 / } \\
\text { Cumulative } \\
\text { energy } \\
\text { demand }\end{array}$ & Inventory* & $\begin{array}{l}\text { RER: } \\
\text { Aluminum } \\
\text { ingot mix } \\
\text { PE }\end{array}$ & $\begin{array}{l}\text { CML2001, } \\
\text { Global } \\
\text { Warming } \\
\text { Potential } \\
\text { (GWP 100 } \\
\text { years) [kg } \\
\text { CO2- } \\
\text { Equiv.] kg } \\
\text { CO2-Equiv. }\end{array}$ & $\begin{array}{l}\text { Energy } \\
\text { renewable } \\
\text { and non- } \\
\text { renewable } \\
\text { (gross } \\
\text { calorific } \\
\text { value) [MJ] }\end{array}$ & Inventory ${ }^{*}$ \\
\hline NonFerro & Aluminum & $\begin{array}{l}\text { Aluminum } \\
\text { Casting }\end{array}$ & $\begin{array}{l}\text { Average of } \\
\text { values from } \\
\text { SimPro and } \\
\mathrm{GaBi}\end{array}$ & $\begin{array}{l}\text { Analyzing } \\
1 \mathrm{~kg} \\
\text { 'Casting, } \\
\text { bronze/CH S' }\end{array}$ & $\begin{array}{l}\text { IPCC } \\
2007 \\
\text { GWP } \\
100 a \\
\text { V1.03 }\end{array}$ & $\begin{array}{l}\text { Cumulative } \\
\text { Energy } \\
\text { Demand } \\
\text { V1.05 / } \\
\text { Cumulative } \\
\text { energy } \\
\text { demand }\end{array}$ & Inventory* & $\begin{array}{l}\text { DE: } \\
\text { Aluminum } \\
\text { die-cast } \\
\text { part PE [b] }\end{array}$ & $\begin{array}{l}\text { In this methoo } \\
\text { energy are rer } \\
\text { and electrical } \\
\text { datasets ident } \\
\text { items. }\end{array}$ & $\begin{array}{l}\text {, only electrical } \\
\text { orted. Impacts } \\
\text { energy are adde } \\
\text { ified in the follov }\end{array}$ & $\begin{array}{l}\text { ind thermal } \\
\text { om thermal } \\
\text { d from those } \\
\text { ing two line }\end{array}$ \\
\hline NonFerro & Aluminum & $\begin{array}{l}\text { Electrical } \\
\text { energy }\end{array}$ & $\begin{array}{l}\text { Impact } \\
\text { values from } \\
\text { GaBi are } \\
\text { used to } \\
\text { populate } \\
\text { impacts for } \\
\text { Aluminum } \\
\text { Casting }\end{array}$ & & & & & $\begin{array}{l}\text { EU-25: } \\
\text { Power grid } \\
\text { mix PE }\end{array}$ & $\begin{array}{l}\text { CML2001, } \\
\text { Global } \\
\text { Warming } \\
\text { Potential } \\
\text { (GWP 100 } \\
\text { years) [kg } \\
\text { CO2- } \\
\text { Equiv.] kg } \\
\text { CO2-Equiv. } \\
\end{array}$ & $\begin{array}{l}\text { Energy } \\
\text { renewable } \\
\text { and non- } \\
\text { renewable } \\
\text { (gross } \\
\text { calorific } \\
\text { value) [MJ] }\end{array}$ & Inventory* \\
\hline NonFerro & Aluminum & $\begin{array}{l}\text { Thermal } \\
\text { energy - } \\
\text { unspecified }\end{array}$ & $\begin{array}{l}\text { Impact } \\
\text { values from } \\
\text { GaBi are } \\
\text { used to } \\
\text { populate } \\
\text { impacts for } \\
\text { Aluminum } \\
\text { Casting. } \\
\text { Because the } \\
\text { source is not } \\
\text { specified, all } \\
\text { thermal } \\
\text { energy } \\
\text { sources are } \\
\text { averaged. }\end{array}$ & & & & & $\begin{array}{l}\text { Thermal } \\
\text { energy } \\
\text { from } \\
\text { unspecified } \\
\text { fuel }\end{array}$ & $\begin{array}{l}\text { Values are av } \\
\text { thermal sourc }\end{array}$ & $\begin{array}{l}\text { eraged from the } \\
\text { s }\end{array}$ & elow \\
\hline NonFerro & Aluminum & $\begin{array}{l}\text { Thermal } \\
\text { energy } \\
\text { from coal }\end{array}$ & $\begin{array}{l}\text { Impact } \\
\text { values are } \\
\text { used to } \\
\text { populate } \\
\text { "Thermal } \\
\text { energy - } \\
\text { unspecified" }\end{array}$ & & & & & $\begin{array}{l}\text { Thermal } \\
\text { energy } \\
\text { from coal } \\
\text { BUWAL }\end{array}$ & $\begin{array}{l}\text { CML2001, } \\
\text { Global } \\
\text { Warming } \\
\text { Potential } \\
\text { (GWP 100 } \\
\text { years) [kg } \\
\text { CO2- } \\
\text { Equiv.] kg } \\
\text { CO2-Equiv. }\end{array}$ & $\begin{array}{l}\text { Energy } \\
\text { renewable } \\
\text { and non- } \\
\text { renewable } \\
\text { (gross } \\
\text { calorific } \\
\text { value) [MJ] }\end{array}$ & Inventory* \\
\hline NonFerro & Aluminum & $\begin{array}{l}\text { Thermal } \\
\text { energy } \\
\text { from diesel }\end{array}$ & $\begin{array}{l}\text { Impact } \\
\text { values are } \\
\text { used to } \\
\text { populate } \\
\text { "Thermal } \\
\text { energy - } \\
\text { unspecified" }\end{array}$ & & & & & $\begin{array}{l}\text { Thermal } \\
\text { energy } \\
\text { from diesel } \\
\text { BUWAL }\end{array}$ & $\begin{array}{l}\text { CML2001, } \\
\text { Global } \\
\text { Warming } \\
\text { Potential } \\
\text { (GWP 100 } \\
\text { years) [kg } \\
\text { CO2- } \\
\text { Equiv.] kg } \\
\text { CO2-Equiv. }\end{array}$ & $\begin{array}{l}\text { Energy } \\
\text { renewable } \\
\text { and non- } \\
\text { renewable } \\
\text { (gross } \\
\text { calorific } \\
\text { value) [MJ] }\end{array}$ & Inventory* \\
\hline NonFerro & Aluminum & $\begin{array}{l}\text { Thermal } \\
\text { energy } \\
\text { from gas }\end{array}$ & $\begin{array}{l}\text { Impact } \\
\text { values are } \\
\text { used to } \\
\text { populate } \\
\text { "Thermal } \\
\text { energy - } \\
\text { unspecified" }\end{array}$ & & & & & $\begin{array}{l}\text { Thermal } \\
\text { energy } \\
\text { from gas } \\
\text { BUWAL }\end{array}$ & $\begin{array}{l}\text { CML2001, } \\
\text { Global } \\
\text { Warming } \\
\text { Potential } \\
\text { (GWP 100 } \\
\text { years) [kg } \\
\text { CO2- } \\
\text { Equiv.] kg } \\
\text { CO2-Equiv. }\end{array}$ & $\begin{array}{l}\text { Energy } \\
\text { renewable } \\
\text { and non- } \\
\text { renewable } \\
\text { (gross } \\
\text { calorific } \\
\text { value) [MJ] }\end{array}$ & Inventory* \\
\hline
\end{tabular}




\begin{tabular}{|c|c|c|c|c|c|c|c|c|c|c|c|}
\hline \multirow[b]{2}{*}{$\begin{array}{l}\text { Material } \\
\text { Class }\end{array}$} & \multirow[b]{2}{*}{ Material } & \multirow[b]{2}{*}{$\begin{array}{l}\text { Process } \\
\text { Name }\end{array}$} & \multirow[b]{2}{*}{$\begin{array}{l}\text { Description } \\
\text { of Data } \\
\text { Source } \\
\end{array}$} & \multicolumn{4}{|c|}{ SimaPro } & \multicolumn{4}{|c|}{ GaBi } \\
\hline & & & & Title & $\begin{array}{l}\text { Method } \\
\text { - GHG }\end{array}$ & $\begin{array}{l}\text { Method - } \\
\text { Energy }\end{array}$ & $\begin{array}{l}\text { Method - } \\
\text { Mercury }\end{array}$ & Title & $\begin{array}{l}\text { Method - } \\
\text { GHG }\end{array}$ & $\begin{array}{l}\text { Method - } \\
\text { Energy }\end{array}$ & $\begin{array}{l}\text { Method - } \\
\text { Mercury }\end{array}$ \\
\hline NonFerro & Aluminum & $\begin{array}{l}\text { Thermal } \\
\text { energy } \\
\text { from heavy } \\
\text { fuel oil }\end{array}$ & $\begin{array}{l}\text { Impact } \\
\text { values are } \\
\text { used to } \\
\text { populate } \\
\text { "Thermal } \\
\text { energy - } \\
\text { unspecified" }\end{array}$ & & & & & $\begin{array}{l}\text { Thermal } \\
\text { energy from } \\
\text { heavy fuel } \\
\text { oil BUWAL }\end{array}$ & $\begin{array}{l}\text { CML2001, } \\
\text { Global } \\
\text { Warming } \\
\text { Potential } \\
\text { (GWP } 100 \\
\text { years) [kg } \\
\text { CO2- } \\
\text { Equiv.] kg } \\
\text { CO2- } \\
\text { Equiv. }\end{array}$ & $\begin{array}{l}\text { Energy } \\
\text { renewable } \\
\text { and non- } \\
\text { renewable } \\
\text { (gross } \\
\text { calorific } \\
\text { value) [MJ] }\end{array}$ & Inventory* \\
\hline NonFerro & Aluminum & $\begin{array}{l}\text { Thermal } \\
\text { energy } \\
\text { from light } \\
\text { fuel oil }\end{array}$ & $\begin{array}{l}\text { Impact } \\
\text { values are } \\
\text { used to } \\
\text { populate } \\
\text { "Thermal } \\
\text { energy - } \\
\text { unspecified" }\end{array}$ & & & & & $\begin{array}{l}\text { Thermal } \\
\text { energy from } \\
\text { light fuel oil } \\
\text { BUWAL }\end{array}$ & $\begin{array}{l}\text { CML2001, } \\
\text { Global } \\
\text { Warming } \\
\text { Potential } \\
\text { (GWP 100 } \\
\text { years) [kg } \\
\text { CO2- } \\
\text { Equiv.] kg } \\
\text { CO2- } \\
\text { Equiv. } \\
\end{array}$ & $\begin{array}{l}\text { Energy } \\
\text { renewable } \\
\text { and non- } \\
\text { renewable } \\
\text { (gross } \\
\text { calorific } \\
\text { value) [MJ] }\end{array}$ & Inventory* \\
\hline NonFerro & Aluminum & $\begin{array}{l}\text { Thermal } \\
\text { energy } \\
\text { from } \\
\text { natural gas }\end{array}$ & $\begin{array}{l}\text { Impact } \\
\text { values are } \\
\text { used to } \\
\text { populate } \\
\text { "Thermal } \\
\text { energy - } \\
\text { unspecified" }\end{array}$ & & & & & $\begin{array}{l}\text { Thermal } \\
\text { energy from } \\
\text { natural gas } \\
\text { BUWAL }\end{array}$ & $\begin{array}{l}\text { CML2001, } \\
\text { Global } \\
\text { Warming } \\
\text { Potential } \\
\text { (GWP 100 } \\
\text { years) [kg } \\
\text { CO2- } \\
\text { Equiv.] kg } \\
\text { CO2- } \\
\text { Equiv. }\end{array}$ & $\begin{array}{l}\text { Energy } \\
\text { renewable } \\
\text { and non- } \\
\text { renewable } \\
\text { (gross } \\
\text { calorific } \\
\text { value) [MJ] }\end{array}$ & Inventory* \\
\hline NonFerro & Aluminum & $\begin{array}{l}\text { Thermal } \\
\text { energy } \\
\text { from wood }\end{array}$ & $\begin{array}{l}\text { Impact } \\
\text { values are } \\
\text { used to } \\
\text { populate } \\
\text { "Thermal } \\
\text { energy - } \\
\text { unspecified" }\end{array}$ & & & & & $\begin{array}{l}\text { Thermal } \\
\text { energy from } \\
\text { wood } \\
\text { BUWAL }\end{array}$ & $\begin{array}{l}\text { CML2001, } \\
\text { Global } \\
\text { Warming } \\
\text { Potential } \\
\text { (GWP } 100 \\
\text { years) [kg } \\
\text { CO2- } \\
\text { Equiv.] kg } \\
\text { CO2- } \\
\text { Equiv. }\end{array}$ & $\begin{array}{l}\text { Energy } \\
\text { renewable } \\
\text { and non- } \\
\text { renewable } \\
\text { (gross } \\
\text { calorific } \\
\text { value) [MJ] }\end{array}$ & Inventory* \\
\hline NonFerro & Aluminum & Machining & $\begin{array}{l}\text { Average of } \\
\text { values from } \\
\text { SimPro and } \\
\mathrm{GaBi}\end{array}$ & $\begin{array}{l}\text { Analyzing } \\
1 \mathrm{~kg} \text { 'Milling, } \\
\text { aluminum, } \\
\text { average/ } \\
\text { RER S' }\end{array}$ & $\begin{array}{l}\text { IPCC } \\
2007 \\
\text { GWP } \\
100 a \\
\text { V1.03 }\end{array}$ & $\begin{array}{l}\text { Cumulative } \\
\text { Energy } \\
\text { Demand } \\
\text { V1.05 / } \\
\text { Cumulative } \\
\text { energy } \\
\text { demand }\end{array}$ & Inventory* & $\begin{array}{l}\text { DE: } \\
\text { Aluminum } \\
\text { cast part } \\
\text { machining } \\
\text { (standard) } \\
\text { PE [b] }\end{array}$ & $\begin{array}{l}\text { CML2001, } \\
\text { Global } \\
\text { Warming } \\
\text { Potential } \\
\text { (GWP 100 } \\
\text { years) [kg } \\
\text { CO2- } \\
\text { Equiv.] kg } \\
\text { CO2- } \\
\text { Equiv. }\end{array}$ & $\begin{array}{l}\text { Energy } \\
\text { renewable } \\
\text { and non- } \\
\text { renewable } \\
\text { (gross } \\
\text { calorific } \\
\text { value) [MJ] }\end{array}$ & Inventory* \\
\hline NonFerro & Aluminum & Anodizing & $\begin{array}{l}\text { Values from } \\
\text { SimaPro }\end{array}$ & $\begin{array}{l}\text { Analyzing } \\
1 \mathrm{~m} 2 \\
\text { 'Anodizing, } \\
\text { aluminum } \\
\text { sheet/RER S' }\end{array}$ & $\begin{array}{l}\text { IPCC } \\
2007 \\
\text { GWP } \\
100 a \\
\text { V1.04 }\end{array}$ & $\begin{array}{l}\text { Cumulative } \\
\text { Energy } \\
\text { Demand } \\
\text { V1.05 / } \\
\text { Cumulative } \\
\text { energy } \\
\text { demand } \\
\end{array}$ & Inventory* & & & & \\
\hline NonFerro & Aluminum & $\begin{array}{l}\text { Powder } \\
\text { Coating }\end{array}$ & $\begin{array}{l}\text { Values from } \\
\text { SimaPro }\end{array}$ & $\begin{array}{l}\text { Analyzing } \\
1 \mathrm{~m} 2 \\
\text { 'Powder } \\
\text { coating, } \\
\text { aluminum } \\
\text { sheet/RER S' }\end{array}$ & $\begin{array}{l}\text { IPCC } \\
2007 \\
\text { GWP } \\
100 a \\
\text { V1.05 }\end{array}$ & $\begin{array}{l}\text { Cumulative } \\
\text { Energy } \\
\text { Demand } \\
\text { V1.05 / } \\
\text { Cumulative } \\
\text { energy } \\
\text { demand } \\
\end{array}$ & Inventory ${ }^{*}$ & & & & \\
\hline NonFerro & Aluminum & Enameling & $\begin{array}{l}\text { Values from } \\
\text { SimaPro }\end{array}$ & $\begin{array}{l}\text { Analyzing } \\
1 \mathrm{~m} 2 \\
\text { 'Enameling/ } \\
\text { RER S' }\end{array}$ & $\begin{array}{l}\text { IPCC } \\
2007 \\
\text { GWP } \\
100 a \\
\text { V1.06 }\end{array}$ & $\begin{array}{l}\text { Cumulative } \\
\text { Energy } \\
\text { Demand } \\
\text { V1.05 / } \\
\text { Cumulative } \\
\text { energy } \\
\text { demand }\end{array}$ & Inventory ${ }^{*}$ & & & & \\
\hline
\end{tabular}




\begin{tabular}{|c|c|c|c|c|c|c|c|c|c|c|c|}
\hline \multirow[b]{2}{*}{$\begin{array}{l}\text { Material } \\
\text { Class }\end{array}$} & \multirow[b]{2}{*}{ Material } & \multirow[b]{2}{*}{$\begin{array}{l}\text { Process } \\
\text { Name }\end{array}$} & \multirow[b]{2}{*}{$\begin{array}{l}\text { Description } \\
\text { of Data } \\
\text { Source }\end{array}$} & \multicolumn{4}{|c|}{ SimaPro } & \multicolumn{4}{|c|}{$\mathrm{GaBi}$} \\
\hline & & & & Title & $\begin{array}{l}\text { Method } \\
\text { - GHG }\end{array}$ & $\begin{array}{l}\text { Method - } \\
\text { Energy }\end{array}$ & $\begin{array}{l}\text { Method - } \\
\text { Mercury }\end{array}$ & Title & $\begin{array}{l}\text { Method - } \\
\text { GHG }\end{array}$ & $\begin{array}{l}\text { Method - } \\
\text { Energy }\end{array}$ & $\begin{array}{l}\text { Method - } \\
\text { Mercury }\end{array}$ \\
\hline NonFerro & Aluminum & $\begin{array}{l}\text { Extruded } \\
\text { Primary } \\
\text { Aluminum }\end{array}$ & $\begin{array}{l}\text { Sum of } \\
\text { extruded } \\
\text { aluminum } \\
\text { process and } \\
\text { Primary } \\
\text { aluminum } \\
\text { billet process }\end{array}$ & $\begin{array}{l}\text { Analyzing } \\
1 \mathrm{~kg} \text { 'Section } \\
\text { bar } \\
\text { extrusion, } \\
\text { aluminum/ } \\
\text { RER S' }\end{array}$ & $\begin{array}{l}\text { IPCC } \\
2007 \\
\text { GWP } \\
100 a \\
\text { V1.07 }\end{array}$ & $\begin{array}{l}\text { Cumulative } \\
\text { Energy } \\
\text { Demand } \\
\text { V1.05 / } \\
\text { Cumulative } \\
\text { energy } \\
\text { demand }\end{array}$ & Inventory* & $\begin{array}{l}\text { RER: } \\
\text { Aluminum } \\
\text { extrusion } \\
\text { profile PE } \\
\text { [pl] }\end{array}$ & $\begin{array}{l}\text { CML2001, } \\
\text { Global } \\
\text { Warming } \\
\text { Potential } \\
\text { (GWP 100 } \\
\text { years) [kg } \\
\text { CO2- } \\
\text { Equiv.] kg } \\
\text { CO2-Equiv. }\end{array}$ & $\begin{array}{l}\text { Energy } \\
\text { renewable } \\
\text { and non- } \\
\text { renewable } \\
\text { (gross } \\
\text { calorific } \\
\text { value) [MJ] }\end{array}$ & Inventory* \\
\hline NonFerro & Aluminum & $\begin{array}{l}\text { Billet } \\
\text { Primary } \\
\text { Aluminum }\end{array}$ & $\begin{array}{l}\text { Average of } \\
\text { values from } \\
\text { SimPro and } \\
\text { GaBi }\end{array}$ & $\begin{array}{l}\text { Analyzing } \\
1 \mathrm{~kg} \\
\text { 'Aluminum, } \\
\text { primary, at } \\
\text { plant/RER S' }\end{array}$ & $\begin{array}{l}\text { IPCC } \\
2007 \\
\text { GWP } \\
100 a \\
\text { V1.08 }\end{array}$ & $\begin{array}{l}\text { Cumulative } \\
\text { Energy } \\
\text { Demand } \\
\text { V1.05 / } \\
\text { Cumulative } \\
\text { energy } \\
\text { demand }\end{array}$ & Inventory* & $\begin{array}{l}\text { RER: } \\
\text { Aluminum } \\
\text { ingot mix } \\
\text { PE }\end{array}$ & $\begin{array}{l}\text { CML2001, } \\
\text { Global } \\
\text { Warming } \\
\text { Potential } \\
\text { (GWP 100 } \\
\text { years) [kg } \\
\text { CO2- } \\
\text { Equiv.] kg } \\
\text { CO2-Equiv. }\end{array}$ & $\begin{array}{l}\text { Energy } \\
\text { renewable } \\
\text { and non-- } \\
\text { renewable } \\
\text { (gross } \\
\text { calorific } \\
\text { value) [MJ] }\end{array}$ & Inventory* \\
\hline NonFerro & Aluminum & $\begin{array}{l}\text { Cast } \\
\text { Secondary } \\
\text { Aluminum }\end{array}$ & $\begin{array}{l}\text { Sum of cast } \\
\text { aluminum } \\
\text { process and } \\
\text { secondary } \\
\text { aluminum } \\
\text { billet process } \\
\end{array}$ & & & & & & & & \\
\hline NonFerro & Aluminum & $\begin{array}{l}\text { Extruded } \\
\text { Secondary } \\
\text { Aluminum }\end{array}$ & $\begin{array}{l}\text { Sum of } \\
\text { extruded } \\
\text { aluminum } \\
\text { process and } \\
\text { secondary } \\
\text { aluminum } \\
\text { billet process }\end{array}$ & & & & & & & & \\
\hline NonFerro & Aluminum & $\begin{array}{l}\text { Billet } \\
\text { Secondary } \\
\text { Aluminum }\end{array}$ & $\begin{array}{l}\text { Average of } \\
\text { values from } \\
\text { SimPro and } \\
\mathrm{GaBi}\end{array}$ & $\begin{array}{l}\text { Analyzing } \\
1 \mathrm{~kg} \\
\text { 'Aluminum, } \\
\text { secondary, } \\
\text { from old } \\
\text { scrap, at } \\
\text { plant/ } \\
\text { RER S' }\end{array}$ & $\begin{array}{l}\text { IPCC } \\
2007 \\
\text { GWP } \\
100 a \\
\text { V1.08 }\end{array}$ & $\begin{array}{l}\text { Cumulative } \\
\text { Energy } \\
\text { Demand } \\
\text { V1.05 / } \\
\text { Cumulative } \\
\text { energy } \\
\text { demand }\end{array}$ & Inventory* & $\begin{array}{l}\text { RER: } \\
\text { Aluminum } \\
\text { ingot } \\
\text { secondary } \\
\text { BUWAL }\end{array}$ & $\begin{array}{l}\text { CML2001, } \\
\text { Global } \\
\text { Warming } \\
\text { Potential } \\
\text { (GWP 100 } \\
\text { years) [kg } \\
\text { CO2- } \\
\text { Equiv.] kg } \\
\text { CO2-Equiv. }\end{array}$ & \begin{tabular}{|l} 
Energy \\
renewable \\
and non- \\
renewable \\
(gross \\
calorific \\
value) [MJ]
\end{tabular} & Inventory ${ }^{*}$ \\
\hline NonFerro & Aluminum & $\begin{array}{l}\text { Cast } \\
\text { Production } \\
\text { Mix } \\
\text { Aluminum }\end{array}$ & $\begin{array}{l}\text { Sum of cast } \\
\text { aluminum } \\
\text { process and } \\
\text { production } \\
\text { mix aluminum } \\
\text { billet process }\end{array}$ & & & & & & & & \\
\hline NonFerro & Aluminum & $\begin{array}{l}\text { Extruded } \\
\text { Production } \\
\text { Mix } \\
\text { Aluminum }\end{array}$ & $\begin{array}{l}\text { Sum of } \\
\text { extruded } \\
\text { aluminum } \\
\text { process and } \\
\text { production } \\
\text { mix aluminum } \\
\text { billet process } \\
\end{array}$ & & & & & & & & \\
\hline NonFerro & Aluminum & $\begin{array}{l}\text { Billet } \\
\text { Production } \\
\text { Mix } \\
\text { Aluminum }\end{array}$ & $\begin{array}{l}\text { Values from } \\
\text { SimaPro }\end{array}$ & $\begin{array}{l}\text { Analyzing } \\
1 \mathrm{~kg} \\
\text { 'Aluminum, } \\
\text { production } \\
\text { mix, at } \\
\text { plant/RER S' }\end{array}$ & $\begin{array}{l}\text { IPCC } \\
2007 \\
\text { GWP } \\
100 a \\
\text { V1.08 }\end{array}$ & $\begin{array}{l}\text { Cumulative } \\
\text { Energy } \\
\text { Demand } \\
\text { V1.05 / } \\
\text { Cumulative } \\
\text { energy } \\
\text { demand } \\
\end{array}$ & Inventory* & & & & \\
\hline NonFerro & Aluminum & $\begin{array}{l}\text { Drawn } \\
\text { Aluminum }\end{array}$ & $\begin{array}{l}\text { Average of } \\
\text { values from } \\
\text { SimPro and } \\
\text { GaBi }\end{array}$ & $\begin{array}{l}\text { Analyzing } \\
1 \mathrm{~kg} \\
\text { 'Deformation } \\
\text { stroke, cold } \\
\text { impact } \\
\text { extrusion, } \\
\text { aluminum/ } \\
\text { RER S' }\end{array}$ & $\begin{array}{l}\text { IPCC } \\
2007 \\
\text { GWP } \\
100 a \\
\text { V1.09 }\end{array}$ & $\begin{array}{l}\text { Cumulative } \\
\text { Energy } \\
\text { Demand } \\
\text { V1.05 / } \\
\text { Cumulative } \\
\text { energy } \\
\text { demand }\end{array}$ & Inventory* & $\begin{array}{l}\text { DE: } \\
\text { Aluminum } \\
\text { sheet deep } \\
\text { drawing PE } \\
\text { [b] }\end{array}$ & $\begin{array}{l}\text { In this metho } \\
\text { reported. Imp } \\
\text { added from } \mathrm{t} \\
\text { Aluminum El }\end{array}$ & $\begin{array}{l}\text { only electrica } \\
\text { icts from elect } \\
\text { ose datasets ic } \\
\text { ctrical Energy I }\end{array}$ & $\begin{array}{l}\text { nergy is } \\
\text { cal energy is } \\
\text { ntified } \\
\text { e item above }\end{array}$ \\
\hline
\end{tabular}




\begin{tabular}{|c|c|c|c|c|c|c|c|c|c|c|c|}
\hline & \multirow{2}{*}{\multicolumn{4}{|c|}{ SimaPro }} & \multirow{2}{*}{\multicolumn{4}{|c|}{ GaBi }} \\
\hline & & & & & & & & & & & \\
\hline $\begin{array}{l}\text { Material } \\
\text { Class }\end{array}$ & Material & $\begin{array}{l}\text { Process } \\
\text { Name }\end{array}$ & $\begin{array}{l}\text { Description } \\
\text { of Data } \\
\text { Source }\end{array}$ & Title & $\begin{array}{l}\text { Method } \\
- \text { GHG }\end{array}$ & $\begin{array}{l}\text { Method - } \\
\text { Energy }\end{array}$ & $\begin{array}{l}\text { Method - } \\
\text { Mercury }\end{array}$ & Title & $\begin{array}{l}\text { Method - } \\
\text { GHG }\end{array}$ & $\begin{array}{l}\text { Method - } \\
\text { Energy }\end{array}$ & $\begin{array}{l}\text { Method - } \\
\text { Mercury }\end{array}$ \\
\hline NonFerro & Brass & Cast Brass & $\begin{array}{l}\text { Sum of cast } \\
\text { brass } \\
\text { process and } \\
\text { brass raw } \\
\text { material } \\
\text { process }\end{array}$ & & & & & & & & \\
\hline NonFerro & Brass & $\begin{array}{l}\text { Brass } \\
\text { casting } \\
\text { process }\end{array}$ & $\begin{array}{l}\text { Values from } \\
\text { SimaPro }\end{array}$ & $\begin{array}{l}\text { Analyzing } \\
1 \text { kg 'Casting, } \\
\text { brass } / \mathrm{CH} \mathrm{S}^{\prime}\end{array}$ & $\begin{array}{l}\text { IPCC } \\
2007 \\
\text { GWP } \\
100 a \\
\text { V1.09 }\end{array}$ & $\begin{array}{l}\text { Cumulative } \\
\text { Energy } \\
\text { Demand } \\
\text { V1.05 / } \\
\text { Cumulative } \\
\text { energy } \\
\text { demand }\end{array}$ & Inventory* & & & & \\
\hline NonFerro & Brass & $\begin{array}{l}\text { Brass raw } \\
\text { material }\end{array}$ & $\begin{array}{l}\text { Average of } \\
\text { values from } \\
\text { SimPro and } \\
\mathrm{GaBi}\end{array}$ & $\begin{array}{l}\text { Analyzing } \\
1 \mathrm{~kg} \text { 'Brass, } \\
\text { at plant } / \mathrm{CH} \\
\mathrm{S}^{\prime}\end{array}$ & $\begin{array}{l}\text { IPCC } \\
2007 \\
\text { GWP } \\
100 a \\
\text { V1.09 }\end{array}$ & $\begin{array}{l}\text { Cumulative } \\
\text { Energy } \\
\text { Demand } \\
\text { V1.05 / } \\
\text { Cumulative } \\
\text { energy } \\
\text { demand }\end{array}$ & Inventory* & $\begin{array}{l}\text { DE: Brass } \\
\text { PE }\end{array}$ & $\begin{array}{l}\text { CML2001, } \\
\text { Global } \\
\text { Warming } \\
\text { Potential } \\
\text { (GWP 100 } \\
\text { years) [kg } \\
\text { CO2- } \\
\text { Equiv.] kg } \\
\text { CO2- } \\
\text { Equiv. } \\
\end{array}$ & $\begin{array}{l}\text { Energy } \\
\text { renewable } \\
\text { and non- } \\
\text { renewable } \\
\text { (gross } \\
\text { calorific } \\
\text { value) [MJ] }\end{array}$ & Inventory* \\
\hline NonFerro & Brass & $\begin{array}{l}\text { Chrome } \\
\text { Finish }\end{array}$ & $\begin{array}{l}\text { Values from } \\
\text { SimaPro }\end{array}$ & $\begin{array}{l}\text { Analyzing } \\
1 \mathrm{~m} 2 \\
\text { 'Selective } \\
\text { coating, } \\
\text { copper } \\
\text { sheet, black } \\
\text { chrome/ } \\
\text { RER S' }\end{array}$ & $\begin{array}{l}\text { IPCC } \\
2007 \\
\text { GWP } \\
100 a \\
\text { V1.10 }\end{array}$ & $\begin{array}{l}\text { Cumulative } \\
\text { Energy } \\
\text { Demand } \\
\text { V1.05 / } \\
\text { Cumulative } \\
\text { energy } \\
\text { demand }\end{array}$ & Inventory* & & & & \\
\hline NonFerro & Brass & Tin Plating & $\begin{array}{l}\text { Values from } \\
\text { SimaPro }\end{array}$ & $\begin{array}{l}\text { Analyzing } \\
1 \mathrm{~m} 2 \text { 'Tin } \\
\text { plating, } \\
\text { pieces/ } \\
\text { RER S' }\end{array}$ & $\begin{array}{l}\text { IPCC } \\
2007 \\
\text { GWP } \\
100 a \\
\text { V1.11 }\end{array}$ & $\begin{array}{l}\text { Cumulative } \\
\text { Energy } \\
\text { Demand } \\
\text { V1.05 / } \\
\text { Cumulative } \\
\text { energy } \\
\text { demand }\end{array}$ & Inventory & & & & \\
\hline NonFerro & Brass & Enameling & $\begin{array}{l}\text { Values from } \\
\text { SimaPro }\end{array}$ & $\begin{array}{l}\text { Analyzing } \\
1 \mathrm{~m} 2 \\
\text { 'Enameling/ } \\
\text { RER S' }\end{array}$ & $\begin{array}{l}\text { IPCC } \\
2007 \\
\text { GWP } \\
100 a \\
\text { V1.06 }\end{array}$ & $\begin{array}{l}\text { Cumulative } \\
\text { Energy } \\
\text { Demand } \\
\text { V1.05 / } \\
\text { Cumulative } \\
\text { energy } \\
\text { demand }\end{array}$ & Inventory* & & & & \\
\hline NonFerro & Brass & $\begin{array}{l}\text { Sheet } \\
\text { Rolled } \\
\text { Brass }\end{array}$ & $\begin{array}{l}\text { Sum of sheet } \\
\text { rolled brass } \\
\text { process and } \\
\text { brass raw } \\
\text { material } \\
\text { process }\end{array}$ & & & & & & & & \\
\hline NonFerro & Brass & $\begin{array}{l}\text { Brass } \\
\text { sheet } \\
\text { rolling }\end{array}$ & $\begin{array}{l}\text { Average of } \\
\text { values from } \\
\text { SimPro and } \\
\mathrm{GaBi}\end{array}$ & $\begin{array}{l}\text { Analyzing } \\
1 \text { kg 'Sheet } \\
\text { rolling, } \\
\text { copperl } \\
\text { RER S' }\end{array}$ & $\begin{array}{l}\text { IPCC } \\
2007 \\
\text { GWP } \\
100 a \\
\text { V1.06 }\end{array}$ & $\begin{array}{l}\text { Cumulative } \\
\text { Energy } \\
\text { Demand } \\
\text { V1.05 / } \\
\text { Cumulative } \\
\text { energy } \\
\text { demand }\end{array}$ & Inventory & $\begin{array}{l}\text { RER: } \\
\text { Aluminum } \\
\text { sheet PE } \\
\text { [pl] }\end{array}$ & $\begin{array}{l}\text { CML2001, } \\
\text { Global } \\
\text { Warming } \\
\text { Potential } \\
\text { (GWP 100 } \\
\text { years) [kg } \\
\text { CO2- } \\
\text { Equiv.] kg } \\
\text { CO2- } \\
\text { Equiv. } \\
\end{array}$ & $\begin{array}{l}\text { Energy } \\
\text { renewable } \\
\text { and non- } \\
\text { renewable } \\
\text { (gross } \\
\text { calorific } \\
\text { value) [MJ] }\end{array}$ & Inventory* \\
\hline NonFerro & Copper & $\begin{array}{l}\text { Copper } \\
\text { Wire }\end{array}$ & $\begin{array}{l}\text { Sum of } \\
\text { copper wire } \\
\text { drawing } \\
\text { process and } \\
\text { copper raw } \\
\text { material } \\
\text { process }\end{array}$ & & & & & & & & \\
\hline
\end{tabular}




\begin{tabular}{|c|c|c|c|c|c|c|c|c|c|c|c|}
\hline \multirow[b]{2}{*}{$\begin{array}{l}\text { Material } \\
\text { Class }\end{array}$} & \multirow[b]{2}{*}{ Material } & \multirow[b]{2}{*}{$\begin{array}{l}\text { Process } \\
\text { Name }\end{array}$} & \multirow[b]{2}{*}{$\begin{array}{l}\text { Description } \\
\text { of Data } \\
\text { Source }\end{array}$} & \multicolumn{4}{|c|}{ SimaPro } & \multicolumn{4}{|c|}{ GaBi } \\
\hline & & & & Title & $\begin{array}{l}\text { Method } \\
- \text { GHG }\end{array}$ & $\begin{array}{l}\text { Method - } \\
\text { Energy }\end{array}$ & $\begin{array}{l}\text { Method - } \\
\text { Mercury }\end{array}$ & Title & $\begin{array}{l}\text { Method - } \\
\text { GHG }\end{array}$ & $\begin{array}{l}\text { Method - } \\
\text { Energy }\end{array}$ & $\begin{array}{l}\text { Method - } \\
\text { Mercury }\end{array}$ \\
\hline NonFerro & Copper & $\begin{array}{l}\text { Wire } \\
\text { drawing }\end{array}$ & $\begin{array}{l}\text { Average of } \\
\text { values from } \\
\text { SimPro and } \\
\text { GaBi }\end{array}$ & $\begin{array}{l}\text { Analyzing } \\
1 \mathrm{~kg} \text { 'Wire } \\
\text { drawing, } \\
\text { copperl } \\
\text { RER S' }\end{array}$ & $\begin{array}{l}\text { IPCC } \\
2007 \\
\text { GWP } \\
100 a \\
\text { V1.06 }\end{array}$ & $\begin{array}{l}\text { Cumulative } \\
\text { Energy } \\
\text { Demand } \\
\text { V1.05 / } \\
\text { Cumulative } \\
\text { energy } \\
\text { demand }\end{array}$ & Inventory* & $\begin{array}{l}\text { DE: } \\
\text { Copper } \\
\text { wire } \\
(0.06 \mathrm{~mm}) \\
\text { PE [b] }\end{array}$ & \multicolumn{3}{|c|}{$\begin{array}{l}\text { In this method, only electrical and thermal } \\
\text { energy are reported. Impacts from thermal } \\
\text { and electrical energy are added from those } \\
\text { datasets identified in the line items above } \\
\text { for aluminum casting }\end{array}$} \\
\hline NonFerro & Copper & $\begin{array}{l}\text { Raw } \\
\text { material }\end{array}$ & $\begin{array}{l}\text { Average of } \\
\text { values from } \\
\text { SimPro and } \\
\text { GaBi }\end{array}$ & $\begin{array}{l}\text { Analyzing } \\
1 \mathrm{~kg} \text { 'Copper, } \\
\text { primary, at } \\
\text { refineryl } \\
\text { GLO S' }\end{array}$ & $\begin{array}{l}\text { IPCC } \\
2007 \\
\text { GWP } \\
\text { 100a } \\
\text { V1.06 }\end{array}$ & $\begin{array}{l}\text { Cumulative } \\
\text { Energy } \\
\text { Demand } \\
\text { V1.05 I } \\
\text { Cumulative } \\
\text { energy } \\
\text { demand }\end{array}$ & Inventory* & $\begin{array}{l}\text { DE: } \\
\text { Copper mix } \\
(99,999 \% \\
\text { from } \\
\text { electrolysis } \\
\text { ) PE }\end{array}$ & $\begin{array}{l}\text { CML2001, } \\
\text { Global } \\
\text { Warming } \\
\text { Potential } \\
\text { (GWP 100 } \\
\text { years) [kg } \\
\text { CO2- } \\
\text { Equiv.] kg } \\
\text { CO2-Equiv. }\end{array}$ & $\begin{array}{l}\text { Energy } \\
\text { renewable } \\
\text { and non- } \\
\text { renewable } \\
\text { (gross } \\
\text { calorific } \\
\text { value) [MJ] }\end{array}$ & Inventory* \\
\hline Plastics & ABS & $\begin{array}{l}\text { Injection } \\
\text { Molded } \\
\text { ABS }\end{array}$ & $\begin{array}{l}\text { Sum of ABS } \\
\text { Raw Material } \\
\text { and Injection } \\
\text { Molding } \\
\text { processes }\end{array}$ & & & & & & & & \\
\hline Plastics & ABS & $\begin{array}{l}\text { Raw } \\
\text { Material }\end{array}$ & $\begin{array}{l}\text { Average of } \\
\text { values from } \\
\text { SimPro and } \\
\text { GaBi }\end{array}$ & $\begin{array}{l}\text { Analyzing } \\
1 \mathrm{~kg} \\
\text { 'Acrylonitrile- } \\
\text { butadiene- } \\
\text { styrene } \\
\text { copolymer, } \\
\text { ABS, at } \\
\text { plant/RER S' }\end{array}$ & $\begin{array}{l}\text { IPCC } \\
2007 \\
\text { GWP } \\
100 a \\
\text { V1.06 }\end{array}$ & $\begin{array}{l}\text { Cumulative } \\
\text { Energy } \\
\text { Demand } \\
\text { V1.05 I } \\
\text { Cumulative } \\
\text { energy } \\
\text { demand }\end{array}$ & Inventory* & $\begin{array}{l}\text { DE: } \\
\text { Acrylonitril } \\
\text { e- } \\
\text { butadiene- } \\
\text { styrene } \\
\text { granulate } \\
\text { mix (ABS) } \\
\text { PE }\end{array}$ & $\begin{array}{l}\text { CML2001, } \\
\text { Global } \\
\text { Warming } \\
\text { Potential } \\
\text { (GWP 100 } \\
\text { years) [kg } \\
\text { CO2- } \\
\text { Equiv.] kg } \\
\text { CO2-Equiv. }\end{array}$ & $\begin{array}{l}\text { Energy } \\
\text { renewable } \\
\text { and non- } \\
\text { renewable } \\
\text { (gross } \\
\text { calorific } \\
\text { value) [MJ] }\end{array}$ & Inventory* \\
\hline Plastics & & $\begin{array}{l}\text { Injection } \\
\text { Molding }\end{array}$ & $\begin{array}{l}\text { Average of } \\
\text { values from } \\
\text { SimPro and } \\
\mathrm{GaBi}\end{array}$ & $\begin{array}{l}\text { Analyzing } \\
1 \mathrm{~kg} \\
\text { 'Injection } \\
\text { molding/ } \\
\text { RER S' }\end{array}$ & $\begin{array}{l}\text { IPCC } \\
2007 \\
\text { GWP } \\
100 a \\
\text { V1.07 }\end{array}$ & $\begin{array}{l}\text { Cumulative } \\
\text { Energy } \\
\text { Demand } \\
\text { V1.05 / } \\
\text { Cumulative } \\
\text { energy } \\
\text { demand }\end{array}$ & Inventory* & $\begin{array}{l}\text { DE: Plastic } \\
\text { injection } \\
\text { molding } \\
\text { part } \\
\text { (unspecific) } \\
\text { PE [b] }\end{array}$ & $\begin{array}{l}\text { CML2001, } \\
\text { Global } \\
\text { Warming } \\
\text { Potential } \\
\text { (GWP 100 } \\
\text { years) [kg } \\
\text { CO2- } \\
\text { Equiv.] kg } \\
\text { CO2-Equiv. }\end{array}$ & $\begin{array}{l}\text { Energy } \\
\text { renewable } \\
\text { and non- } \\
\text { renewable } \\
\text { (gross } \\
\text { calorific } \\
\text { value) [MJ] }\end{array}$ & Inventory* \\
\hline Plastics & ABS & $\begin{array}{l}\text { Extruded } \\
\text { ABS }\end{array}$ & $\begin{array}{l}\text { Sum of ABS } \\
\text { Raw Material } \\
\text { and Extruding } \\
\text { processes }\end{array}$ & & & & & & & & \\
\hline Plastics & & Extruding & $\begin{array}{l}\text { Values from } \\
\text { SimaPro }\end{array}$ & $\begin{array}{l}\text { Analyzing } \\
1 \mathrm{~kg} \\
\text { 'Extrusion, } \\
\text { plastic } \\
\text { pipes/RER S' }\end{array}$ & $\begin{array}{l}\text { IPCC } \\
2007 \\
\text { GWP } \\
100 a \\
\text { V1.07 }\end{array}$ & $\begin{array}{l}\text { Cumulative } \\
\text { Energy } \\
\text { Demand } \\
\text { V1.05 / } \\
\text { Cumulative } \\
\text { energy } \\
\text { demand }\end{array}$ & Inventory* & & & & \\
\hline Plastics & PVC & $\begin{array}{l}\text { Injection } \\
\text { Molded } \\
\text { PVC }\end{array}$ & $\begin{array}{l}\text { Sum of PVC } \\
\text { Raw Material } \\
\text { and Injection } \\
\text { Molding } \\
\text { processes } \\
\end{array}$ & & & & & & & & \\
\hline Plastics & PVC & $\begin{array}{l}\text { Raw } \\
\text { Material }\end{array}$ & $\begin{array}{l}\text { Average of } \\
\text { values from } \\
\text { SimPro and } \\
\text { GaBi }\end{array}$ & $\begin{array}{l}\text { Analyzing } \\
1 \mathrm{~kg} \\
\text { 'Polyvinyl- } \\
\text { chloride, bulk } \\
\text { polymerized, } \\
\text { at plant/ } \\
\text { RER S' }\end{array}$ & $\begin{array}{l}\text { IPCC } \\
2007 \\
\text { GWP } \\
100 a \\
\text { V1.07 }\end{array}$ & $\begin{array}{l}\text { Cumulative } \\
\text { Energy } \\
\text { Demand } \\
\text { V1.05 / } \\
\text { Cumulative } \\
\text { energy } \\
\text { demand }\end{array}$ & Inventory* & $\begin{array}{l}\text { RER: } \\
\text { Polyvinylch } \\
\text { loride } \\
\text { powder } \\
\text { (PVC) } \\
\text { BUWAL }\end{array}$ & $\begin{array}{l}\text { CML2001, } \\
\text { Global } \\
\text { Warming } \\
\text { Potential } \\
\text { (GWP 100 } \\
\text { years) [kg } \\
\text { CO2- } \\
\text { Equiv.] kg } \\
\text { CO2-Equiv. }\end{array}$ & $\begin{array}{l}\text { Energy } \\
\text { renewable } \\
\text { and non- } \\
\text { renewable } \\
\text { (gross } \\
\text { calorific } \\
\text { value) [MJ] }\end{array}$ & Inventory* \\
\hline Plastics & PVC & $\begin{array}{l}\text { Blow } \\
\text { Molded } \\
\text { PVC }\end{array}$ & $\begin{array}{l}\text { Sum of PVC } \\
\text { Raw Material } \\
\text { and Blow } \\
\text { Molding } \\
\text { processes }\end{array}$ & & & & & & & & \\
\hline
\end{tabular}




\begin{tabular}{|c|c|c|c|c|c|c|c|c|c|c|c|}
\hline & \multirow{2}{*}{\multicolumn{4}{|c|}{ SimaPro }} & \multirow{2}{*}{\multicolumn{4}{|c|}{ GaBi }} \\
\hline & & & & & & & & & & & \\
\hline $\begin{array}{l}\text { Material } \\
\text { Class }\end{array}$ & Material & $\begin{array}{l}\text { Process } \\
\text { Name }\end{array}$ & $\begin{array}{l}\text { Description } \\
\text { of Data } \\
\text { Source }\end{array}$ & Title & $\begin{array}{l}\text { Method } \\
\text { - GHG }\end{array}$ & $\begin{array}{l}\text { Method - } \\
\text { Energy }\end{array}$ & $\begin{array}{l}\text { Method - } \\
\text { Mercury }\end{array}$ & Title & $\begin{array}{l}\text { Method - } \\
\text { GHG }\end{array}$ & $\begin{array}{l}\text { Method - } \\
\text { Energy }\end{array}$ & $\begin{array}{l}\text { Method - } \\
\text { Mercury }\end{array}$ \\
\hline Plastics & & $\begin{array}{l}\text { Blow } \\
\text { Molding }\end{array}$ & $\begin{array}{l}\text { Average of } \\
\text { values from } \\
\text { SimPro and } \\
\mathrm{GaBi}\end{array}$ & $\begin{array}{l}\text { Analyzing } \\
1 \mathrm{~kg} \text { 'Blow } \\
\text { molding/ } \\
\text { RER S' }\end{array}$ & $\begin{array}{l}\text { IPCC } \\
2007 \\
\text { GWP } \\
100 a \\
\text { V1.07 }\end{array}$ & $\begin{array}{l}\text { Cumulative } \\
\text { Energy } \\
\text { Demand } \\
\text { V1.05 / } \\
\text { Cumulative } \\
\text { energy } \\
\text { demand }\end{array}$ & Inventory* & $\begin{array}{l}\text { DE: Poly- } \\
\text { ethylene } \\
\text { (PE-HD) } \\
\text { blow } \\
\text { molding } \\
\text { PE [b] }\end{array}$ & $\begin{array}{l}\text { CML2001, } \\
\text { Global } \\
\text { Warming } \\
\text { Potential } \\
\text { (GWP 100 } \\
\text { years) [kg } \\
\text { CO2- } \\
\text { Equiv.] kg } \\
\text { CO2- } \\
\text { Equiv. } \\
\end{array}$ & $\begin{array}{l}\text { Energy } \\
\text { renewable } \\
\text { and non- } \\
\text { renewable } \\
\text { (gross } \\
\text { calorific } \\
\text { value) [MJ] }\end{array}$ & Inventory* \\
\hline Plastics & PVC & $\begin{array}{l}\text { Extruded } \\
\text { PVC }\end{array}$ & $\begin{array}{l}\text { Sum of PVC } \\
\text { Raw Material } \\
\text { and Extruding } \\
\text { processes }\end{array}$ & & & & & & & & \\
\hline Plastics & Acrylic & $\begin{array}{l}\text { Injection } \\
\text { Molded } \\
\text { Acrylic }\end{array}$ & $\begin{array}{l}\text { Sum of } \\
\text { Acrylic Raw } \\
\text { Material and } \\
\text { Injection } \\
\text { Molding } \\
\text { processes }\end{array}$ & & & & & & & & \\
\hline Plastics & Acrylic & $\begin{array}{l}\text { Raw } \\
\text { Material }\end{array}$ & $\begin{array}{l}\text { Average of } \\
\text { values from } \\
\text { SimPro and } \\
\mathrm{GaBi}\end{array}$ & $\begin{array}{l}\text { Analyzing } \\
1 \mathrm{~kg} \\
\text { 'Polymethyl } \\
\text { methacrylate, } \\
\text { beads, at } \\
\text { plant/RER S' }\end{array}$ & $\begin{array}{l}\text { IPCC } \\
2007 \\
\text { GWP } \\
100 a \\
\text { V1.07 }\end{array}$ & $\begin{array}{l}\text { Cumulative } \\
\text { Energy } \\
\text { Demand } \\
\text { V1.05/ } \\
\text { Cumulative } \\
\text { energy } \\
\text { demand }\end{array}$ & Inventory* & $\begin{array}{l}\text { DE: } \\
\text { Polymethyl } \\
\text { meth- } \\
\text { acrylate } \\
\text { granulate } \\
\text { (PMMA) PE }\end{array}$ & $\begin{array}{l}\text { CML2001, } \\
\text { Global } \\
\text { Warming } \\
\text { Potential } \\
\text { (GWP 100 } \\
\text { years) [kg } \\
\text { CO2- } \\
\text { Equiv.] kg } \\
\text { CO2- } \\
\text { Equiv. } \\
\end{array}$ & $\begin{array}{l}\text { Energy } \\
\text { renewable } \\
\text { and non- } \\
\text { renewable } \\
\text { (gross } \\
\text { calorific } \\
\text { value) [MJ] }\end{array}$ & Inventory* \\
\hline Plastics & Acrylic & $\begin{array}{l}\text { Blow } \\
\text { Molded } \\
\text { Acrylic }\end{array}$ & $\begin{array}{l}\text { Sum of } \\
\text { Acrylic Raw } \\
\text { Material and } \\
\text { Blow Molding } \\
\text { processes }\end{array}$ & & & & & & & & \\
\hline Plastics & $\begin{array}{l}\text { Poly- } \\
\text { carbonate }\end{array}$ & $\begin{array}{l}\text { Injection } \\
\text { Molded } \\
\text { Poly- } \\
\text { carbonate }\end{array}$ & $\begin{array}{l}\text { Sum of Poly- } \\
\text { carbonate } \\
\text { Raw Material } \\
\text { and Injection } \\
\text { Molding } \\
\text { processes } \\
\end{array}$ & & & & & & & & \\
\hline Plastics & $\begin{array}{l}\text { Poly- } \\
\text { carbonate }\end{array}$ & $\begin{array}{l}\text { Raw } \\
\text { Material }\end{array}$ & $\begin{array}{l}\text { Average of } \\
\text { values from } \\
\text { SimPro and } \\
\mathrm{GaBi}\end{array}$ & $\begin{array}{l}\text { Analyzing } \\
1 \mathrm{~kg} \text { 'Poly- } \\
\text { carbonate, at } \\
\text { plant/RER S' }\end{array}$ & $\begin{array}{l}\text { IPCC } \\
2007 \\
\text { GWP } \\
100 a \\
\text { V1.07 }\end{array}$ & $\begin{array}{l}\text { Cumulative } \\
\text { Energy } \\
\text { Demand } \\
\text { V1.05 / } \\
\text { Cumulative } \\
\text { energy } \\
\text { demand }\end{array}$ & Inventory* & $\begin{array}{l}\text { DE: } \\
\text { Polycarbon } \\
\text { ate } \\
\text { granulate } \\
\text { (PC) PE }\end{array}$ & $\begin{array}{l}\text { CML2001, } \\
\text { Global } \\
\text { Warming } \\
\text { Potential } \\
\text { (GWP 100 } \\
\text { years) [kg } \\
\text { CO2- } \\
\text { Equiv.] kg } \\
\text { CO2- } \\
\text { Equiv. }\end{array}$ & $\begin{array}{l}\text { Energy } \\
\text { renewable } \\
\text { and non- } \\
\text { renewable } \\
\text { (gross } \\
\text { calorific } \\
\text { value) [MJ] }\end{array}$ & Inventory* \\
\hline Plastics & $\begin{array}{l}\text { Poly- } \\
\text { carbonate }\end{array}$ & $\begin{array}{l}\text { Blow } \\
\text { Molded } \\
\text { Poly- } \\
\text { carbonate }\end{array}$ & $\begin{array}{l}\text { Sum of Poly- } \\
\text { carbonate } \\
\text { Raw Material } \\
\text { and Blow } \\
\text { Molding } \\
\text { processes } \\
\end{array}$ & & & & & & & & \\
\hline Plastics & $\begin{array}{l}\text { Poly- } \\
\text { carbonate }\end{array}$ & $\begin{array}{l}\text { Extruded } \\
\text { Poly- } \\
\text { carbonate }\end{array}$ & $\begin{array}{l}\text { Sum of Poly- } \\
\text { carbonate } \\
\text { Raw Material } \\
\text { and Extruding } \\
\text { processes }\end{array}$ & & & & & & & & \\
\hline Plastics & Rubber & $\begin{array}{l}\text { Molded } \\
\text { Rubber }\end{array}$ & $\begin{array}{l}\text { Average of } \\
\text { values from } \\
\text { SimPro and } \\
\mathrm{GaBi}\end{array}$ & $\begin{array}{l}\text { Analyzing } \\
1 \mathrm{~kg} \\
\text { 'Synthetic } \\
\text { rubber, at } \\
\text { plant/RER S' }\end{array}$ & $\begin{array}{l}\text { IPCC } \\
2007 \\
\text { GWP } \\
100 a \\
\text { V1.07 }\end{array}$ & $\begin{array}{l}\text { Cumulative } \\
\text { Energy } \\
\text { Demand } \\
\text { V1.05 I } \\
\text { Cumulative } \\
\text { energy } \\
\text { demand }\end{array}$ & Inventory* & $\begin{array}{l}\text { DE: } \\
\text { Styrene- } \\
\text { butadiene } \\
\text { rubber mix } \\
\text { (SBR) PE }\end{array}$ & $\begin{array}{l}\text { CML2001, } \\
\text { Global } \\
\text { Warming } \\
\text { Potential } \\
\text { (GWP 100 } \\
\text { years) [kg } \\
\text { CO2- } \\
\text { Equiv.] kg } \\
\text { CO2- } \\
\text { Equiv. }\end{array}$ & $\begin{array}{l}\text { Energy } \\
\text { renewable } \\
\text { and non- } \\
\text { renewable } \\
\text { (gross } \\
\text { calorific } \\
\text { value) [MJ] }\end{array}$ & Inventory* \\
\hline
\end{tabular}




\begin{tabular}{|c|c|c|c|c|c|c|c|c|c|c|c|}
\hline \multirow[b]{2}{*}{$\begin{array}{l}\text { Material } \\
\text { Class }\end{array}$} & \multirow[b]{2}{*}{ Material } & \multirow[b]{2}{*}{$\begin{array}{l}\text { Process } \\
\text { Name }\end{array}$} & \multirow[b]{2}{*}{$\begin{array}{l}\text { Description } \\
\text { of Data } \\
\text { Source }\end{array}$} & \multicolumn{4}{|c|}{ SimaPro } & \multicolumn{4}{|c|}{$\mathrm{GaBi}$} \\
\hline & & & & Title & $\begin{array}{l}\text { Method } \\
\text { - GHG }\end{array}$ & $\begin{array}{l}\text { Method - } \\
\text { Energy }\end{array}$ & $\begin{array}{l}\text { Method - } \\
\text { Mercury }\end{array}$ & Title & $\begin{array}{l}\text { Method - } \\
\text { GHG }\end{array}$ & $\begin{array}{l}\text { Method - } \\
\text { Energy }\end{array}$ & $\begin{array}{l}\text { Method - } \\
\text { Mercury }\end{array}$ \\
\hline Plastics & $\begin{array}{l}\text { Poly- } \\
\text { propelene }\end{array}$ & $\begin{array}{l}\text { Injection } \\
\text { Molded } \\
\text { Poly- } \\
\text { propelene }\end{array}$ & $\begin{array}{l}\text { Sum of Poly- } \\
\text { propelene } \\
\text { Raw Material } \\
\text { and Injection } \\
\text { Molding } \\
\text { processes } \\
\end{array}$ & & & & & & & & \\
\hline Plastics & $\begin{array}{l}\text { Poly- } \\
\text { propelene }\end{array}$ & $\begin{array}{l}\text { Raw } \\
\text { Material }\end{array}$ & $\begin{array}{l}\text { Average of } \\
\text { values from } \\
\text { SimPro and } \\
\text { GaBi }\end{array}$ & $\begin{array}{l}\text { Analyzing } \\
1 \mathrm{~kg} \text { 'Poly- } \\
\text { propylene, } \\
\text { granulate, at } \\
\text { plant/RER S' }\end{array}$ & $\begin{array}{l}\text { IPCC } \\
2007 \\
\text { GWP } \\
100 a \\
\text { V1.08 }\end{array}$ & $\begin{array}{l}\text { Cumulative } \\
\text { Energy } \\
\text { Demand } \\
\text { V1.05 / } \\
\text { Cumulative } \\
\text { energy } \\
\text { demand }\end{array}$ & Inventory* & $\begin{array}{l}\text { RER: Poly- } \\
\text { propylene } \\
\text { granulate } \\
\text { (PP) } \\
\text { BUWAL }\end{array}$ & $\begin{array}{l}\text { CML2001, } \\
\text { Global } \\
\text { Warming } \\
\text { Potential } \\
\text { (GWP 100 } \\
\text { years) [kg } \\
\text { CO2- } \\
\text { Equiv.] kg } \\
\text { CO2- } \\
\text { Equiv. }\end{array}$ & $\begin{array}{l}\text { Energy } \\
\text { renewable } \\
\text { and non- } \\
\text { renewable } \\
\text { (gross } \\
\text { calorific } \\
\text { value) [MJ] }\end{array}$ & Inventory* \\
\hline Plastics & $\begin{array}{l}\text { Poly- } \\
\text { propelene }\end{array}$ & $\begin{array}{l}\text { Blow } \\
\text { Molded } \\
\text { Poly- } \\
\text { propelene }\end{array}$ & $\begin{array}{l}\text { Sum of Poly- } \\
\text { propelene } \\
\text { Raw Material } \\
\text { and Blow } \\
\text { Molding } \\
\text { processes } \\
\end{array}$ & & & & & & & & \\
\hline Plastics & $\begin{array}{l}\text { Polypro- } \\
\text { pelene }\end{array}$ & $\begin{array}{l}\text { Extruded } \\
\text { Poly- } \\
\text { propelene }\end{array}$ & $\begin{array}{l}\text { Sum of Poly- } \\
\text { propelene } \\
\text { Raw Material } \\
\text { and Extruding } \\
\text { processes }\end{array}$ & & & & & & & & \\
\hline $\begin{array}{l}\text { Glass and } \\
\text { Ceramics }\end{array}$ & Glass & Glass Tube & $\begin{array}{l}\text { Values from } \\
\text { SimaPro }\end{array}$ & $\begin{array}{l}\text { Analyzing } \\
1 \mathrm{~kg} \text { 'Glass } \\
\text { tube, } \\
\text { borosilicate, } \\
\text { at plant/DE } \\
\mathrm{S}^{\prime}\end{array}$ & $\begin{array}{l}\text { IPCC } \\
2007 \\
\text { GWP } \\
100 a \\
\text { V1.08 }\end{array}$ & $\begin{array}{l}\text { Cumulative } \\
\text { Energy } \\
\text { Demand } \\
\text { V1.05 / } \\
\text { Cumulative } \\
\text { energy } \\
\text { demand }\end{array}$ & Inventory* & & & & \\
\hline Paper & Cardboard & $\begin{array}{l}\text { Cardboard } \\
\text { Sheet }\end{array}$ & $\begin{array}{l}\text { Values from } \\
\text { SimaPro }\end{array}$ & $\begin{array}{l}\text { Analyzing } \\
1 \mathrm{~kg} \\
\text { 'Packaging, } \\
\text { corrugated } \\
\text { board, mixed } \\
\text { fiber, single } \\
\text { wall, at } \\
\text { plant/RER S' }\end{array}$ & $\begin{array}{l}\text { IPCC } \\
2007 \\
\text { GWP } \\
100 a \\
\text { V1.09 }\end{array}$ & $\begin{array}{l}\text { Cumulative } \\
\text { Energy } \\
\text { Demand } \\
\text { V1.05 / } \\
\text { Cumulative } \\
\text { energy } \\
\text { demand }\end{array}$ & Inventory* & & & & \\
\hline Paper & Kraft Paper & $\begin{array}{l}\text { Kraft Paper } \\
\text { Sheet }\end{array}$ & $\begin{array}{l}\text { Values from } \\
\text { SimaPro }\end{array}$ & $\begin{array}{l}\text { Analyzing } \\
1 \mathrm{~kg} \text { 'Kraft } \\
\text { paper, } \\
\text { unbleached, } \\
\text { at plant/ } \\
\text { RER S' }\end{array}$ & $\begin{array}{l}\text { IPCC } \\
2007 \\
\text { GWP } \\
100 a \\
\text { V1.10 }\end{array}$ & $\begin{array}{l}\text { Cumulative } \\
\text { Energy } \\
\text { Demand } \\
\text { V1.05 / } \\
\text { Cumulative } \\
\text { energy } \\
\text { demand } \\
\end{array}$ & Inventory ${ }^{*}$ & & & & \\
\hline Electronics & $\begin{array}{l}\text { Circuit } \\
\text { Components }\end{array}$ & $\begin{array}{l}\text { Populated } \\
\text { SM PCB }\end{array}$ & $\begin{array}{l}\text { See } \\
\text { additional } \\
\text { information } \\
\text { below ** }\end{array}$ & & & & & & & & \\
\hline Electronics & $\begin{array}{l}\text { Circuit } \\
\text { Components }\end{array}$ & $\begin{array}{l}\text { Unpopu- } \\
\text { lated SM } \\
\text { PCB }\end{array}$ & $\begin{array}{l}\text { Values from } \\
\text { SimaPro }\end{array}$ & $\begin{array}{l}\text { Analyzing } \\
1 \mathrm{~m} 2 \text { 'Printed } \\
\text { wiring board, } \\
\text { surface } \\
\text { mount, at } \\
\text { plant/GLO S' }\end{array}$ & $\begin{array}{l}\text { IPCC } \\
2007 \\
\text { GWP } \\
100 a \\
\text { V1.10 }\end{array}$ & $\begin{array}{l}\text { Cumulative } \\
\text { Energy } \\
\text { Demand } \\
\text { V1.05 / } \\
\text { Cumulative } \\
\text { energy } \\
\text { demand }\end{array}$ & Inventory* & & & & \\
\hline Electronics & $\begin{array}{l}\text { Circuit } \\
\text { Components }\end{array}$ & $\begin{array}{l}\text { Soldering - } \\
\text { Lead Free }\end{array}$ & $\begin{array}{l}\text { Values from } \\
\text { SimaPro }\end{array}$ & $\begin{array}{l}\text { Analyzing } \\
1 \text { m2 } \\
\text { 'Mounting, } \\
\text { surface } \\
\text { mount } \\
\text { technology, } \\
\text { Pb-free } \\
\text { solder/ } \\
\text { GLO S' }\end{array}$ & $\begin{array}{l}\text { IPCC } \\
2007 \\
\text { GWP } \\
100 a \\
\text { V1.10 }\end{array}$ & $\begin{array}{l}\text { Cumulative } \\
\text { Energy } \\
\text { Demand } \\
\text { V1.05 / } \\
\text { Cumulative } \\
\text { energy } \\
\text { demand }\end{array}$ & Inventory* & & & & \\
\hline
\end{tabular}




\begin{tabular}{|c|c|c|c|c|c|c|c|c|c|c|c|}
\hline \multirow[b]{2}{*}{$\begin{array}{l}\text { Material } \\
\text { Class }\end{array}$} & \multirow[b]{2}{*}{ Material } & \multirow[b]{2}{*}{$\begin{array}{l}\text { Process } \\
\text { Name }\end{array}$} & \multirow[b]{2}{*}{$\begin{array}{l}\text { Description } \\
\text { of Data } \\
\text { Source }\end{array}$} & \multicolumn{4}{|c|}{ SimaPro } & \multicolumn{4}{|c|}{$\mathrm{GaBi}$} \\
\hline & & & & Title & $\begin{array}{l}\text { Method } \\
- \text { GHG }\end{array}$ & $\begin{array}{l}\text { Method - } \\
\text { Energy }\end{array}$ & $\begin{array}{l}\text { Method - } \\
\text { Mercury }\end{array}$ & Title & $\begin{array}{l}\text { Method - } \\
\text { GHG }\end{array}$ & $\begin{array}{l}\text { Method - } \\
\text { Energy }\end{array}$ & $\begin{array}{l}\text { Method - } \\
\text { Mercury }\end{array}$ \\
\hline Electronics & $\begin{array}{l}\text { Circuit } \\
\text { Components }\end{array}$ & $\begin{array}{l}\text { Soldering - } \\
\text { Lead } \\
\text { Containing }\end{array}$ & $\begin{array}{l}\text { Values from } \\
\text { SimaPro }\end{array}$ & $\begin{array}{l}\text { Analyzing } \\
1 \text { m2 } \\
\text { 'Mounting, } \\
\text { surface } \\
\text { mount } \\
\text { technology, } \\
\text { Pb- } \\
\text { containing } \\
\text { solder/ } \\
\text { GLO S' } \\
\end{array}$ & $\begin{array}{l}\text { IPCC } \\
2007 \\
\text { GWP } \\
100 a \\
\text { V1.11 }\end{array}$ & $\begin{array}{l}\text { Cumulative } \\
\text { Energy } \\
\text { Demand } \\
\text { V1.05 / } \\
\text { Cumulative } \\
\text { energy } \\
\text { demand }\end{array}$ & Inventory ${ }^{*}$ & & & & \\
\hline Electronics & $\begin{array}{l}\text { Circuit } \\
\text { Components }\end{array}$ & $\begin{array}{l}\text { Populated } \\
\text { Through } \\
\text { Mount PCB }\end{array}$ & $\begin{array}{l}\text { See } \\
\text { additional } \\
\text { information } \\
\text { below ** }\end{array}$ & & & & & & & & \\
\hline Electronics & $\begin{array}{l}\text { Circuit } \\
\text { Components }\end{array}$ & $\begin{array}{l}\text { SMD } \\
\text { Resistor }\end{array}$ & $\begin{array}{l}\text { Values from } \\
\text { SimaPro }\end{array}$ & $\begin{array}{l}\text { Analyzing } \\
1 \mathrm{~kg} \\
\text { 'Resistor, } \\
\text { metal film } \\
\text { type, } \\
\text { through-hole } \\
\text { mounting, at } \\
\text { plant/GLO S' }\end{array}$ & $\begin{array}{l}\text { IPCC } \\
2007 \\
\text { GWP } \\
100 a \\
\text { V1.11 }\end{array}$ & $\begin{array}{l}\text { Cumulative } \\
\text { Energy } \\
\text { Demand } \\
\text { V1.05 / } \\
\text { Cumulative } \\
\text { energy } \\
\text { demand }\end{array}$ & Inventory* & & & & \\
\hline Electronics & $\begin{array}{l}\text { Circuit } \\
\text { Components }\end{array}$ & $\begin{array}{l}\text { Metal Film } \\
\text { Through } \\
\text { Mount } \\
\text { Resistor }\end{array}$ & $\begin{array}{l}\text { Values from } \\
\text { SimaPro }\end{array}$ & $\begin{array}{l}\text { Analyzing } \\
1 \mathrm{~kg} \\
\text { 'Resistor, } \\
\text { metal film } \\
\text { type, } \\
\text { through-hole } \\
\text { mounting, at } \\
\text { plant/GLO S' }\end{array}$ & $\begin{array}{l}\text { IPCC } \\
2007 \\
\text { GWP } \\
100 a \\
\text { V1.11 }\end{array}$ & $\begin{array}{l}\text { Cumulative } \\
\text { Energy } \\
\text { Demand } \\
\text { V1.05/ } \\
\text { Cumulative } \\
\text { energy } \\
\text { demand }\end{array}$ & Inventory* & & & & \\
\hline Electronics & $\begin{array}{l}\text { Circuit } \\
\text { Components }\end{array}$ & $\begin{array}{l}\text { Wirewound } \\
\text { Through } \\
\text { Mount } \\
\text { Resistor }\end{array}$ & $\begin{array}{l}\text { Values from } \\
\text { SimaPro }\end{array}$ & $\begin{array}{l}\text { Analyzing } \\
1 \mathrm{~kg} \\
\text { 'Resistor, } \\
\text { wirewound, } \\
\text { through-hole } \\
\text { mounting, at } \\
\text { plant/GLO S' }\end{array}$ & $\begin{array}{l}\text { IPCC } \\
2007 \\
\text { GWP } \\
100 a \\
\text { V1.11 }\end{array}$ & $\begin{array}{l}\text { Cumulative } \\
\text { Energy } \\
\text { Demand } \\
\text { V1.05 / } \\
\text { Cumulative } \\
\text { energy } \\
\text { demand }\end{array}$ & Inventory* & & & & \\
\hline Electronics & $\begin{array}{l}\text { Circuit } \\
\text { Components }\end{array}$ & $\begin{array}{l}\text { SMD } \\
\text { Transistor }\end{array}$ & $\begin{array}{l}\text { Values from } \\
\text { SimaPro }\end{array}$ & $\begin{array}{l}\text { Analyzing } \\
1 \mathrm{~kg} \\
\text { 'Transistor, } \\
\text { SMD type, } \\
\text { surface } \\
\text { mounting, at } \\
\text { plant/GLO S' }\end{array}$ & $\begin{array}{l}\text { IPCC } \\
2007 \\
\text { GWP } \\
\text { 100a } \\
\text { V1.12 }\end{array}$ & $\begin{array}{l}\text { Cumulative } \\
\text { Energy } \\
\text { Demand } \\
\text { V1.05 / } \\
\text { Cumulative } \\
\text { energy } \\
\text { demand }\end{array}$ & Inventory* & & & & \\
\hline Electronics & $\begin{array}{l}\text { Circuit } \\
\text { Components }\end{array}$ & $\begin{array}{l}\text { Through } \\
\text { Mount } \\
\text { Transistor }\end{array}$ & $\begin{array}{l}\text { Values from } \\
\text { SimaPro }\end{array}$ & $\begin{array}{l}\text { Analyzing } \\
1 \mathrm{~kg} \\
\text { 'Transistor, } \\
\text { wired, small } \\
\text { size, } \\
\text { through-hole } \\
\text { mounting, at } \\
\text { plant/GLO S' }\end{array}$ & $\begin{array}{l}\text { IPCC } \\
2007 \\
\text { GWP } \\
\text { 100a } \\
\text { V1.13 }\end{array}$ & $\begin{array}{l}\text { Cumulative } \\
\text { Energy } \\
\text { Demand } \\
\text { V1.05 / } \\
\text { Cumulative } \\
\text { energy } \\
\text { demand }\end{array}$ & Inventory* & & & & \\
\hline Electronics & $\begin{array}{l}\text { Circuit } \\
\text { Components }\end{array}$ & $\begin{array}{l}\text { SMD } \\
\text { Capacitor }\end{array}$ & $\begin{array}{l}\text { Values from } \\
\text { SimaPro }\end{array}$ & $\begin{array}{l}\text { Analyzing } \\
1 \mathrm{~kg} \\
\text { 'Capacitor, } \\
\text { SMD type, } \\
\text { surface- } \\
\text { mounting, at } \\
\text { plant/GLO S' }\end{array}$ & $\begin{array}{l}\text { IPCC } \\
2007 \\
\text { GWP } \\
\text { 100a } \\
\text { V1.14 }\end{array}$ & $\begin{array}{l}\text { Cumulative } \\
\text { Energy } \\
\text { Demand } \\
\text { V1.05 / } \\
\text { Cumulative } \\
\text { energy } \\
\text { demand }\end{array}$ & Inventory* & & & & \\
\hline Electronics & $\begin{array}{l}\text { Circuit } \\
\text { Components }\end{array}$ & $\begin{array}{l}\text { Through } \\
\text { Mount } \\
\text { Capacitor }\end{array}$ & $\begin{array}{l}\text { Values from } \\
\text { SimaPro }\end{array}$ & $\begin{array}{l}\text { Analyzing } \\
1 \mathrm{~kg} \\
\text { 'Capacitor, } \\
\text { electrolyte } \\
\text { type, }<2 \mathrm{~cm} \\
\text { height, at } \\
\text { plant/GLO S' }\end{array}$ & $\begin{array}{l}\text { IPCC } \\
2007 \\
\text { GWP } \\
\text { 100a } \\
\text { V1.15 }\end{array}$ & $\begin{array}{l}\text { Cumulative } \\
\text { Energy } \\
\text { Demand } \\
\text { V1.05/ } \\
\text { Cumulative } \\
\text { energy } \\
\text { demand }\end{array}$ & Inventory* & & & & \\
\hline
\end{tabular}




\begin{tabular}{|c|c|c|c|c|c|c|c|c|c|c|c|}
\hline \multirow[b]{2}{*}{$\begin{array}{l}\text { Material } \\
\text { Class }\end{array}$} & \multirow[b]{2}{*}{ Material } & \multirow[b]{2}{*}{$\begin{array}{l}\text { Process } \\
\text { Name }\end{array}$} & \multirow[b]{2}{*}{$\begin{array}{l}\text { Description } \\
\text { of Data } \\
\text { Source }\end{array}$} & \multicolumn{4}{|c|}{ SimaPro } & \multicolumn{4}{|c|}{$\mathrm{GaBi}$} \\
\hline & & & & Title & $\begin{array}{l}\text { Method } \\
\text { - GHG }\end{array}$ & $\begin{array}{l}\text { Method - } \\
\text { Energy }\end{array}$ & $\begin{array}{l}\text { Method - } \\
\text { Mercury }\end{array}$ & Title & $\begin{array}{l}\text { Method - } \\
\text { GHG }\end{array}$ & $\begin{array}{l}\text { Method - } \\
\text { Energy }\end{array}$ & $\begin{array}{l}\text { Method- } \\
\text { Mercury }\end{array}$ \\
\hline Electronics & $\begin{array}{l}\text { Circuit } \\
\text { Components }\end{array}$ & $\begin{array}{l}\text { Ring Core } \\
\text { Inductor }\end{array}$ & $\begin{array}{l}\text { Values from } \\
\text { SimaPro }\end{array}$ & $\begin{array}{l}\text { Analyzing } \\
1 \mathrm{~kg} \\
\text { 'Inductor, } \\
\text { ring core } \\
\text { choke type, } \\
\text { at plant/ } \\
\text { GLO S' }\end{array}$ & $\begin{array}{l}\text { IPCC } \\
2007 \\
\text { GWP } \\
100 a \\
\text { V1.16 }\end{array}$ & $\begin{array}{l}\text { Cumulative } \\
\text { Energy } \\
\text { Demand } \\
\text { V1.05 / } \\
\text { Cumulative } \\
\text { energy } \\
\text { demand }\end{array}$ & Inventory* & & & & \\
\hline Electronics & $\begin{array}{l}\text { Circuit } \\
\text { Components }\end{array}$ & $\begin{array}{l}\text { SMD } \\
\text { Integrated } \\
\text { Circuit } \\
\text { Small }\end{array}$ & $\begin{array}{l}\text { Values from } \\
\text { SimaPro }\end{array}$ & $\begin{array}{l}\text { Analyzing } \\
1 \mathrm{~kg} \\
\text { 'Integrated } \\
\text { circuit, IC, } \\
\text { logic type, at } \\
\text { plant/GLO S' }\end{array}$ & $\begin{array}{l}\text { IPCC } \\
2007 \\
\text { GWP } \\
100 a \\
\text { V1.17 }\end{array}$ & $\begin{array}{l}\text { Cumulative } \\
\text { Energy } \\
\text { Demand } \\
\text { V1.05 / } \\
\text { Cumulative } \\
\text { energy } \\
\text { demand }\end{array}$ & Inventory* & & & & \\
\hline Electronics & $\begin{array}{l}\text { Circuit } \\
\text { Components }\end{array}$ & SMD Diode & $\begin{array}{l}\text { Values from } \\
\text { SimaPro }\end{array}$ & $\begin{array}{l}\text { Analyzing } \\
1 \mathrm{~kg} \text { 'Diode, } \\
\text { glass-, SMD } \\
\text { type, surface } \\
\text { mounting, at } \\
\text { plant/GLO S' }\end{array}$ & $\begin{array}{l}\text { IPCC } \\
2007 \\
\text { GWP } \\
100 a \\
\text { V1.18 }\end{array}$ & $\begin{array}{l}\text { Cumulative } \\
\text { Energy } \\
\text { Demand } \\
\text { V1.05 / } \\
\text { Cumulative } \\
\text { energy } \\
\text { demand }\end{array}$ & Inventory* & & & & \\
\hline Electronics & $\begin{array}{l}\text { Circuit } \\
\text { Components }\end{array}$ & $\begin{array}{l}\text { Through } \\
\text { Mount } \\
\text { Diode }\end{array}$ & $\begin{array}{l}\text { Values from } \\
\text { SimaPro }\end{array}$ & $\begin{array}{l}\text { Analyzing } \\
1 \mathrm{~kg} \text { 'Diode, } \\
\text { glass-, } \\
\text { through-hole } \\
\text { mounting, at } \\
\text { plant/GLO S' }\end{array}$ & $\begin{array}{l}\text { IPCC } \\
2007 \\
\text { GWP } \\
100 a \\
\text { V1.19 }\end{array}$ & $\begin{array}{l}\text { Cumulative } \\
\text { Energy } \\
\text { Demand } \\
\text { V1.05 I } \\
\text { Cumulative } \\
\text { energy } \\
\text { demand }\end{array}$ & Inventory* & & & & \\
\hline Electronics & $\begin{array}{l}\text { Circuit } \\
\text { Components }\end{array}$ & SMD LED & $\begin{array}{l}\text { Values from } \\
\text { SimaPro }\end{array}$ & $\begin{array}{l}\text { Analyzing } \\
1 \text { kg 'Light } \\
\text { emitting } \\
\text { diode, LED, } \\
\text { at plant/ } \\
\text { GLO S' }\end{array}$ & $\begin{array}{l}\text { IPCC } \\
2007 \\
\text { GWP } \\
100 a \\
\text { V1.20 }\end{array}$ & $\begin{array}{l}\text { Cumulative } \\
\text { Energy } \\
\text { Demand } \\
\text { V1.05 / } \\
\text { Cumulative } \\
\text { energy } \\
\text { demand }\end{array}$ & Inventory* & & & & \\
\hline Electronics & $\begin{array}{l}\text { Circuit } \\
\text { Components }\end{array}$ & $\begin{array}{l}\text { Through } \\
\text { Mount LED }\end{array}$ & $\begin{array}{l}\text { Values from } \\
\text { SimaPro }\end{array}$ & $\begin{array}{l}\text { Analyzing } \\
1 \text { kg 'Light } \\
\text { emitting } \\
\text { diode, LED, } \\
\text { at plant/ } \\
\text { GLO S' }\end{array}$ & $\begin{array}{l}\text { IPCC } \\
2007 \\
\text { GWP } \\
100 a \\
\text { V1.21 }\end{array}$ & $\begin{array}{l}\text { Cumulative } \\
\text { Energy } \\
\text { Demand } \\
\text { V1.05 / } \\
\text { Cumulative } \\
\text { energy } \\
\text { demand } \\
\end{array}$ & Inventory* & & & & \\
\hline Electronics & $\begin{array}{l}\text { Other } \\
\text { Electronic } \\
\text { Components }\end{array}$ & $\begin{array}{l}\text { Silicone } \\
\text { Potting }\end{array}$ & $\begin{array}{l}\text { Values from } \\
\text { SimaPro }\end{array}$ & $\begin{array}{l}\text { Analyzing } \\
1 \mathrm{~kg} \text { 'Silicone } \\
\text { product, at } \\
\text { plant/RER S' }\end{array}$ & $\begin{array}{l}\text { IPCC } \\
2007 \\
\text { GWP } \\
100 a \\
\text { V1.22 }\end{array}$ & $\begin{array}{l}\text { Cumulative } \\
\text { Energy } \\
\text { Demand } \\
\text { V1.05 / } \\
\text { Cumulative } \\
\text { energy } \\
\text { demand } \\
\end{array}$ & Inventory* & & & & \\
\hline Electronics & $\begin{array}{l}\text { Other } \\
\text { Electronic } \\
\text { Components }\end{array}$ & $\begin{array}{l}\text { Cooling } \\
\text { Fan }\end{array}$ & $\begin{array}{l}\text { Values from } \\
\text { SimaPro }\end{array}$ & $\begin{array}{l}\text { Analyzing } \\
1 \mathrm{~kg} \text { 'Fan, at } \\
\text { plant/GLO S' }\end{array}$ & $\begin{array}{l}\text { IPCC } \\
2007 \\
\text { GWP } \\
\text { 100a } \\
\text { V1.23 }\end{array}$ & $\begin{array}{l}\text { Cumulative } \\
\text { Energy } \\
\text { Demand } \\
\text { V1.05 I } \\
\text { Cumulative } \\
\text { energy } \\
\text { demand }\end{array}$ & Inventory* & & & & \\
\hline Electronics & $\begin{array}{l}\text { Other } \\
\text { Electronic } \\
\text { Components }\end{array}$ & $\begin{array}{l}\text { Fluorescen } \\
\text { t Lamp } \\
\text { Ballast }\end{array}$ & $\begin{array}{l}\text { See } \\
\text { additional } \\
\text { information } \\
\text { below }^{* *} \\
\end{array}$ & & & & & & & & \\
\hline Other & Adhesives & Epoxy & $\begin{array}{l}\text { Average of } \\
\text { values from } \\
\text { SimPro and } \\
\mathrm{GaBi}\end{array}$ & $\begin{array}{l}\text { Analyzing } \\
1 \text { kg 'Epoxy } \\
\text { resin, liquid, } \\
\text { at plant/ } \\
\text { RER S' }\end{array}$ & $\begin{array}{l}\text { IPCC } \\
2007 \\
\text { GWP } \\
100 a \\
\text { V1.23 }\end{array}$ & $\begin{array}{l}\text { Cumulative } \\
\text { Energy } \\
\text { Demand } \\
\text { V1.05 I } \\
\text { Cumulative } \\
\text { energy } \\
\text { demand }\end{array}$ & Inventory* & $\begin{array}{l}\text { RER: } \\
\text { Epoxy } \\
\text { resin } \\
\text { Plastics } \\
\text { Europe }\end{array}$ & $\begin{array}{l}\text { CML2001, } \\
\text { Global } \\
\text { Warming } \\
\text { Potential } \\
\text { (GWP 100 } \\
\text { years) [kg } \\
\text { CO2- } \\
\text { Equiv.] kg } \\
\text { CO2- } \\
\text { Equiv. }\end{array}$ & $\begin{array}{l}\text { Energy } \\
\text { renewable } \\
\text { and non- } \\
\text { renewable } \\
\text { (gross } \\
\text { calorific } \\
\text { value) [MJ] }\end{array}$ & Inventory* \\
\hline
\end{tabular}




\begin{tabular}{|c|c|c|c|c|c|c|c|c|c|c|c|}
\hline & \multirow{2}{*}{\multicolumn{4}{|c|}{ SimaPro }} & \multirow{2}{*}{\multicolumn{4}{|c|}{ GaBi }} \\
\hline & & & & & & & & & & & \\
\hline $\begin{array}{l}\text { Material } \\
\text { Class }\end{array}$ & Material & $\begin{array}{l}\text { Process } \\
\text { Name }\end{array}$ & $\begin{array}{l}\text { Description } \\
\text { of Data } \\
\text { Source }\end{array}$ & Title & $\begin{array}{l}\text { Method } \\
- \text { GHG }\end{array}$ & $\begin{array}{l}\text { Method - } \\
\text { Energy }\end{array}$ & $\begin{array}{l}\text { Method - } \\
\text { Mercury }\end{array}$ & Title & $\begin{array}{l}\text { Method - } \\
\text { GHG }\end{array}$ & $\begin{array}{l}\text { Method - } \\
\text { Energy }\end{array}$ & $\begin{array}{l}\text { Method - } \\
\text { Mercury }\end{array}$ \\
\hline Other & Adhesives & $\begin{array}{l}\text { Thermal } \\
\text { Adhesive }\end{array}$ & $\begin{array}{l}\text { Values from } \\
\text { SimaPro }\end{array}$ & $\begin{array}{l}\text { Analyzing } \\
1 \mathrm{~kg} \text { 'Silicone } \\
\text { product, at } \\
\text { plant/RER S' }\end{array}$ & $\begin{array}{l}\text { IPCC } \\
2007 \\
\text { GWP } \\
100 a \\
\text { V1.23 }\end{array}$ & $\begin{array}{l}\text { Cumulative } \\
\text { Energy } \\
\text { Demand } \\
\text { V1.05 / } \\
\text { Cumulative } \\
\text { energy } \\
\text { demand }\end{array}$ & Inventory* & & & & \\
\hline Other & Fasteners & $\begin{array}{l}\text { Steel } \\
\text { Fastener }\end{array}$ & $\begin{array}{l}\text { Sum of steel } \\
\text { billet and } \\
\text { machining } \\
\text { processes }\end{array}$ & & & & & & & & \\
\hline Other & Fasteners & $\begin{array}{l}\text { Brass } \\
\text { Fastener }\end{array}$ & $\begin{array}{l}\text { Sum of brass } \\
\text { billet and } \\
\text { machining } \\
\text { processes }\end{array}$ & & & & & & & & \\
\hline Other & Fasteners & $\begin{array}{l}\text { Plastic } \\
\text { Fastener }\end{array}$ & $\begin{array}{l}\text { Sum of poly- } \\
\text { carbonate } \\
\text { raw material } \\
\text { and injection } \\
\text { molding } \\
\text { processes }\end{array}$ & & & & & & & & \\
\hline Other & $\begin{array}{l}\text { Insulated } \\
\text { Wire }\end{array}$ & $\begin{array}{l}\text { PVC } \\
\text { Insulated } \\
\text { Wire }\end{array}$ & $\begin{array}{l}\text { Sum of PVC } \\
\text { raw material, } \\
\text { plastics } \\
\text { extrusion, } \\
\text { copper raw } \\
\text { material, and } \\
\text { copper wire } \\
\text { drawing } \\
\text { processes, } \\
\text { based on } \\
\text { measure } \\
\text { weight of } \\
\text { sample wire } \\
\text { and insulation }\end{array}$ & & & & & & & & \\
\hline Other & $\begin{array}{l}\text { Insulated } \\
\text { Wire }\end{array}$ & $\begin{array}{l}\text { Silicone } \\
\text { Insulated } \\
\text { Wire }\end{array}$ & $\begin{array}{l}\text { Sum of } \\
\text { silicone } \\
\text { material, } \\
\text { copper raw } \\
\text { material, and } \\
\text { copper wire } \\
\text { drawing } \\
\text { processes, } \\
\text { based on } \\
\text { measure } \\
\text { weight of } \\
\text { sample wire } \\
\text { and insulation } \\
\end{array}$ & & & & & & & & \\
\hline Other & $\begin{array}{l}\text { Chemicals } \\
\text { and Minerals }\end{array}$ & Mercury & $\begin{array}{l}\text { Values from } \\
\text { SimaPro }\end{array}$ & $\begin{array}{l}\text { Analyzing } \\
1 \mathrm{~kg} \\
\text { 'Mercury, } \\
\text { liquid, at } \\
\text { plant/GLO S' }\end{array}$ & $\begin{array}{l}\text { IPCC } \\
2007 \\
\text { GWP } \\
100 a \\
\text { V1.23 }\end{array}$ & $\begin{array}{l}\text { Cumulative } \\
\text { Energy } \\
\text { Demand } \\
\text { V1.05 / } \\
\text { Cumulative } \\
\text { energy } \\
\text { demand }\end{array}$ & Inventory* & & & & \\
\hline Other & $\begin{array}{l}\text { Chemicals } \\
\text { and Minerals }\end{array}$ & $\begin{array}{l}\text { Tungsten } \\
\text { Wire }\end{array}$ & $\begin{array}{l}\text { Sum of } \\
\text { Tungsten raw } \\
\text { material and } \\
\text { copper wire } \\
\text { drawing } \\
\end{array}$ & & & & & & & & \\
\hline Other & $\begin{array}{l}\text { Chemicals } \\
\text { and Minerals }\end{array}$ & $\begin{array}{l}\text { Tungsten } \\
\text { Raw } \\
\text { Material }\end{array}$ & $\begin{array}{l}\text { Values from } \\
\text { SimaPro }\end{array}$ & $\begin{array}{l}\text { Analyzing } \\
1 \text { kg } \\
\text { 'Tungsten l' }\end{array}$ & $\begin{array}{l}\text { IPCC } \\
2007 \\
\text { GWP } \\
100 a \\
\text { V1.23 }\end{array}$ & $\begin{array}{l}\text { Cumulative } \\
\text { Energy } \\
\text { Demand } \\
\text { V1.05 / } \\
\text { Cumulative } \\
\text { energy } \\
\text { demand }\end{array}$ & & & & & \\
\hline
\end{tabular}




\begin{tabular}{|c|c|c|c|c|c|c|c|c|c|c|c|}
\hline \multirow[b]{2}{*}{$\begin{array}{l}\text { Material } \\
\text { Class }\end{array}$} & \multirow[b]{2}{*}{ Material } & \multirow[b]{2}{*}{$\begin{array}{l}\text { Process } \\
\text { Name }\end{array}$} & \multirow[b]{2}{*}{$\begin{array}{l}\text { Description } \\
\text { of Data } \\
\text { Source }\end{array}$} & \multicolumn{4}{|c|}{ SimaPro } & \multicolumn{4}{|c|}{ GaBi } \\
\hline & & & & Title & $\begin{array}{l}\text { Method } \\
\text { - GHG }\end{array}$ & $\begin{array}{l}\text { Method - } \\
\text { Energy }\end{array}$ & $\begin{array}{l}\text { Method - } \\
\text { Mercury }\end{array}$ & Title & $\begin{array}{l}\text { Method - } \\
\text { GHG }\end{array}$ & $\begin{array}{l}\text { Method - } \\
\text { Energy }\end{array}$ & $\begin{array}{l}\text { Method - } \\
\text { Mercury }\end{array}$ \\
\hline Other & $\begin{array}{l}\text { Chemicals } \\
\text { and Minerals }\end{array}$ & Argon Gas & $\begin{array}{l}\text { Average of } \\
\text { values from } \\
\text { SimPro and } \\
\mathrm{GaBi}\end{array}$ & $\begin{array}{l}\text { Analyzing } \\
1 \mathrm{~kg} \text { 'Argon, } \\
\text { crude, liquid, } \\
\text { at plant/ } \\
\text { RER S' }\end{array}$ & $\begin{array}{l}\text { IPCC } \\
2007 \\
\text { GWP } \\
100 a \\
\text { V1.23 }\end{array}$ & $\begin{array}{l}\text { Cumulative } \\
\text { Energy } \\
\text { Demand } \\
\text { V1.05 / } \\
\text { Cumulative } \\
\text { energy } \\
\text { demand }\end{array}$ & Inventory* & $\begin{array}{l}\text { DE: Argon } \\
\text { (gaseous) } \\
\mathrm{PE}\end{array}$ & $\begin{array}{l}\text { CML2001, } \\
\text { Global } \\
\text { Warming } \\
\text { Potential } \\
\text { (GWP 100 } \\
\text { years) [kg } \\
\text { CO2-Equiv.] } \\
\text { kg CO2- } \\
\text { Equiv. }\end{array}$ & $\begin{array}{l}\text { Energy } \\
\text { renewable } \\
\text { and non- } \\
\text { renewable } \\
\text { (gross } \\
\text { calorific } \\
\text { value) [MJ] }\end{array}$ & Inventory* \\
\hline \multirow[t]{2}{*}{$\begin{array}{l}\text { Assembled } \\
\text { Components }\end{array}$} & $\begin{array}{l}\text { Edison } \\
\text { Screw Base } \\
\text { Assembly }\end{array}$ & $\begin{array}{l}\text { Steel } \\
\text { Edison } \\
\text { Base }\end{array}$ & $\begin{array}{l}\text { Sum of steel } \\
\text { sheet, steel } \\
\text { stamping, } \\
\text { and glass } \\
\text { products } \\
\text { processes, } \\
\text { based on } \\
\text { sample } \\
\text { components } \\
\end{array}$ & & & & & & & & \\
\hline & & $\begin{array}{l}\text { Glass } \\
\text { products }\end{array}$ & $\begin{array}{l}\text { Average of } \\
\text { values from } \\
\text { SimPro and } \\
\text { GaBi }\end{array}$ & $\begin{array}{l}\text { Analyzing } \\
1 \mathrm{~kg} \\
\text { 'Packaging } \\
\text { glass, white, } \\
\text { at plant/ } \\
\text { RER S' }\end{array}$ & $\begin{array}{l}\text { IPCC } \\
2007 \\
\text { GWP } \\
100 a \\
\text { V1.23 }\end{array}$ & $\begin{array}{l}\text { Cumulative } \\
\text { Energy } \\
\text { Demand } \\
\text { V1.05 / } \\
\text { Cumulative } \\
\text { energy } \\
\text { demand }\end{array}$ & Inventory* & $\begin{array}{l}\text { CH: Glass } \\
\text { (white; } \\
\text { packaging) } \\
\text { BUWAL }\end{array}$ & $\begin{array}{l}\text { CML2001, } \\
\text { Global } \\
\text { Warming } \\
\text { Potential } \\
\text { (GWP 100 } \\
\text { years) [kg } \\
\text { CO2-Equiv.] } \\
\text { kg CO2- } \\
\text { Equiv. }\end{array}$ & $\begin{array}{l}\text { Energy } \\
\text { renewable } \\
\text { and non- } \\
\text { renewable } \\
\text { (gross } \\
\text { calorific } \\
\text { value) [MJ] }\end{array}$ & Inventory* \\
\hline $\begin{array}{l}\text { Assembled } \\
\text { Components }\end{array}$ & $\begin{array}{l}\text { Edison } \\
\text { Screw Base } \\
\text { Assembly }\end{array}$ & $\begin{array}{l}\text { Brass } \\
\text { Edison } \\
\text { Base }\end{array}$ & $\begin{array}{l}\text { Sum of brass } \\
\text { raw material, } \\
\text { brass sheet } \\
\text { rolling, } \\
\text { aluminum } \\
\text { drawing, and } \\
\text { glass } \\
\text { products } \\
\text { processes, } \\
\text { based on } \\
\text { sample } \\
\text { components }\end{array}$ & & & & & & & & \\
\hline
\end{tabular}

\begin{tabular}{|c|c|c|c|c|c|c|c|c|c|c|c|}
\hline Energy & Energy Use & $\begin{array}{l}\text { US Grid } \\
\text { Mix }\end{array}$ & $\begin{array}{l}\text { Average of } \\
\text { values from } \\
\text { SimPro and } \\
\mathrm{GaBi}\end{array}$ & $\begin{array}{l}\text { Analyzing } \\
1 \mathrm{MJ} \\
\text { 'Electricity, } \\
\text { production } \\
\text { mix US/US } \\
\mathrm{S}^{\prime}\end{array}$ & $\begin{array}{l}\text { IPCC } \\
2007 \\
\text { GWP } \\
100 a \\
\text { V1.23 }\end{array}$ & $\begin{array}{l}\text { Cumulative } \\
\text { Energy } \\
\text { Demand } \\
\text { V1.05 / } \\
\text { Cumulative } \\
\text { energy } \\
\text { demand }\end{array}$ & Inventory* & $\begin{array}{l}\text { US: Power } \\
\text { grid mix PE }\end{array}$ & $\begin{array}{l}\text { CML2001, } \\
\text { Global } \\
\text { Warming } \\
\text { Potential } \\
\text { (GWP 100 } \\
\text { years) [kg } \\
\text { CO2-Equiv.] } \\
\text { kg CO2- } \\
\text { Equiv. }\end{array}$ & $\begin{array}{l}\text { Energy } \\
\text { renewable } \\
\text { and non- } \\
\text { renewable } \\
\text { (gross } \\
\text { calorific } \\
\text { value) [MJ] }\end{array}$ & Inventory* \\
\hline Energy & Energy Use & $\begin{array}{l}\text { EU Grid } \\
\text { Mix }\end{array}$ & $\begin{array}{l}\text { Average of } \\
\text { values from } \\
\text { SimPro and } \\
\mathrm{GaBi}\end{array}$ & $\begin{array}{l}\text { Analyzing } \\
1 \mathrm{MJ} \\
\text { 'Electricity, } \\
\text { production } \\
\text { mix } \\
\text { RER/RER S' }\end{array}$ & $\begin{array}{l}\text { IPCC } \\
2007 \\
\text { GWP } \\
100 a \\
\text { V1.24 }\end{array}$ & $\begin{array}{l}\text { Cumulative } \\
\text { Energy } \\
\text { Demand } \\
\text { V1.05 / } \\
\text { Cumulative } \\
\text { energy } \\
\text { demand }\end{array}$ & Inventory* & $\begin{array}{l}\text { EU-25: } \\
\text { Power grid } \\
\text { mix PE }\end{array}$ & $\begin{array}{l}\text { CML2001, } \\
\text { Global } \\
\text { Warming } \\
\text { Potential } \\
\text { (GWP 100 } \\
\text { years) [kg } \\
\text { CO2-Equiv.] } \\
\text { kg CO2- } \\
\text { Equiv. }\end{array}$ & $\begin{array}{l}\text { Energy } \\
\text { renewable } \\
\text { and non- } \\
\text { renewable } \\
\text { (gross } \\
\text { calorific } \\
\text { value) [MJ] }\end{array}$ & Inventory* \\
\hline
\end{tabular}

\begin{tabular}{|c|c|c|c|c|c|c|c|c|c|c|c|}
\hline End of Life & $\begin{array}{l}\text { Waste } \\
\text { Disposal }\end{array}$ & Landfill & $\begin{array}{l}\text { Average of } \\
\text { values from } \\
\text { SimPro and } \\
\mathrm{GaBi}\end{array}$ & $\begin{array}{l}\text { Analyzing } \\
1 \text { kg 'Landfill/ } \\
\mathrm{CHU} \text { ' }\end{array}$ & $\begin{array}{l}\text { IPCC } \\
2007 \\
\text { GWP } \\
100 a \\
\text { V1.23 }\end{array}$ & $\begin{array}{l}\text { Cumulative } \\
\text { Energy } \\
\text { Demand } \\
\text { V1.05 / } \\
\text { Cumulative } \\
\text { energy } \\
\text { demand }\end{array}$ & Inventory* & $\begin{array}{l}\text { RER: } \\
\text { Landfill } \\
\text { (Municipal } \\
\text { household } \\
\text { waste; BE, } \\
\text { DK) PE }\end{array}$ & $\begin{array}{l}\text { CML2001, } \\
\text { Global } \\
\text { Warming } \\
\text { Potential } \\
\text { (GWP 100 } \\
\text { years) [kg } \\
\text { CO2-Equiv.] } \\
\text { kg CO2- } \\
\text { Equiv. }\end{array}$ & $\begin{array}{l}\text { Energy } \\
\text { renewable } \\
\text { and non- } \\
\text { renewable } \\
\text { (gross } \\
\text { calorific } \\
\text { value) [MJ] }\end{array}$ & Inventory* \\
\hline
\end{tabular}




\begin{tabular}{|c|c|c|c|c|c|c|c|c|c|c|c|}
\hline \multirow[b]{2}{*}{$\begin{array}{l}\text { Material } \\
\text { Class }\end{array}$} & \multirow[b]{2}{*}{ Material } & \multirow[b]{2}{*}{$\begin{array}{l}\text { Process } \\
\text { Name }\end{array}$} & \multirow[b]{2}{*}{$\begin{array}{l}\text { Description } \\
\text { of Data } \\
\text { Source }\end{array}$} & \multicolumn{4}{|c|}{ SimaPro } & \multicolumn{4}{|c|}{$\mathrm{GaBi}$} \\
\hline & & & & Title & $\begin{array}{l}\text { Method } \\
\text { - GHG }\end{array}$ & $\begin{array}{l}\text { Method - } \\
\text { Energy }\end{array}$ & $\begin{array}{l}\text { Method - } \\
\text { Mercury }\end{array}$ & Title & $\begin{array}{l}\text { Method - } \\
\text { GHG }\end{array}$ & $\begin{array}{l}\text { Method - } \\
\text { Energy }\end{array}$ & $\begin{array}{l}\text { Method - } \\
\text { Mercury }\end{array}$ \\
\hline End of Life & $\begin{array}{l}\text { Waste } \\
\text { Disposal }\end{array}$ & Incineration & $\begin{array}{l}\text { Average of } \\
\text { values from } \\
\text { SimPro and } \\
\mathrm{GaBi}\end{array}$ & $\begin{array}{l}\text { Analyzing } \\
1 \mathrm{~kg} \\
\text { 'Incineration/ } \\
\mathrm{CH} \mathrm{U'}\end{array}$ & $\begin{array}{l}\text { IPCC } \\
2007 \\
\text { GWP } \\
100 a \\
\text { V1.24 }\end{array}$ & $\begin{array}{l}\text { Cumulative } \\
\text { Energy } \\
\text { Demand } \\
\text { V1.05 / } \\
\text { Cumulative } \\
\text { energy } \\
\text { demand }\end{array}$ & Inventory* & $\begin{array}{l}\text { RER: } \\
\text { Household } \\
\text { waste in } \\
\text { municipal } \\
\text { waste } \\
\text { incinerator } \\
\text { ELCD/PE- } \\
\mathrm{GaBi}\end{array}$ & $\begin{array}{l}\text { CML2001, } \\
\text { Global } \\
\text { Warming } \\
\text { Potential } \\
\text { (GWP 100 } \\
\text { years) [kg } \\
\text { CO2- } \\
\text { Equiv.] kg } \\
\text { CO2-- } \\
\text { Equiv. }\end{array}$ & $\begin{array}{l}\text { Energy } \\
\text { renewable } \\
\text { and non- } \\
\text { renewable } \\
\text { (gross } \\
\text { calorific } \\
\text { value) [MJ] }\end{array}$ & Inventory* \\
\hline End of Life & Reclamation & Recycled & $\begin{array}{l}\text { No values } \\
\text { included in } \\
\text { this release }\end{array}$ & & & & & & & & \\
\hline End of Life & Reclamation & $\begin{array}{l}\text { Remanu- } \\
\text { factured }\end{array}$ & $\begin{array}{l}\text { No values } \\
\text { included in } \\
\text { this release }\end{array}$ & & & & & & & & \\
\hline
\end{tabular}

* Inventory: Indicates that Mercury values were calculated from adding all sources of mercury identified in the output inventory of the associated process. See example below:

Values from $\mathrm{GaBi}$ (example is from Primary Aluminum Raw Material):

\begin{tabular}{|l|l|l|}
\hline $\begin{array}{l}\text { Mercury (+II) } \\
\text { [Heavy metals to } \\
\text { air] }\end{array}$ & $1.39394 \mathrm{E}-07$ & $\mathrm{~kg}$ \\
\hline $\begin{array}{l}\text { Mercury (+II) } \\
\text { [Heavy metals to } \\
\text { fresh water] }\end{array}$ & $1.19898 \mathrm{E}-08$ & $\mathrm{~kg}$ \\
\hline $\begin{array}{l}\text { Mercury (+II) } \\
\text { [Heavy metals to } \\
\text { sea water] }\end{array}$ & $1.5764 \mathrm{E}-09$ & $\mathrm{~kg}$ \\
\hline $\begin{array}{l}\text { Mercury (+II) } \\
\text { [Heavy metals to } \\
\text { industrial soil] }\end{array}$ & $3.00847 \mathrm{E}-12$ & $\mathrm{~kg}$ \\
\hline Sum & $1.52964 \mathrm{E}-07$ & $\mathrm{~kg}$ \\
\hline
\end{tabular}

Values from SimaPro (example is from Primary Aluminum Raw Material):

\begin{tabular}{|l|l|l|l|}
\multicolumn{2}{|l|}{ Material): } & $\begin{array}{l}\text { Aluminum, } \\
\text { primary, at } \\
\text { plant/RER S }\end{array}$ \\
\hline $\begin{array}{l}\text { Impact } \\
\text { category }\end{array}$ & Unit & Total & 0.4039905 \\
\hline Mercury & $\mathrm{mg}$ & 0.4039905 & \\
\hline
\end{tabular}

\footnotetext{
** Impact information for populated circuit boards in the LCA software tools was based on circuits used in the computing and telecommunications industries. Circuit boards in these industries tend to be very densely populated when compared to what was found in the LED products considered in this project, skewing the impact values for this category. In order to create a more representative set of impact values, the circuits were inventoried for five different lamps and the sums of the impacts of their circuit components were averaged per total mass of the populated circuit board assemblies. The values in the table above represent these averages.
} 



\title{
Appendix C - Sustainable LED Product Design Guide
}

The Sustainable LED Product Design Guide - a web-based tool is hosted by NCMS at www.ncms.org

\section{Sustainable LED Product Design Guide}

\author{
Available at www.ncms.org
}

User Manual

Rev 1

September 27, 2011

(j) ilumisys

\section{Overview}

The intent of the Sustainable LED Product Design Guide is to aid designers of LED lighting products in making environmentally sustainable design decisions. This guidance is provided through comparison against existing or conceptual designs through an interactive charting environment. In order to compare a design concept against other products, the user follows this procedure:

- Create the new product

- Identify the product type

- Populate the Bill-of-Materials (BOM) of the product

- Assign materials and processes to the components in the BOM

- Assign the performance specifications of the product

- Identify the disposal scenario expected for the product

- Proceed to the charting dashboard to compare the environmental impacts of the design against other products in the guide

- Export PDF charts in order to report findings 


\section{Creating or Reviewing a Product}
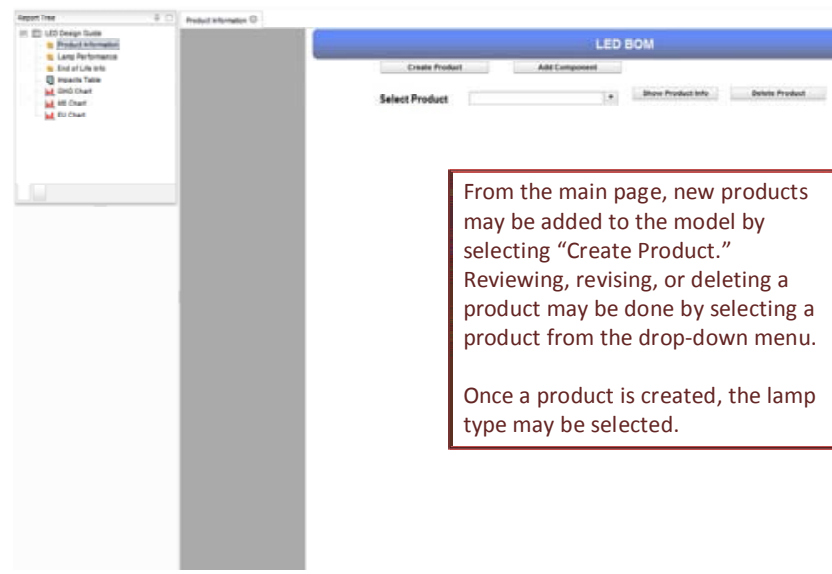

(i) ilumisys

Acins

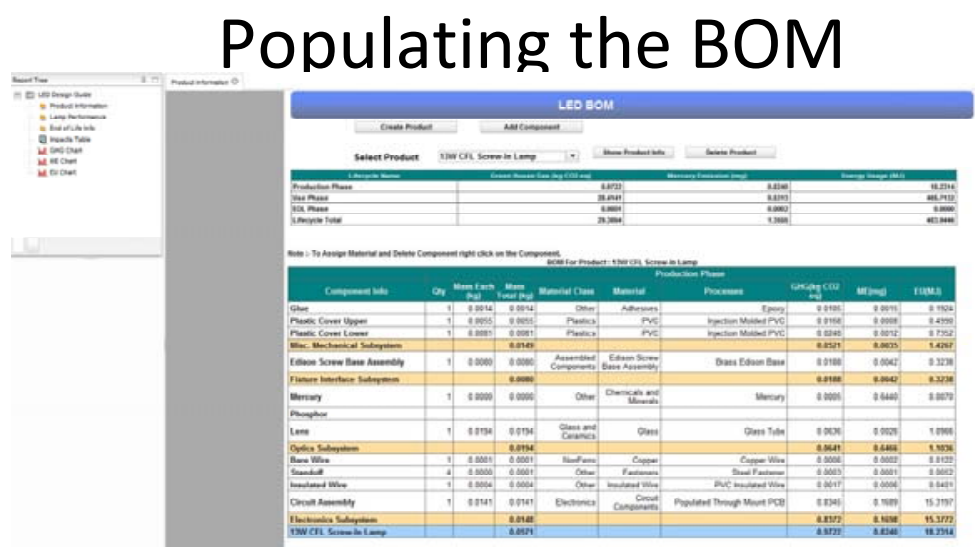

From the BOM page, components may be added and assigned to a subsystem. Once a component is added, materials and processes may be assigned by right-clicking the component and selecting "assign material."
(i) ilumisys
PCM 


\section{Assigning the Performance Specifications and End-of-Life Scenario to the Product}

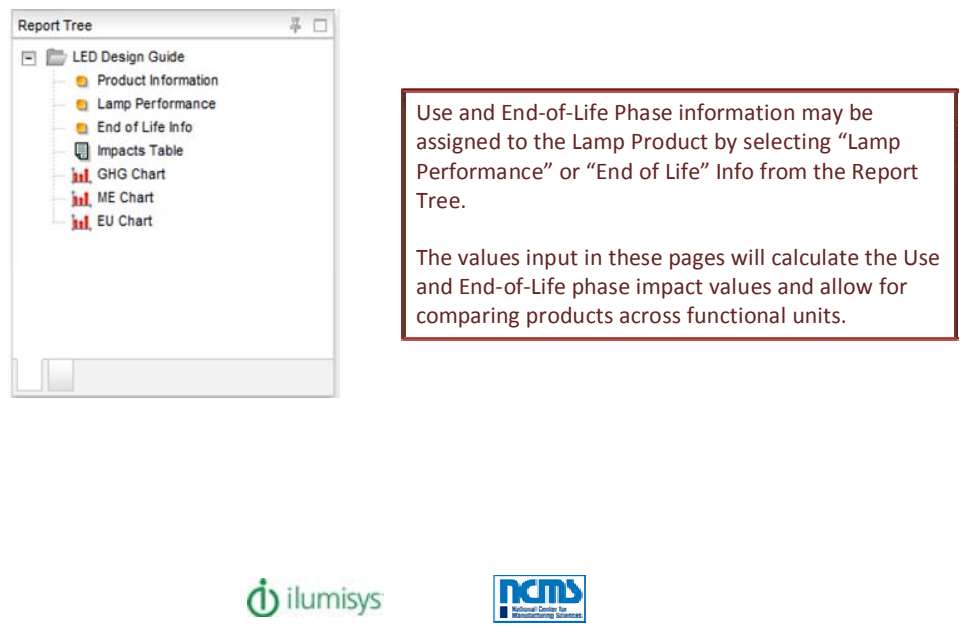

\section{Product Review Reports}

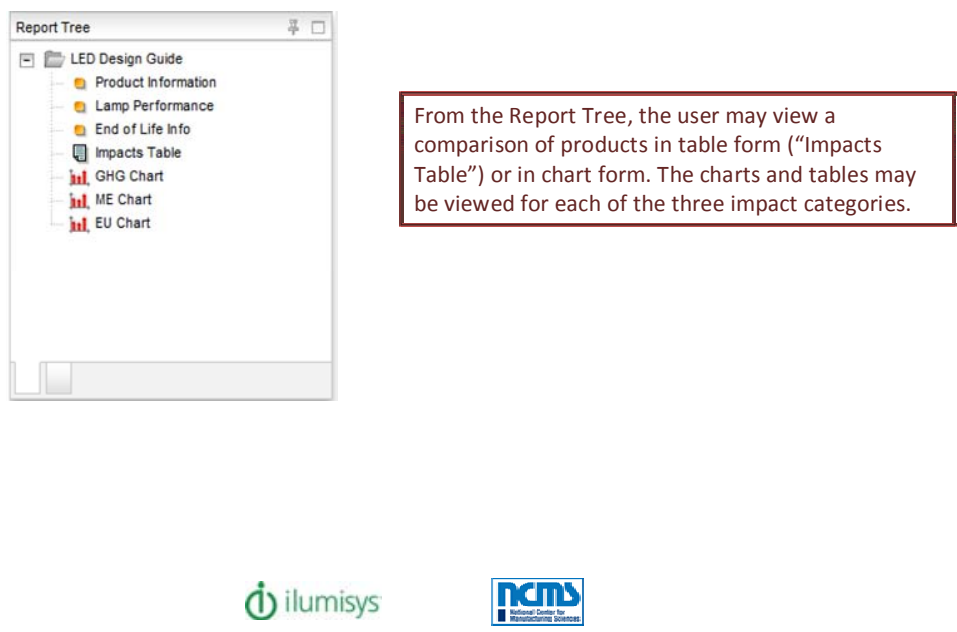




\section{Environmental Impacts Table}

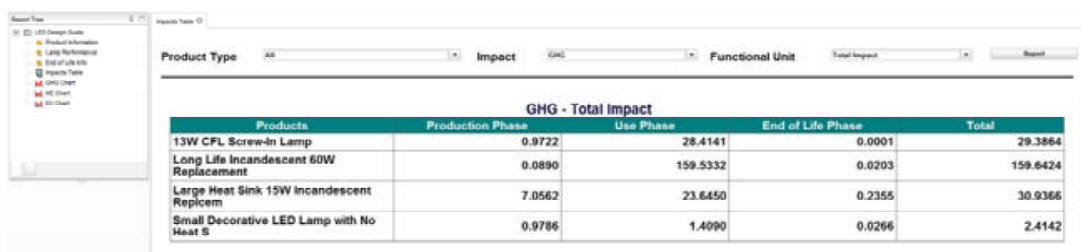

The Impacts Table shows a tabular comparison of

products' impacts.

The user may choose to show all of the products of a

given type, or all products in the "Product Type" field.

The user may select which impact category to compare

products against in the "Impacts" field.

The user may choose to compare the impacts of the products for total lifecycle impacts, impacts per hour of use, or impacts per lumen-hour. The normalization factor may be selected in the "Functional Unit" field.

(i) ilumisys

\section{Environmental Impacts Charts}

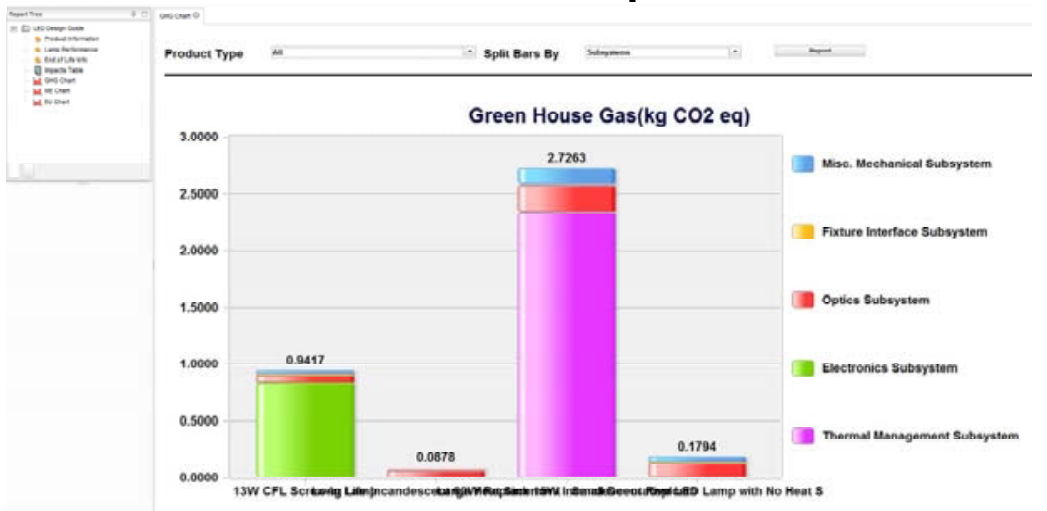

By choosing one of the impact category charts from the Report Tree, a visual report of the impacts of the various products is generated.

The bars may be divided by the subsystems for a comparison of Production Phase impacts, or by life cycle

phase for a comparison of products across life cycle phases with the option of selecting a functional unit.
(i) ilumisys
HCIs 


\section{Exporting Reports to PDF Files}

The icons at the top of the screen allow for exporting PDF files of the reports.

The first icon allows additional report pages to be created.

The second icon hides the Report Tree to maximize the viewing area for the charts and tables.

The third icon exports a PDF file of the currently active report page.

The fourth icon exports a PDF file for all of the tabs.

In order to create a report showing charts for all impact categories, new tabs would be created for each desired chart, and when complete, the fourth icon can be selected to export a report for the comparison of impacts.

(i) ilumisys





\section{Appendix D - Presentation Materials}

NCMS and Ilumisys presented the work done on this project at three different venues over the course of the project. The first was at the 2010 NeoCon conference in Chicago in June, 2010. The second was at the Southeast Michigan Sustainable Business Forum monthly meeting in September of 2010. The third presentation was given at the Sustainable Electronics Initiative conference in Champaign, IL in March 2011. The slides shown in Champaign are included below:
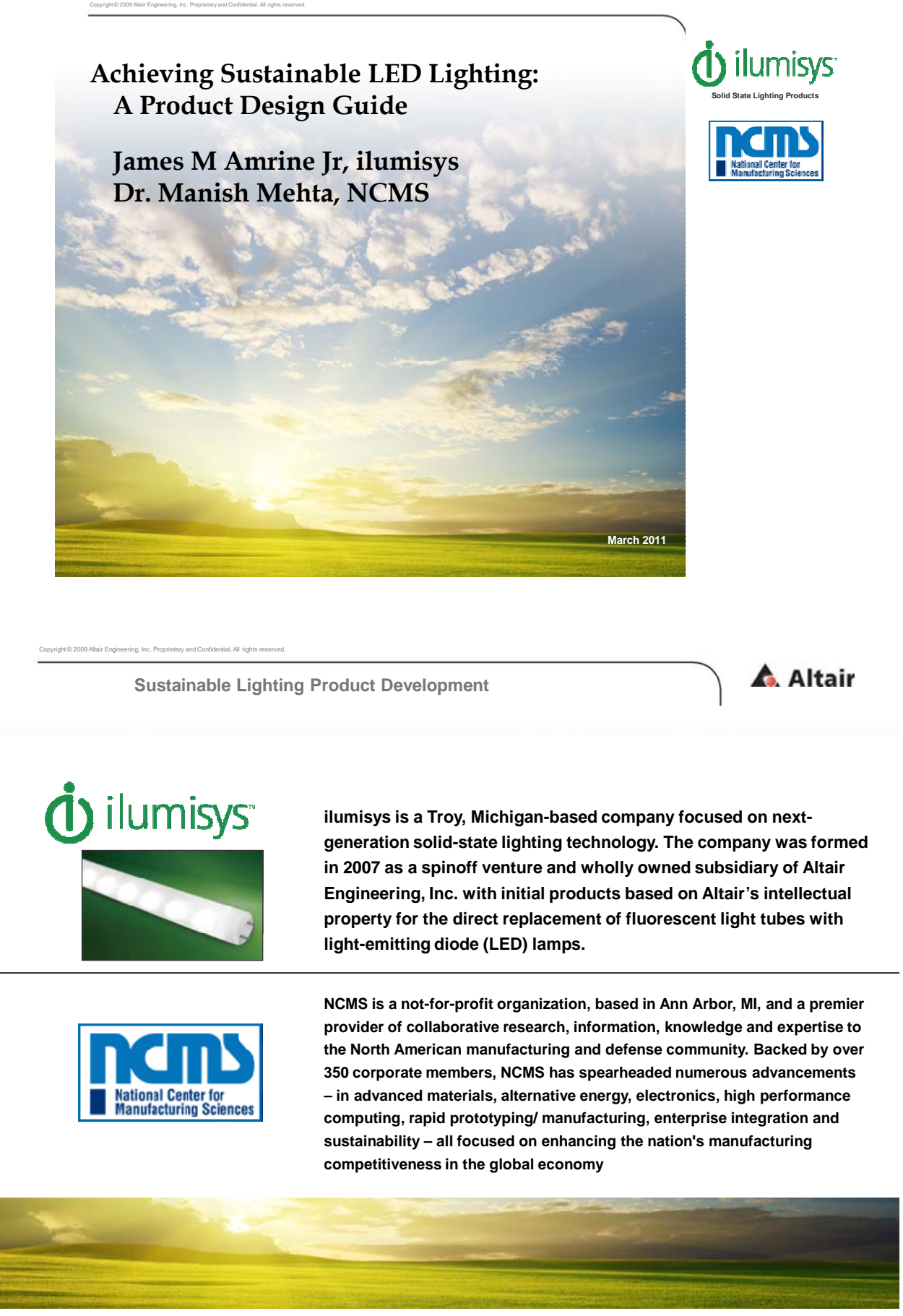


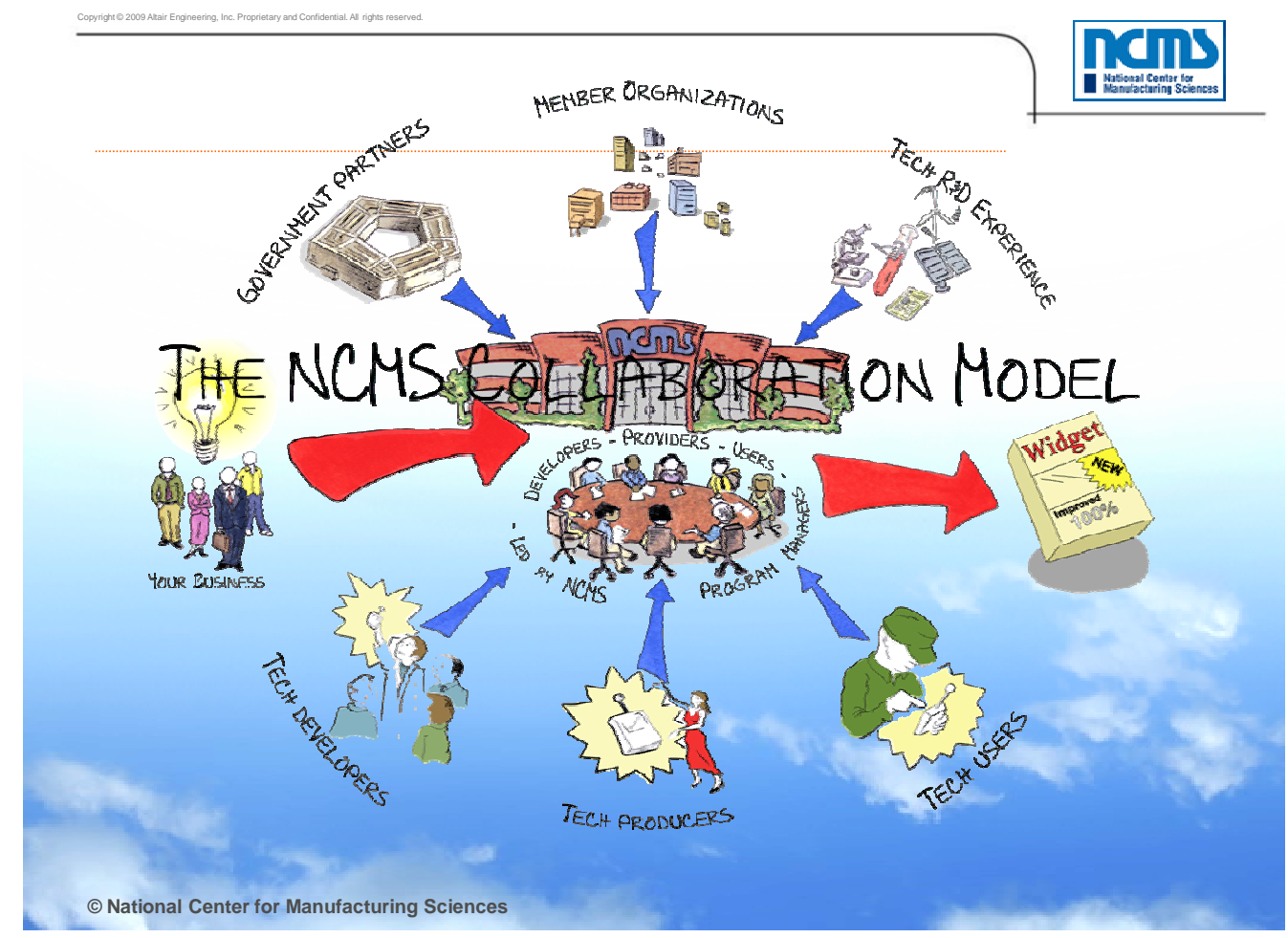

\section{NCMS Sustainability Program}

\section{1

- DOE Programs in Lightweighting, Fuel Cells \& Hydrogen

- DOE - Sustainable Light Emitting Diode Fluorescent Lamps (with ilumisys/Altair) - LCA-driven Design Guide/Web tool

- Operates 9 (of 14) EPA Compliance Assistance Centers (Web)

- EPA-LCA for VOCs in Paints \& Coatings Web tool

- $\quad$ EPA - Sustainable Product Initiative

1. Cradle-to-Gate Tools \& Gate-to-Gate tools

2. Remanufacturing Assessment Web tool

3. Sustainable Automotive Materials Selection Guide tool

4. National Green Vehicle Standard

5. Multi-Attribute Sustainability Standards Guide (Web) 


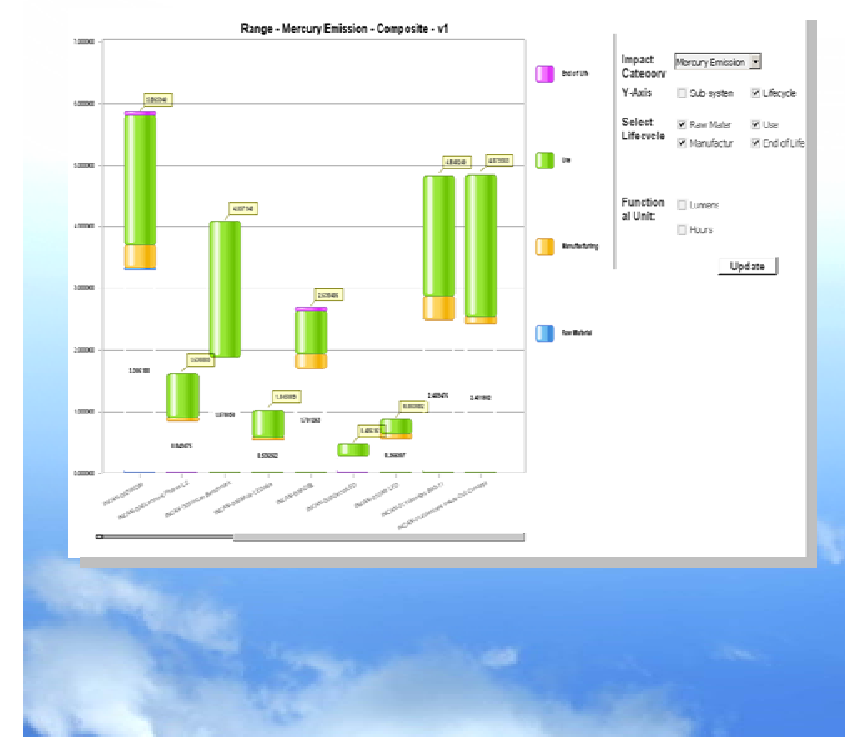

\author{
U.S. Department \\ of Energy sustainability \\ of LED lighting products \\ project
}

The U.S. Department of Energy (DOE) has contracted ilumisys and the National Center for Manufacturing Science (NCMS) to evaluate the sustainability of LED lighting products. ilumisys is conducting durability and output testing of numerous LED lighting products to define $a$ an evaluation process (including LCA).

The study findings will generate a best practices guide to allow LED product designers to make sustainable design decisions.

\title{
Phase 1: As-Is Analysis
}

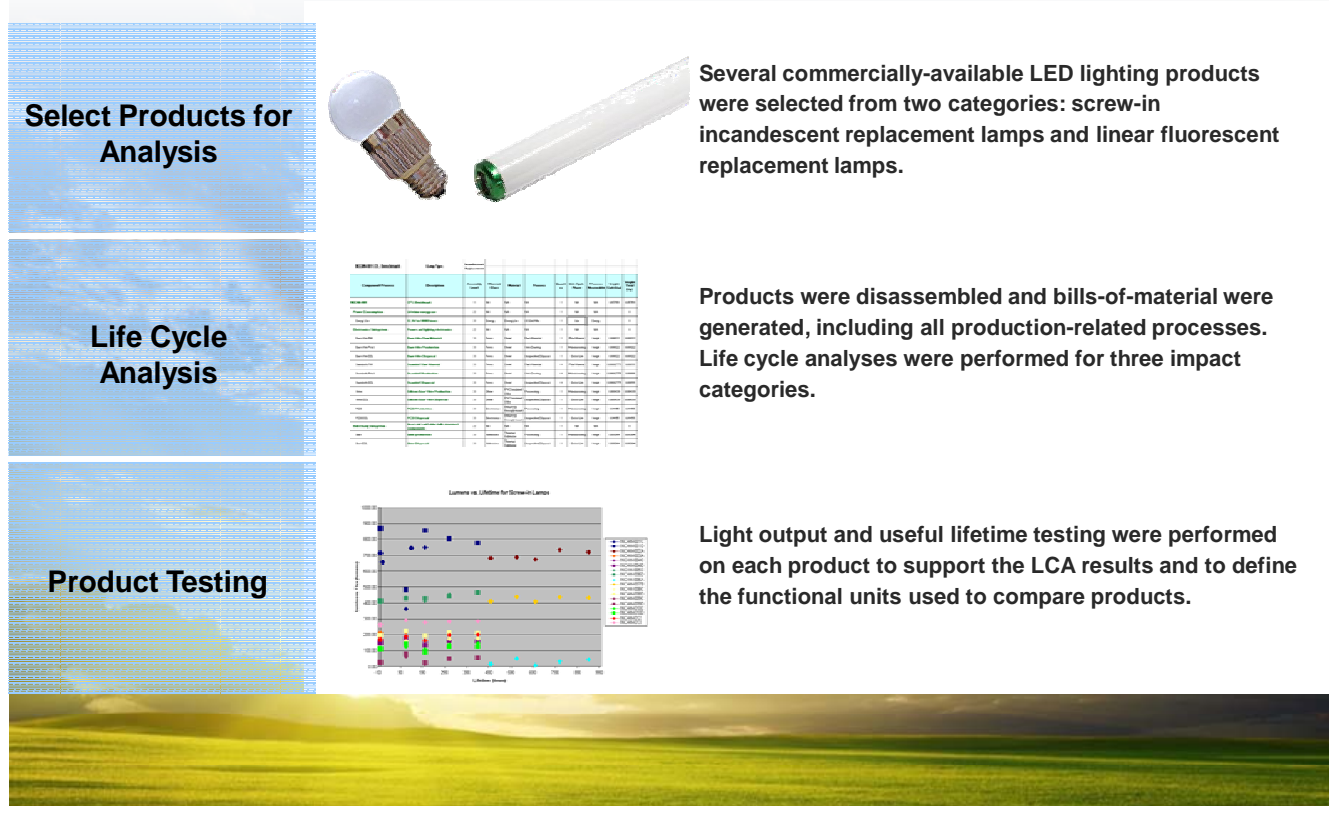



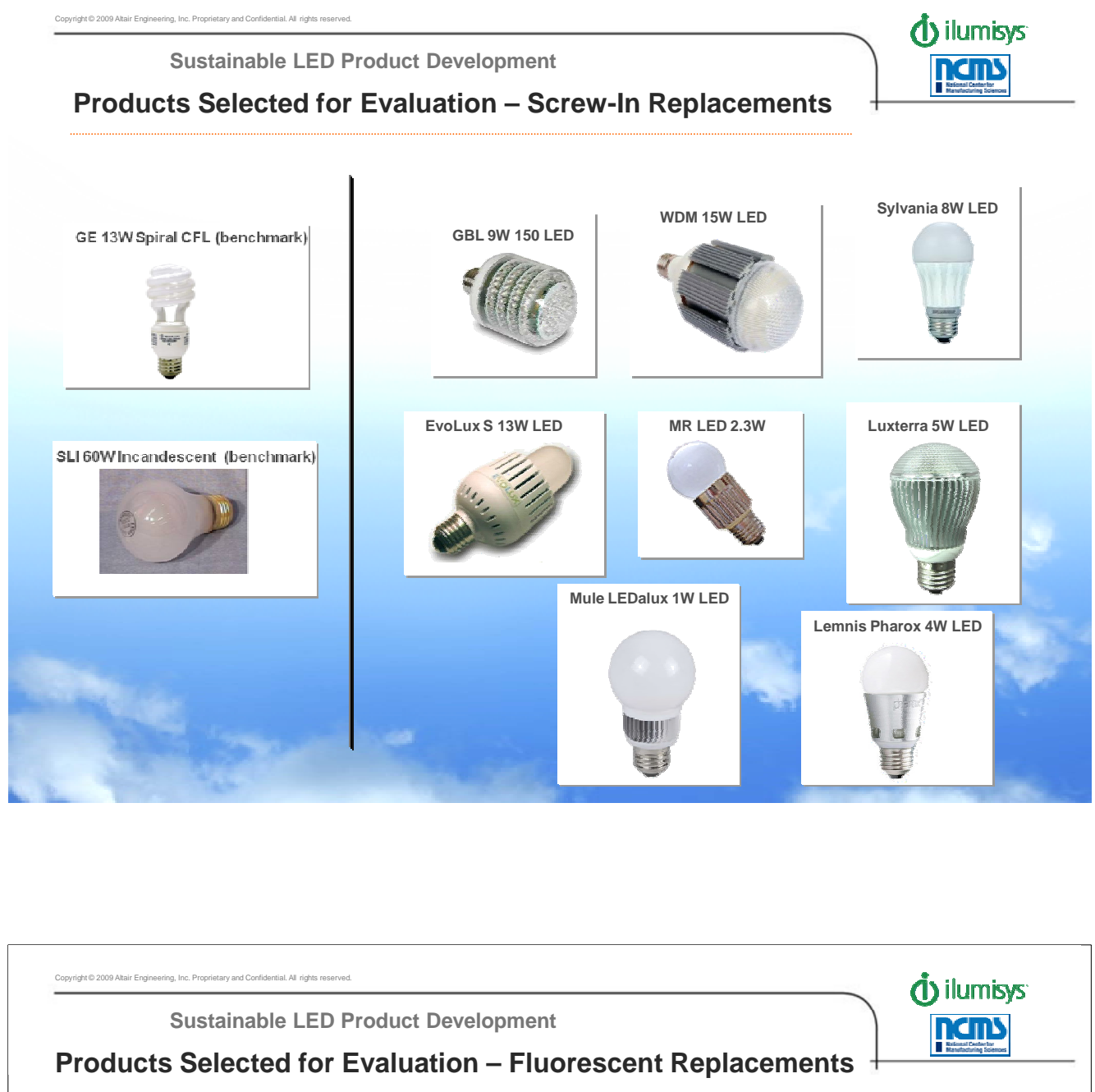

Products Selected for Evaluation - Fluorescent Replacements

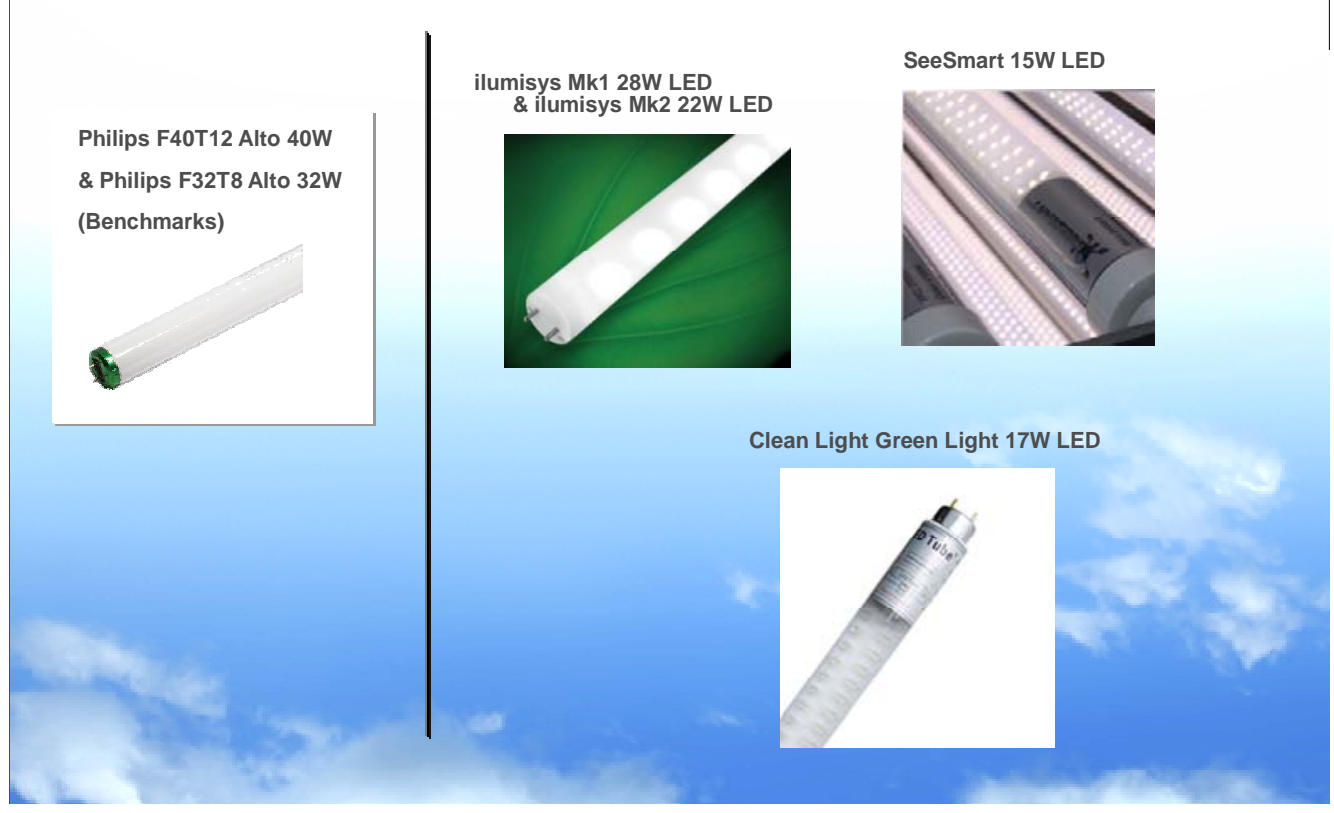




Sustainable LED Product Development
What is Life Cycle Assessment?
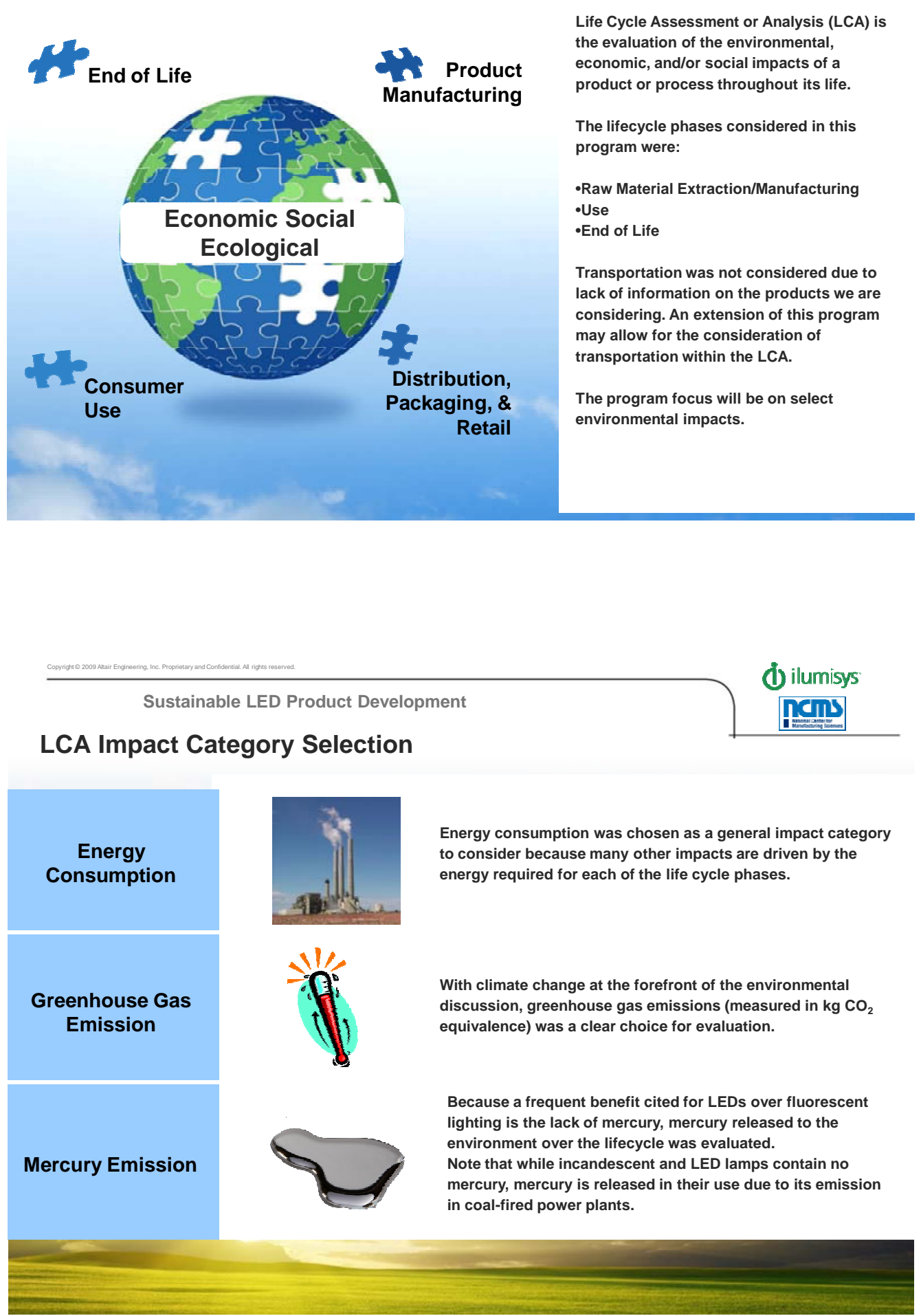


\section{Assumptions in the LCA Study}

\section{¿ilumisys}

- Populated circuit boards were considered by weight as a unit rather than as each of the constituent components. This is driving higher-than-realistic impacts for the electronics package. We are developing our own metric from the LCA software tools and measurements on actual lamp circuits to replace these values. This high impact per mass is due to the LCA inventories using telecom circuits and PC motherboards as their standard.

- "Unspecified" categories were established for use of primary, secondary, and production mix metals. Worst case of these defines the value for the unspecified condition.

- Default assumption for metals was production mix, as discussions with metals industry experts indicated that there is very little primary metal used except in very specific applications. The same is true of $100 \%$ secondary materials.

- Waste in any forming process was left to the defaults in the LCA software tools. Not enough information was available to identify waste, therefore only the mass of the finished part was used for impact calculations.

- Lamps that required a standard external electronic ballast (fluorescent tubes and the ilumisys Mk1 tubes) had the ballast impacts included in their LCA. The contribution of the ballast was based on a rated life of 50,000 hours and the assumption that a ballast runs two lamps, so if a ballast runs lamps that have a life of 25,000 hours, $1 / 4$ of the ballast impacts are included for a single lamp.

- Initially, useful life of the lamps, used in defining functional units, will be based on manufacturer claims. Lab testing will measure real life numbers and these will replace the other values as they become available.

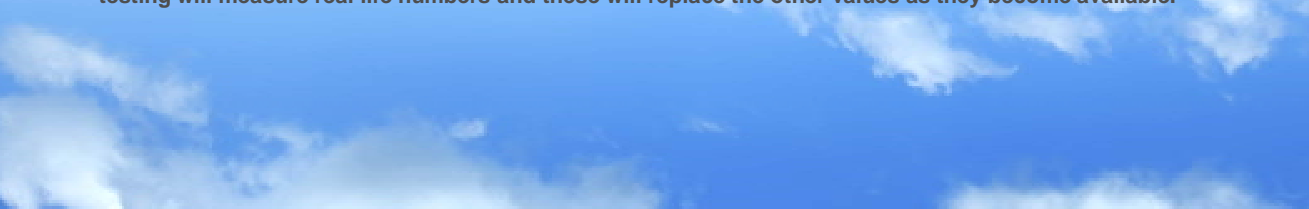

\section{Product BOM Creation}

\section{(j) ilumisys}

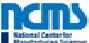

\section{Turning a bill-of-materials into a bill-of-processes...}

No longer only a 65-gram heat sink, now it is...

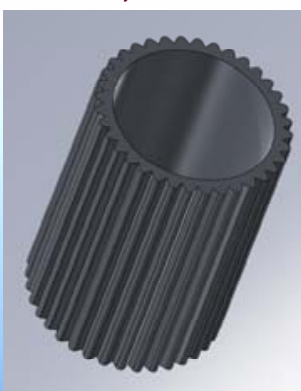

GHG Emissions from production and end-of-life phases: $\sim 1.12 \mathrm{~kg} \mathrm{CO}_{2}$ eq.

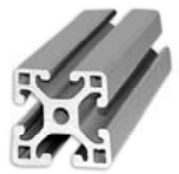

Extruding $\sim .06 \mathrm{~kg} \mathrm{CO}_{2} \mathrm{eq}$.

Anodizing $\sim .27 \mathrm{~kg} \mathrm{CO}{ }_{2}$ eq
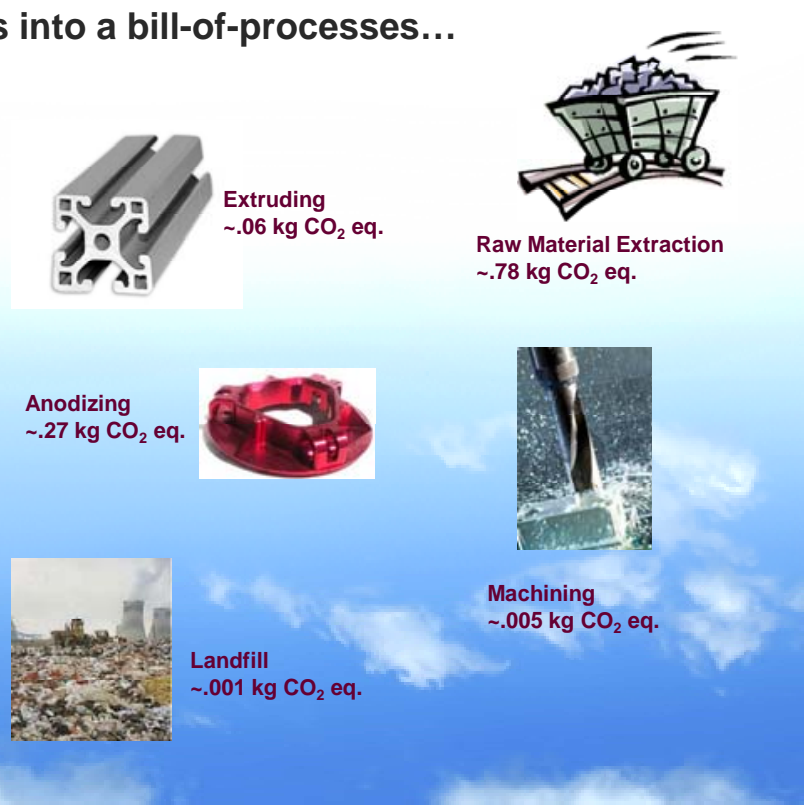


\section{Product Testing}

\section{¿ilumisys}

What is being tested, and why?

- Power consumption and useful life are needed to quantify the use phase in the life cycles of the lamps.

- Light output and useful life contribute to the functional unit against which products can be compared.

- Testing conditions are guided by best practices taken from Energy Star and IES LM-79 and LM-80 standards.

- Color temperature and color rendering index are also being measured.

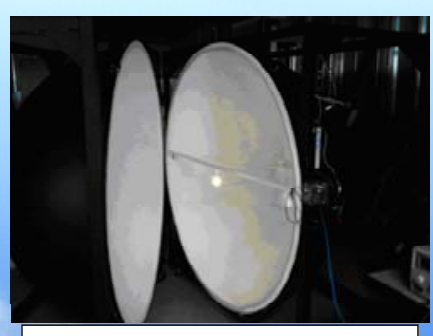

62 " integrating sphere for photometric testing

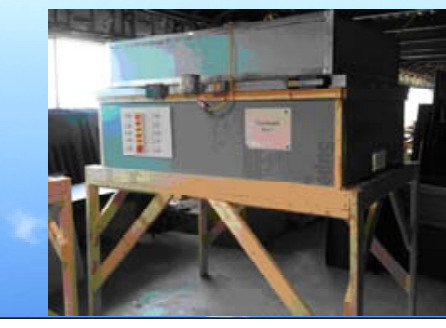

Lamps run continuously on a 3-hour on, 20minute off cycle at $45^{\circ} \mathrm{C}$ inside of temperature control chambers

\section{Some Preliminary Test Results - Lumens vs Time}

\section{(j) ilumisys}

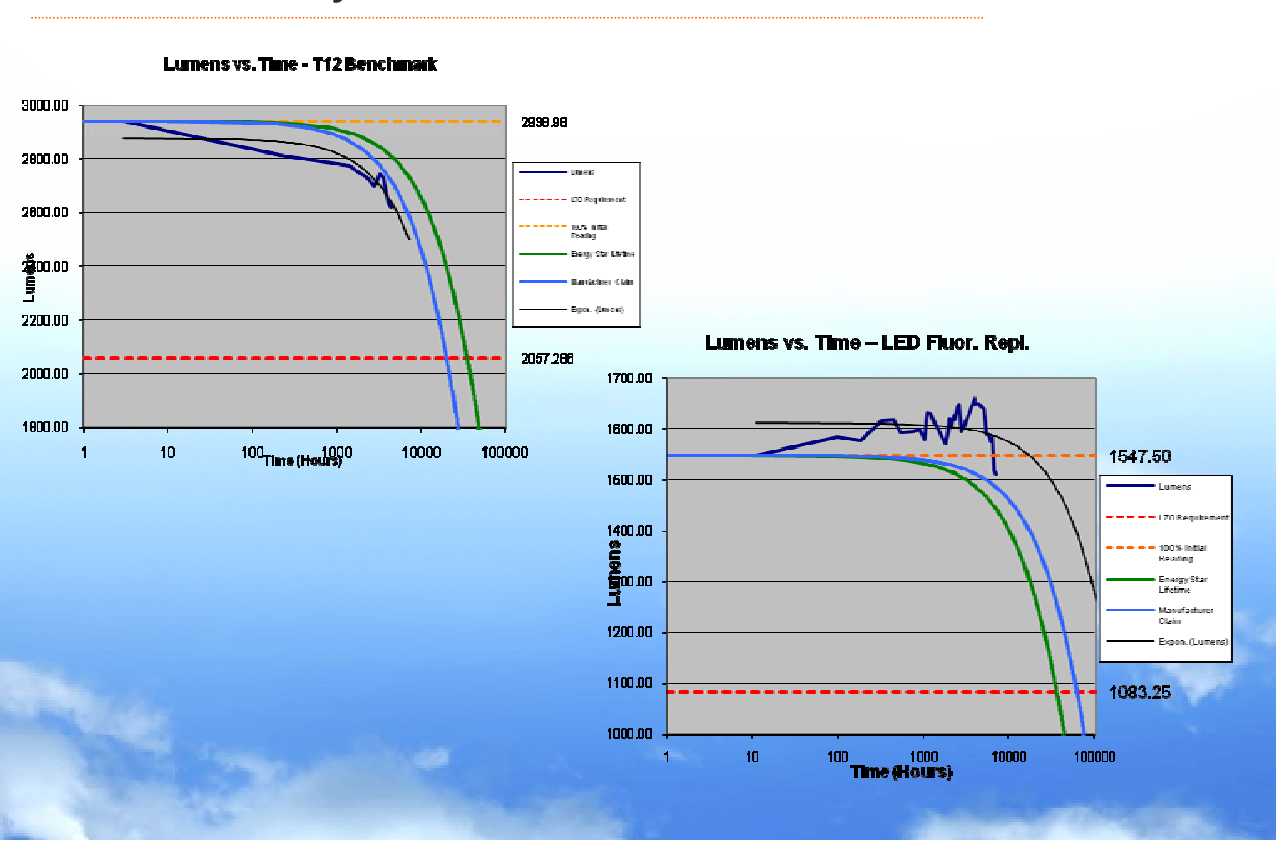




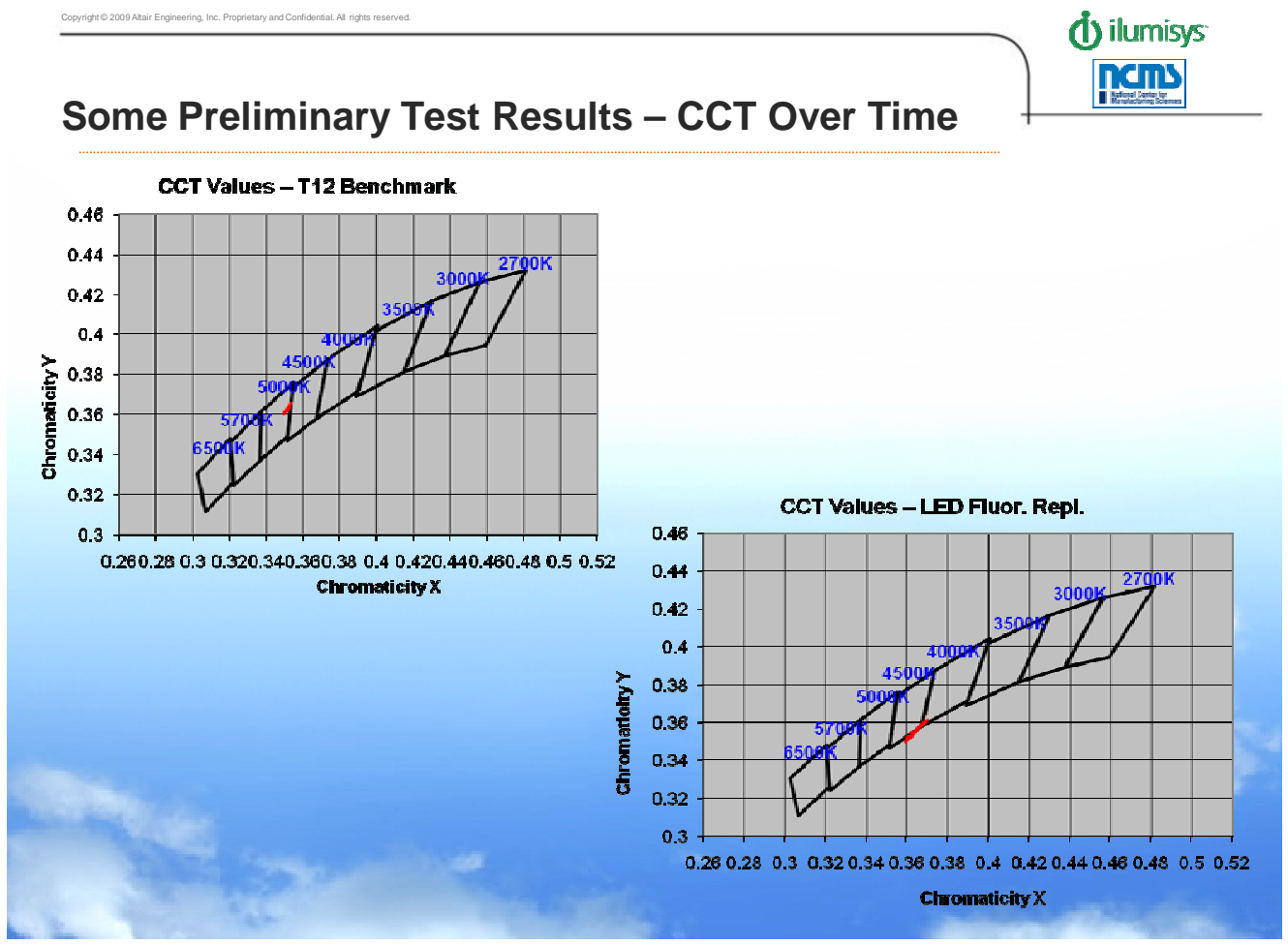

\section{Functional Units in LCA}

Why are functional units important?

Allow comparisons of products with different levels of performance without penalizing better performing products that have higher impacts

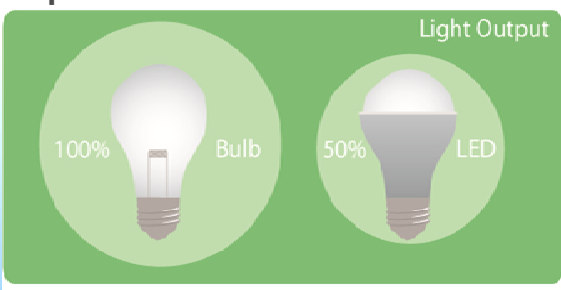

As an example, to get the same amount of light over the same period of use, it might require 15 incandescent lamps to equate to the performance over time of one LED lamp. Thus, in comparison, 15times the impacts of the

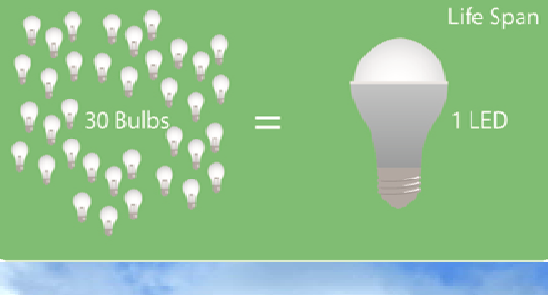
incandescent lamp are considered against a single LED lamp. 


\section{Functional Units in LCA}

\section{¿ilumisys}

When looking at single lamps, the LED lamps have very high impacts due to their complexity...
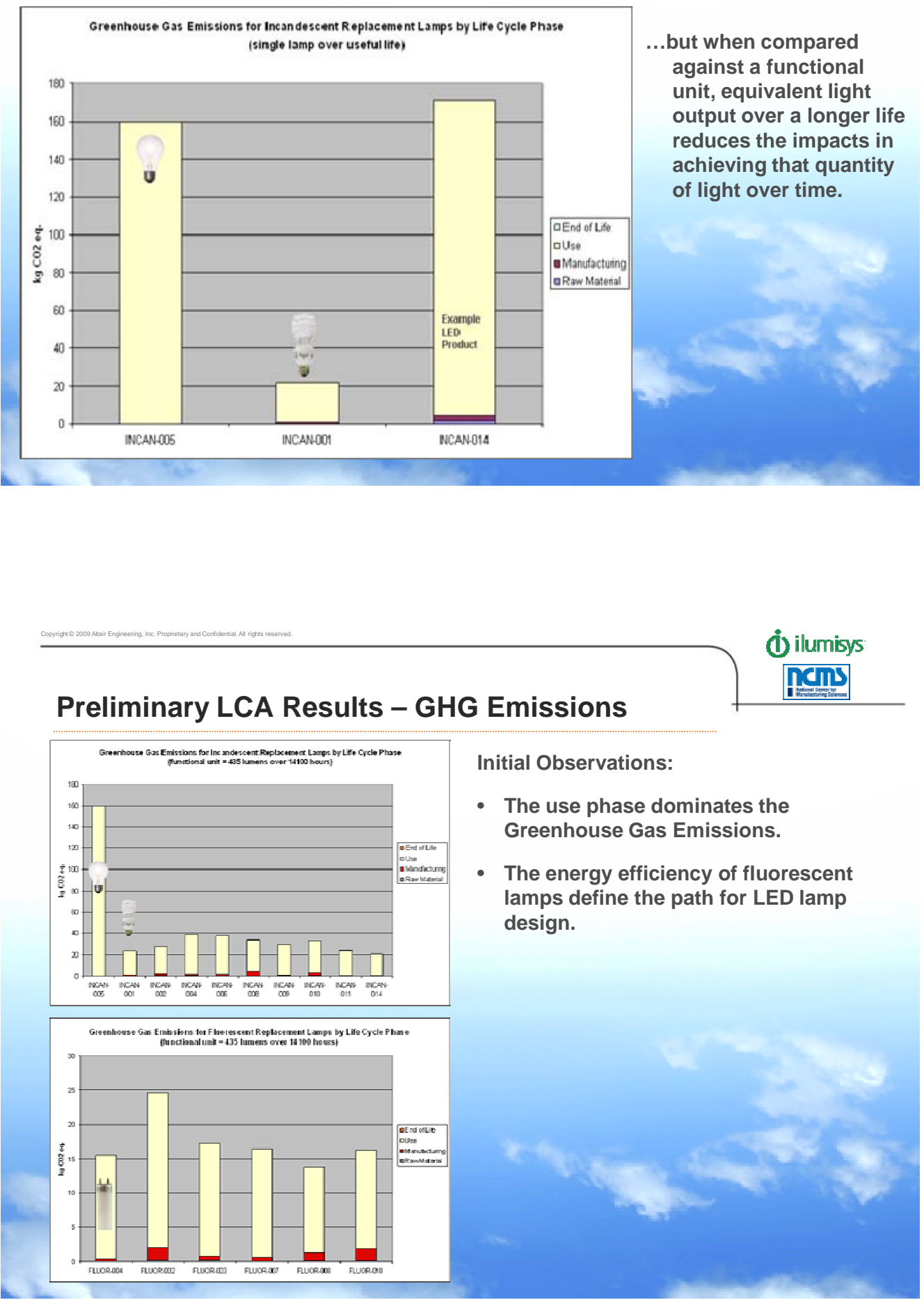


\section{Preliminary LCA Results - Energy Use}

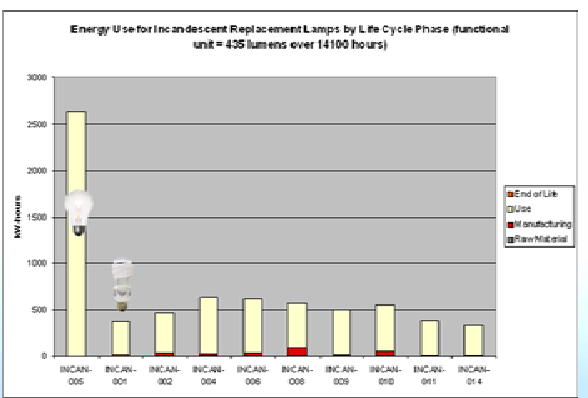

Initial Observations:

- The use phase dominates the Energy Use Impact.

- Energy Use tracks with GHG - GHG Emissions are directly related to Energy Use in all phases.

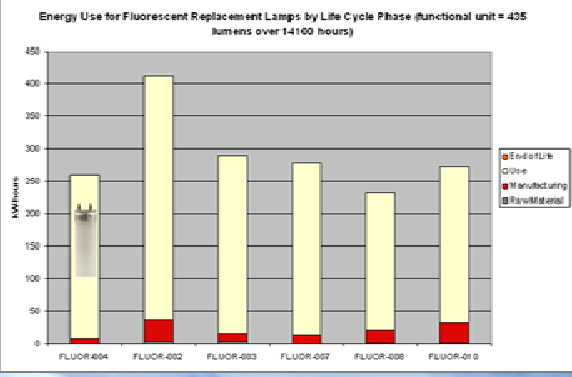

\section{(j) ilumisys}

\section{Preliminary LCA Results - Mercury Emissions}

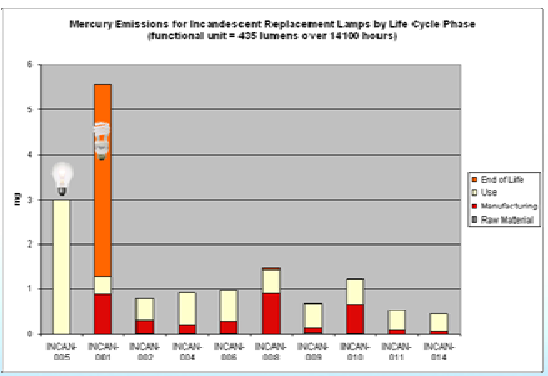

Initial Observations:

- The manufacturing stage plays a larger role in Mercury Emission than in other impact categories.

- The fluorescent products contain mercury which is accounted for at endof-life.

- Mercury emission comes primarily from coal-fired plants as incandescent and LED products contain no mercury.

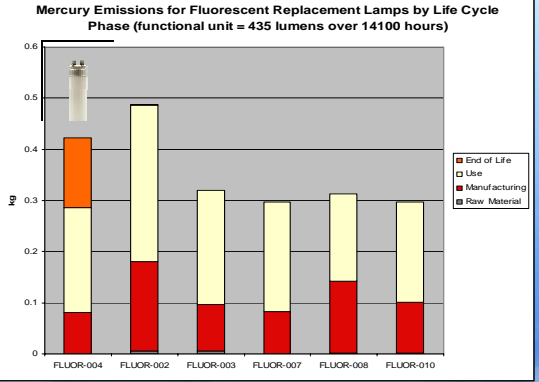

- Larger discrepancies are seen between data sources for Mercury Emission as compared to other impact categories. 


\section{Preliminary LCA Results - Without the Use Phase}

\section{¿) ilumisys}

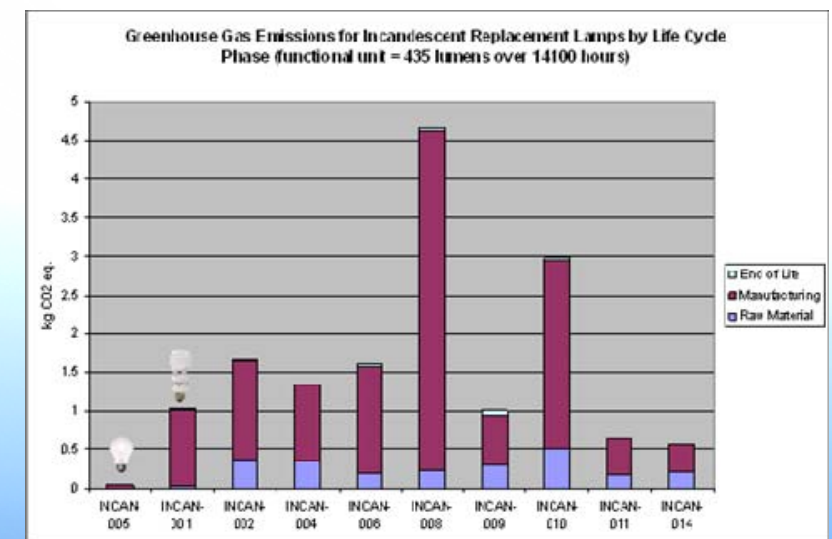

Initial Observations:

- Manufacturing is the phase with the second greatest potential for impact reductions, after improved efficacy of the lamp.

- The end-of-life phase remains as the phase with the lowest impacts.
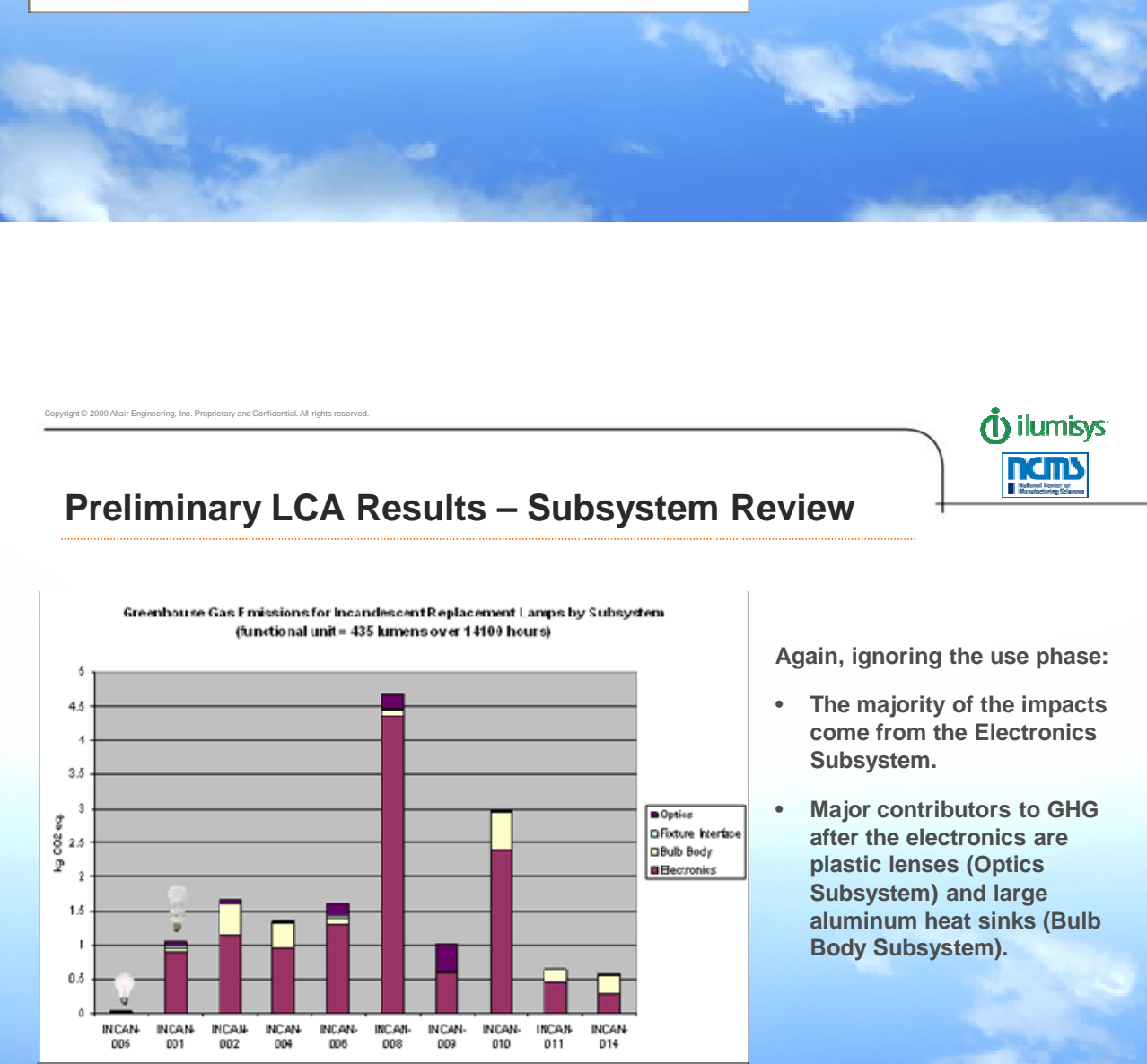

Again, ignoring the use phase:

- The majority of the impacts come from the Electronics Subsystem.

- Major contributors to GHG after the electronics are plastic lenses (Optics Subsystem) and large aluminum heat sinks (Bulb Body Subsystem). 

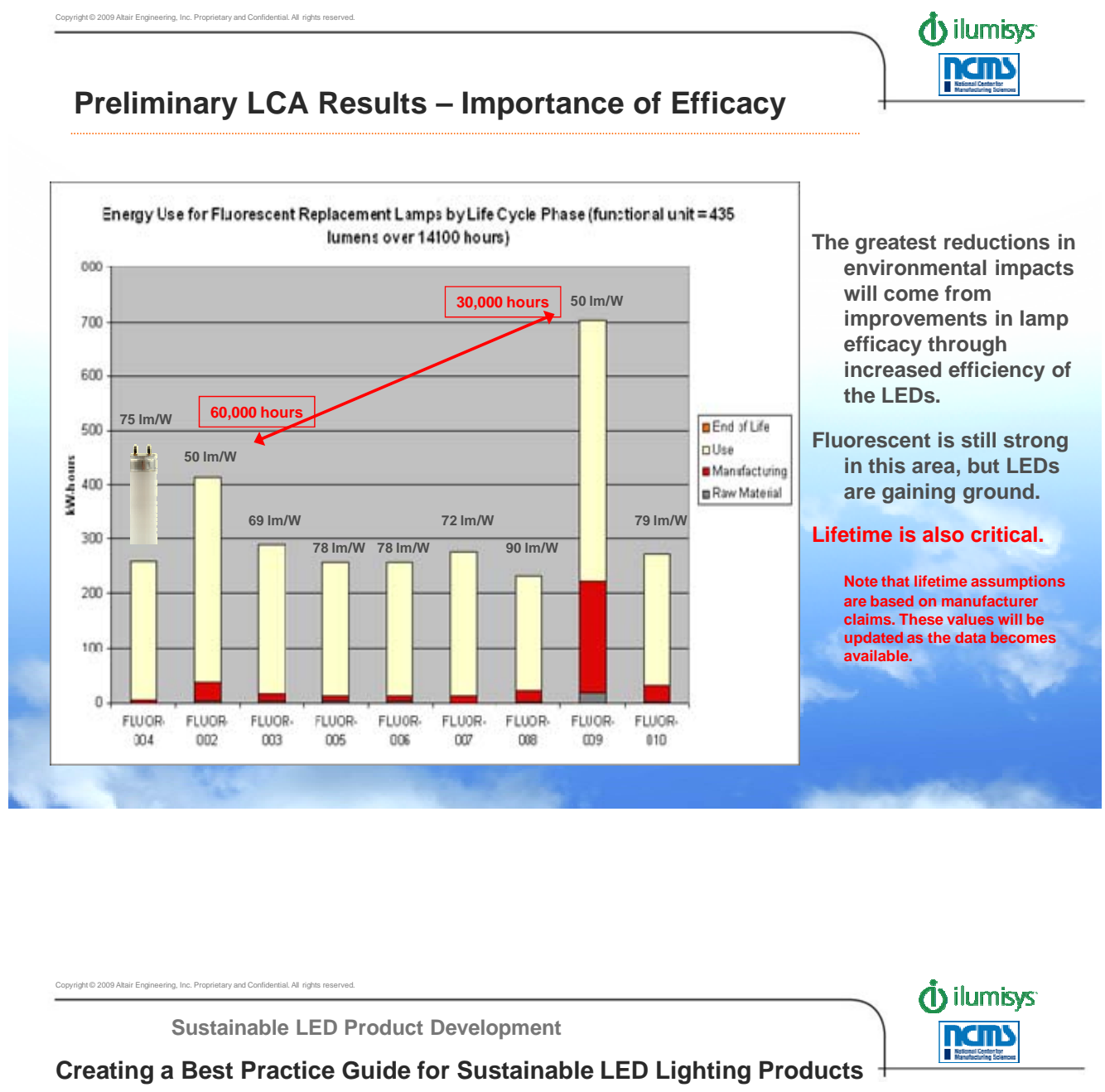

The design guide, when complete will be available via the sustainability portal on the NCMS website. The frame work of the tool will be as follows:

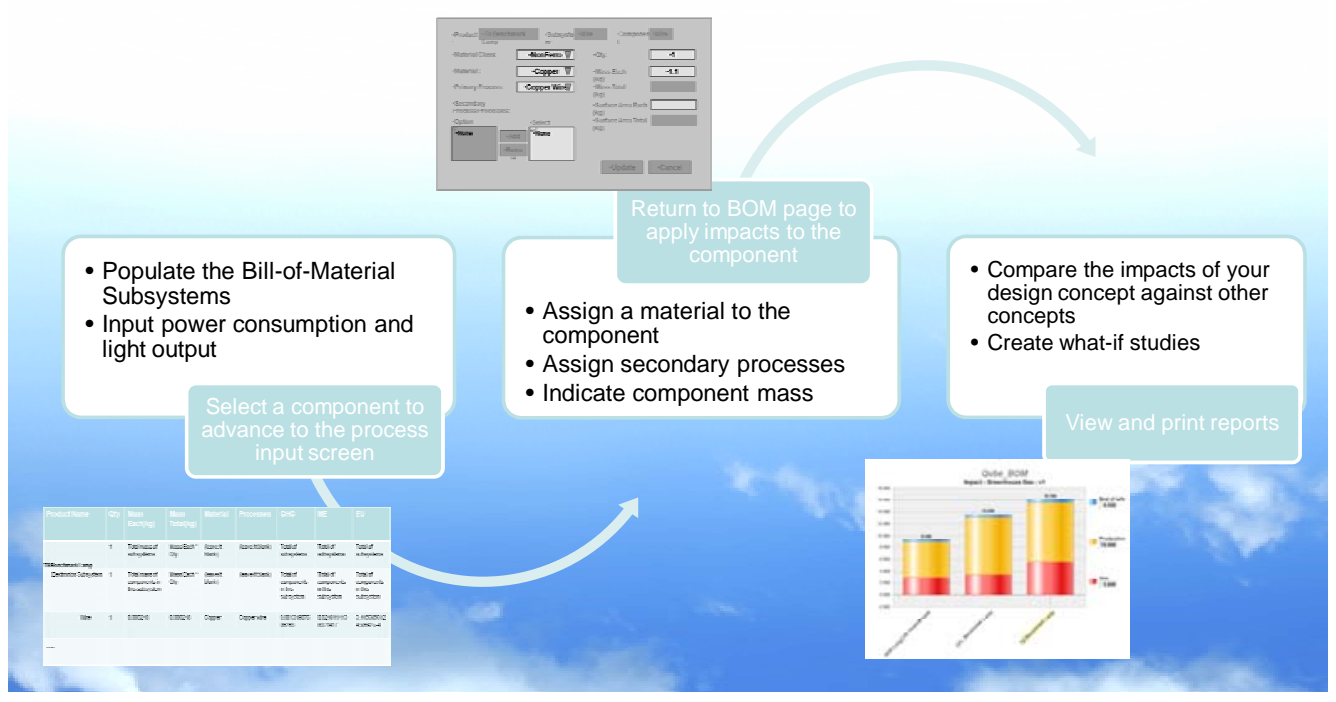

